\title{
139195010
}

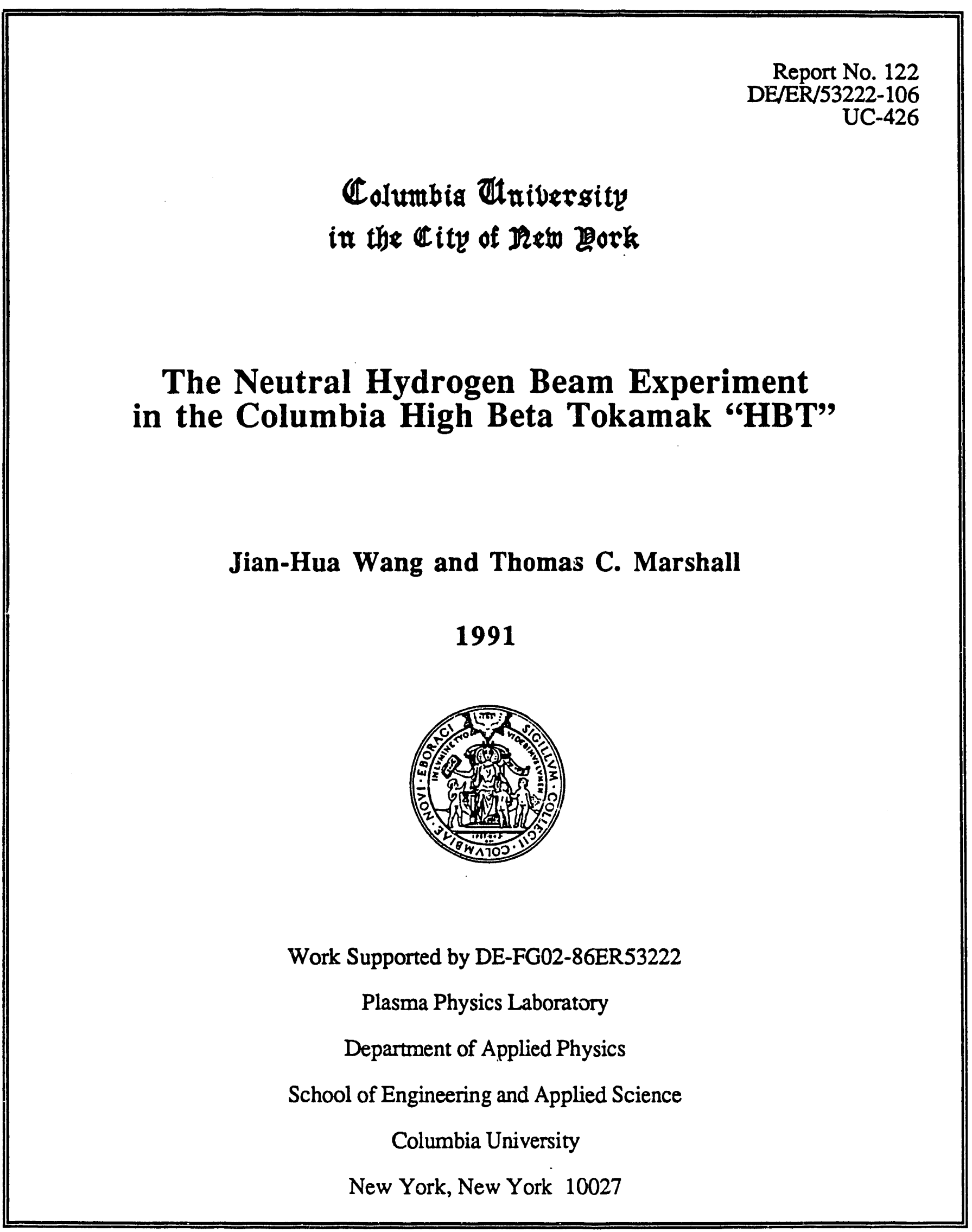




\section{The Neutral Hydrogen Beam Experiment in the Columbia High Beta Tokamak "HBT":}

\section{Part I: The Design of the Beam System Part II: The Orbit Simulation of Fast lons}

Jian-Hua Wang Thomas C. Marshall

Plasma Physics Laboratory

Columbia University 


\section{PART ONE: \\ THE DIAGNOSTICS OF NEUTRAL BEAM INJECTION IN THE COLUMBIA HIGH BETA TOKAMAK "HBT"}

\subsection{Introduction}

The neutral beam (NB) injection experiment was proposed as a new kind of diagnostic method for the Columbia HBT. We can use an injected neutral hydrogen beam (NHB) as a probe to detect certain properties of the HBT plasma. The energy of the NHB used in this experiment is $1 \mathrm{keV}$, which is higher than the thermal energy of the background plasma $\left(T_{e} \leq 25 \mathrm{eV}, n_{e} \leq 5 \times 10^{14} \mathrm{~cm}^{-3}\right)$. We could obtain some knowledge about the interaction of fast particles and tokamak plasma, for example, the fast particle confinement time, the energy transport from fast particles to plasma, etc. Such kind of information is very imprrtant to understand the physics of NB injection heating, which, it has been shown, is the most successful and hence the most popular technique of heating magnetically confined plasma to thermonuclear temperature. Also we can use the NHB as a way to examine certain MHD instabilities in HBT.

In part one of this report, the physics of NB injection is discussed, and then the NHB injector is described in detail. Finally, two methods of measurement in the 
experiment are introduced briefly.

\subsection{The Physics of NB Injection}

\subsubsection{The Trapping of Injected NB}

Once the NB is injected into HBT, the energetic neutrals must be ionized if these fast ions are to be confined long enough to share their surplus energy among the plasma particles. In general there are four atomic processes described as follows, which can ionize the incoming fast neutrals. They are:

(a) charge exchange

$$
\sigma_{c x}: \quad H^{(0)}+H_{p}^{(+)} \longrightarrow H^{(+)}+H_{p}^{(0)},
$$

where $H^{(0)}$ is the beam neutral, $H_{p}^{(+)}$the plasma ion and $\sigma_{c x}$ the cross section.

(b) plasma ion ionization

$$
\sigma_{i}: \quad H^{(0)}+H_{p}^{(+)} \longrightarrow H^{(+)}+H_{p}^{(+)}+e
$$

where $e$ is the electron, $\sigma_{i}$ is the ionization cross section by plasma ions.

(c) plasma electron ionization

$$
\sigma_{e}: \quad H^{(0)}+e \longrightarrow H^{(+)}+2 e,
$$

where $\sigma_{e}$ is the ionization cross section by plasma electrons.

(d) impurity ion ionization 
The ionization $\left(\sigma_{j i}\right)$ and charge exchanging $\left(\sigma_{j c x}\right)$ caused by all kinds of impurity ions.

Since the beam particles with velocity $v_{b}\left(v_{b}=4.4 \times 10^{7} \mathrm{~cm} / \mathrm{sec}\right.$ for $1.0 \mathrm{keV}$ NHB) are traveling much faster than the plasma ions $\left(v_{i}=2.5 \times 10^{6} \mathrm{~cm} / \mathrm{sec}\right.$ for helium plasma and $v_{i}=5.0 \times 10^{6} \mathrm{~cm} / \mathrm{sec}$ for hydrogen plasma at $T_{i}=25 \mathrm{eV}$, respectively), but much slower than the electrons $\left(v_{i}=2.1 \times 10^{8} \mathrm{~cm} / \mathrm{sec}\right.$ at $\left.T_{e}=25 \mathrm{eV}\right)$, then the effective cross section for trapping can be written approximately as:

$$
n_{e} \sigma_{e f f}=n_{i} \sigma_{c x}+n_{i} \sigma_{i}+\frac{n_{e}<\sigma_{e} v_{e}>}{v_{e}}+\sum_{j} n_{j}\left(\sigma_{j i}+\sigma_{j c x}\right),
$$

where $n_{e}, n_{i}$ and $n_{j}$ are the number densities of plasma electrons, plasma ions and the $j^{\text {th }}$ species of impurity ions, respectively; $\left\langle\sigma_{e} v_{e}\right\rangle$ indicates the average of rate coefficient of electron collisions over a Maxwellian distribution. The NB intensity $I(x)$ then can be expressed as :

$$
I(x)=I_{0} \exp \left\{-\int_{0}^{x} n_{e} \sigma_{e f f} d x\right\}
$$

where $x$ is taken along the beam direction. If $n_{e}$ and $n_{i}$ are constant and the impurity can be neglected, then we can get:

$$
I(x)=I_{0} \exp \left\{-n_{e} \sigma_{e f f} x\right\}=I_{0} \exp \left\{-\frac{x}{\lambda}\right\}
$$


where

$$
\lambda \equiv \frac{1}{n_{e} \sigma_{e f f}}
$$

is the mean free path for the ionization of the fast neutral particles. In fact, $n_{e}$ and $n_{i}$ are not constant, so the real energy deposition profile must be obtained by a more complicated calculation.

The useful cross section data for NB injection have been thoroughly discussed by Riviere in 1971, and some analytical expressions were formed which describe the collision data reasonably well. The data showed that when the beam energy is lower than $30 \mathrm{keV}$, charge exchange is the dominant process. More detailed calculation shows that for $1 \mathrm{keV} \mathrm{NHB,} \mathrm{the} \mathrm{cross} \mathrm{sections} \mathrm{of} \mathrm{different} \mathrm{atomic} \mathrm{processes,} \mathrm{for} \mathrm{the} \mathrm{hydrogen}$ plasma, are:

$$
\left\{\begin{array}{l}
\sigma_{i}=6.226 \times 10^{-19}\left(\mathrm{~cm}^{2}\right) \\
\sigma_{c x}=1.986 \times 10^{-15}\left(\mathrm{~cm}^{2}\right) \\
\frac{<\sigma_{e} v_{e}>}{v_{b}}=4.735 \times 10^{-17}\left(\mathrm{~cm}^{2}\right)
\end{array}\right.
$$

and, for the helium plasma, are:

$$
\left\{\begin{array}{l}
\sigma_{i}=6.979 \times 10^{-17}\left(\mathrm{~cm}^{2}\right) \\
\sigma_{c x}=1.862 \times 10^{-16}\left(\mathrm{~cm}^{2}\right) \\
\frac{<\sigma_{e} v_{e}>}{v_{b}}=4.735 \times 10^{-17}\left(\mathrm{~cm}^{2}\right),
\end{array}\right.
$$


respectively.

Since we are dealing with a bare nucleus for the helium plasma, the cross section should scale as $Z^{2}=4$ with respect to proton collision at an equal impact velocity (in the laboratory system). Under the assumption that $n_{e}=n_{i}=n_{0}=$ constant and neglecting the impurity contributions, we can get the effective cross section, $\sigma_{\text {eff }}$, for H-plasma:

$$
\sigma_{e f f}=2.034 \times 10^{-15}\left(\mathrm{~cm}^{2}\right)
$$

and for He-plasma is:

$$
\sigma_{e f f}=3.033 \times 10^{-16}\left(\mathrm{~cm}^{2}\right)
$$

where $\sigma_{e f f}$ is defined as:

$$
n_{e} \sigma_{e f f}=n_{e}\left[\sigma_{i}+\sigma_{c x}+\frac{<\sigma_{e} v_{e}>}{v_{b}}\right]
$$

The effective collision cross sections for $\mathrm{H}$ - and He-plasma are plotted in Fig. 1.1 and Fig.1.2 vs different beam energy, respectively. The mean free path for the ionization of fast neutrals in the HBT plasma (at the electron density $n_{e}=5.0 \times 10^{14} \mathrm{~cm}^{-3}$ ) is $\lambda=0.98 \mathrm{~cm}$ for H-plasma, and $\lambda=6.59 \mathrm{~cm}$ for He-plasma. Then the related effective mean collision time, $\tau_{e f f}$, defined as follows:

$$
\tau_{e f f} \equiv \frac{\lambda}{v_{b}}
$$


is $0.023 \mu \mathrm{sec}$ for $\mathrm{H}$-plasma, and $0.150 \mu \mathrm{sec}$ for He-plasma, respectively.

We have calculated the attenuation of NHB intensity for both constant and parabolic electron density profiles, see Fig.1.3 and Fig.1.4. It shows that the parabolic profile has some advantages for better penetration of the same $1 \mathrm{keV} \mathrm{NHB.}$

\subsubsection{The Orbits of Fast Ions in the HBT}

For a NB injection experiment, we need not only the adequate penetration of the beam, but also the well confined orbits in the magnetic structure of HBT. The fast ion (proton) trajectories depend on both the injection geometry with respect to the magnetic field and the total plasma current. The nonaxisymmetric magnetic field, the ripple field, which is due to the finite number and nonuniform winding of toroidal field coils, can also strongly affect the orbits of fast ions, especially at ripple level exceeding about $\pm 1-2 \%$.

Since the velocity of fast neutrals is much greater than the thermal velocity of plasma ions, an assumption of collisionless plasma has been used in the calculation of the fast protons orbits, and the results are described in the plotting of orbit projections onto both of the constant toroidal angle plane and the mid-plane (i.e. the vertical view at $Z=0$ ). The orbit simulation refer to three different cases, the simple tokamak mag- 
netic field, the simple tokamak magnetic field with constant ripple field modification, and the more realistic magnetic field for HBT from other simulations. The NHB is injected into the side port of the HBT vacuum vessel horizontally. For each case we considered two different injection geometries: co-injection (i.e. the toroidal component of the injection velocity is parallel to the plasma current) and counter-injection (i.e. the toroidal component of the injection velocity is anti-parallel to the plasma current). The NB injection geometry for HBT is partially tangential and partially perpendicular, with the injection angle (i.e. the angle between the beam and the direction which is perpendicular to the toroidal magnetic field) $\theta_{i}=14$ degrees. Our simulation results have shown several things as follows.

First of all, from the toroidal projection we can clearly see that there are two types of particle orbits: trapped and untrapped. The trapped particles mirror in the $B_{\phi}=\frac{B_{\phi 0}}{R}$ toroidal field and are confined near the outside of the plasma. The poloidal projection of the orbits clearly shows the banana shape orbits of the guiding center and the banana drift. However, the untrapped particles can circumnavigate the major and minor axes and the poloidal projection of their guiding center is shifted circle. Different ionization position (where the fast neutrals are ionized) results in a different type of orbit; for example, deeper penetration, for a simple tokamak, will produce 
more untrapped fast protons.

Secondly, under the condition of $15-20 k A$ plasma current, co-injection is much better than the counter-injection since the former yields more orbits that remain confined to plasma volume than the latter.

In third place, at the typical HBT operating parameters the fast protons resulting from the $1 \mathrm{keV}$ NHB can be well confined in the plasma for a reasonably long time

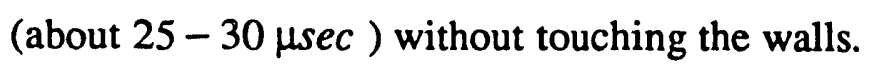

Finally, the toroidal field with $\pm 2 \%$ ripple modulation can still maintain good confinement for the fast protons. In fact the simulation result from the EFFI code shows that the ripple level for HBT is less than $1 \%$ on the axis of plasma (see Part Two).

We have made another orbit simulation for the case in which the $1(\mathrm{keV})$ NHB is injected vertically from the bottom port of the HBT vacuum vessel (because of the larger size compared with the side port). The results are also quite satisfactory for all of the features mentioned above. The second part of this report gives a detailed explanation of these orbit calculations. 


\subsubsection{The Thermalization of Fast Beam Ions}

The fast ions which are well confined inside the plasma transfer their energies to the plasma ions via a series of Coulomb collisions until they are thermalized. The average thermalization time $\tau_{t h}$, i.e. the time needed to slow the $1 \mathrm{keV}$ fast protons down to the plasma thermal energy, is given by the following equation:

$$
\tau_{t h}=\frac{\tau_{s}}{3} \ln \left[1+\left(\frac{E_{b}}{E_{c}}\right)\right]
$$

The thermalization time depends on not only the beam energy $E_{b}$, but also $E_{c}$, the critical energy at which the energy transferring rates, from the beam to either the protons and the electrons, are equal. $E_{c}$ is given by the following equation:

$$
E_{c}=1.2 \times \frac{Z_{b}^{4 / 3} m_{b} T_{e}}{m_{i}^{2 / 3} m_{e}^{1 / 3}} .
$$

In equation (1.10), $\tau_{s}$ is the Spitzer slowing-down time (i.e. the $90^{\circ}$ scattering time by multiple small angle Coulomb collisions) and is calculated as:

$$
\tau_{s}=6.27 \times 10^{8} \frac{A_{b}\left(k T_{e}\right)^{3 / 2}}{Z_{b}^{2} n_{e} \ln \Lambda}(\mathrm{sec})
$$

In the above equations, $A_{b}, Z_{b}$ and $m_{b}$ are the atomic number, charge and mass of the injected beam particles; $m_{i}$ and $m_{e}$ are the masses of plasma ions and electrons; $n_{e}$ is the plasma electron density in $\mathrm{cm}^{-3} ; T_{e}$ is the plasma electron temperature in $\mathrm{eV}$, 
respectively. In $\Lambda$ is the Coulomb logarithm which is approximately equal to 10 for the parameters of the HBT plasma.

For the Columbia HBT, typically at low to medium densities of helium or hydrogen plasmas with $\left\langle n_{i}\right\rangle=\left\langle n_{e}\right\rangle=5 \times 10^{14} \mathrm{~cm}^{-3}$, and $\left.\left\langle T_{i}\right\rangle=<T_{e}\right\rangle=25 \mathrm{eV}$, we find that under the condition of $E_{b}=1 \mathrm{keV}, Z_{b}=1$ and $A_{b}=1$, the three quantities, according to equation (1.10), (1.11) and (1.12), are:

$$
\left\{\begin{array}{l}
E_{c}=367.5(\mathrm{eV}) \\
\tau_{s}=15.7(\mu \mathrm{sec}) \\
\tau_{t h}=8.9(\mu \mathrm{sec})
\end{array}\right.
$$

for H-plasma, and are:

$$
\left\{\begin{array}{l}
E_{c}=146.5(\mathrm{eV}) \\
\tau_{s}=15.7(\mu \mathrm{sec}) \\
\tau_{t h}=15.4(\mu \mathrm{sec})
\end{array}\right.
$$

foe He-plasma, respectively.

In both cases, the thermalization time is shorter than the simulated fast proton orbit confinement time ( $-25 \mu \mathrm{sec})$, so the fast ions can well transfer their energy to plasma ions before touching the walls.

\subsection{The Technology of NHB Injector System}

The NHB is produced by neutralizing an energetic proton beam by passing it 
through a neutralizer which is filled with neutral hydrogen gas (see Fig. 1.5 for the general layout of the NHB system and Fig.1.6 for the vacuum system). The energetic beam is formed first at low energy by the ionization in a cold cathode discharge tube, and then extracted from the source plasma and accelerated in an electrostatic field. The proton beam is focused by a specially designed converging solenoid, inside which the accelerating grid and the discharge and neutralizer tubes are nested. The beam energy is $1 \mathrm{keV}$ and current (the flux) is about 1 Ampere with $100 \mu \mathrm{sec}$ pulse width, which can be adjusted from few to several hundreds micro-seconds.

\subsubsection{The Ion Generation}

A cold cathode discharge tube is used to produce the source plasma with low electron density (about $n_{i}=10^{10} \mathrm{~cm}^{-3}$ ) and low electron temperature (about $T_{e} \leq 0.5 \mathrm{eV}$ ), shown in Fig.1.7. The inner diameter of the tube is $10 \mathrm{~cm}$ and the diameter of the opening hole for the ion extraction surface (i.e. the disc) is $5 \mathrm{~cm}$. This means that the spatial variations of the source plasma can be neglected for the extraction disc. Hydrogen gas is fed into the tube through a specially designed puff-gas valve, shown in Fig.1.8, which is operated by the pulsed current in a RLC discharge circuitry. 


\subsubsection{The Ion Extraction}

A single stage, three electrode accel-decel-rating component is used to extract and accelerate the ions (protons) from the source plasma, shown in Fig.1.9(a). The small decel voltage well (about $200 \mathrm{~V}$ ), applied across the second gap, is used to prevent the electrons born in the neutralizer from going back to the source plasma region.

The saturation value of the ion flux which can be extracted from the source plasma is given by the following equation:

$$
J_{i}=n_{i} e\left(\frac{k T_{e}}{m_{i}}\right)
$$

For the plasma condition in the source region (i.e. inside the discharge tube) of $n_{i}=$ $10^{10} \mathrm{~cm}^{-3}, k T_{e}=1.2 \mathrm{keV}$ and $m_{i}=m_{p}=1.67 \times 10^{-24} \mathrm{~g}$, we can get the ion saturation current as:

$$
J_{i}=0.0543\left(A / \mathrm{cm}^{2}\right)
$$

The total ion current extracted from the source plasma is:

$$
I_{i}=J_{i} S_{d}=1.1(A)
$$

where $S_{d}$ is the area of the extraction surface (i.e. the disc). Thus the ion flux (hence the ion current $I_{i}$ ) is fixed by the operating conditions of the source plasma and the 
extracting surface area.

Considering the rate of change of ion momentum, the conservation of ion current and the adequate boundary conditions, we can get the ion current density, as the function of the voltage $V(l)$ and the distance $l$ of two successive electrodes on which the voltage applied, as follows:

$$
J_{i}=\frac{4 \varepsilon_{0}}{9\left[\frac{m_{p}}{2 e}\right]^{1 / 2}} \frac{V^{3 / 2}}{l^{2}}\left(A / m^{2}\right)
$$

If we choose $J_{i}=0.0543\left(A / \mathrm{cm}^{2}\right)$, then the distances of the accelerating $\left(d_{a}\right)$ and the decelerating $\left(d_{d}\right)$ gaps are:

$$
\left\{\begin{array}{l}
d_{a}=1.2(\mathrm{~mm}) \\
d_{d}=0.5(\mathrm{~mm})
\end{array}\right.
$$

The more accurate solution (Coupland, et al., 1973) showed that for a given extraction voltage $V$ applied across the extraction gap $d$, the extracted current $I$, passing through an aperture with radius $a$, is:

$$
\frac{I}{V^{3 / 2}}=1.72 \times 10^{-7}\left[\frac{Z}{A}\right]^{1 / 2}\left[\frac{a}{d}\right]^{2}\left[1-1.6\left(\frac{d}{r_{c}}\right)\right]
$$

where $A$ and $Z$ are the atomic and charge number of the beam ions respectively; $r_{c}$ is the radius of curvature of the emitting surface (i.e. the source plasma boundary). For a given extraction voltage and aperture radius, the current $I$ is proportional to $\left[\frac{1}{d^{2}}\right]$; 
so we should decrease the gap distance $d$ to increase the current. However, there is a limit to how small $d$ can be, set by the voltage breakdown. It also has been found that the divergence is a strong function of the aspect ratio $\left[\frac{a}{d}\right]$. The smaller the value of $\left[\frac{a}{d}\right]$ (usually $<1$ ), the lower the beam emittance. So we should reduce the value of $\left[\frac{a}{d}\right]$ by decreasing the aperture diameter to get better convergence of a single beamlet. Therefore, the multi-beamlet (i.e. to open more apertures on the extraction disc) structure is necessary to get more total current. In our design, $a=0.5 \mathrm{~mm}$ and $d=$ $1.2 \mathrm{~mm}$, so $\left[\frac{a}{d}\right]=0.42<1$ is a good value for the optical requirement to each beamlet (i.e. the beam from a single aperture). The different aperture shapes, as shown in Fig.1.9(b), also strongly influence the beamlet optical quality.

\subsubsection{The Species Yield of Ion Source}

The direct electric discharge in hydrogen can produce three species of ions: $H_{1}^{+}$, $H_{2}^{+}$and $H_{3}^{+}$. The extracted ion beam from this kind of source plasma generally consists of all of the three types of ions. But the composition (i.e. the fraction of each species) is usually difficult to predict from the operating parameters of the discharge process. Since all of three species of ions are singly charged, they all have the same 
energy after the acceleration by the biased grid, but different velocity because of the mass differences. After the neutralization, one $H_{1}^{+}$ion gives rise to one hydrogen atom carrying energy $E_{0}$, while one $H_{2}^{+}$ion gives rise to two hydrogen atoms each carrying $\frac{E_{0}}{2}$ energy, and one $H_{3}^{+}$ion gives rise to three hydrogen atoms each carrying $\frac{E_{0}}{3}$ energy. The lower energy species of neutrals, after injection into the plasma, are usually deposited at the outside of the plasma, which is undesirable. The different species will have different deposition profiles within the HBT plasma.

\subsubsection{The Neutralization of Fast Ions}

When the $1 \mathrm{keV} \mathrm{H}+$ ion beam passing through the neutralizer, it will be neutralized to become a neutral hydrogen atom beam primarily caused by charge exchange. Some data has shown that at the $1 \mathrm{keV}$ beam energy, the neutralization efficiency is as high as to about $90 \%$ (see Fig.1.10), and the cross section of charge exchange $\sigma_{c x}$ is about $4 \times 10^{-16} \mathrm{~cm}^{2}$. When the pressure of hydrogen gas inside the neutralizer is $2 m$ Torr, we can calculate the mean free path for an fast ion to become a fast atom as $\lambda=40 \mathrm{~cm}$. The length of the neutralizer was chosen as the same as $\lambda$ to get the highest efficiency of neutralization. The cross section of the inverse process, i.e. the reionization of fast neutrals caused by the neutralizer plasma is about $4 \times 10^{-17} \mathrm{~cm}^{2}$. So the 
probability of reionization is only $10 \%$ of the neutralization process and can be neglected.

\subsubsection{The Focusing of the Neutral Hydrogen Beam}

As we mentioned before that the multiple beamlets of fast protons extracted from the source plasma are mainly focused by a specially designed converging solenoid. The beam focusing angle, which is equal to the ratio of the radius of extraction surface to the focal length of the solenoid $(L=164 \mathrm{~cm})$, is about $0.87^{\circ}$. This is the maximum focusing angle, which is a function of the radial location in the solenoid and equal to zero when the solenoid axis approached. The principle of the design of the solenoid is the flux conservation of the magnetic field inside the solenoid which is produced by a pulsed current, as shown in Fig.1.11, since the transverse component of the B-field is fairly small near the longitudinal axis (because of the small focusing angle) and can be neglected.

The numerical calculation of A-contours and B-field strength along axis from the EFFI code is shown in Fig.1.12 for a uniformly wound solenoid, in Fig.1.13 for one uniformly and one non-uniformly wound solenoids and in Fig.1.14 for a different configuration of the converging solenoid from that as shown in Fig.1.13. 
Because of the converging structure of magnetic field inside the solenoid, it forms a magnetic mirror for the fast proton bean:. The general idea for a magnetic mirror is shown in Fig.1.15(a) and (b). We can make an estimate of the mirror effects. From the design and operating parameters we know that the "loss cone" angle of this mirror is about $31.1^{\circ}$, which is much larger than the beam focusing angle $0.87^{\circ}$, so all of the fast protons will be inside the loss cone and propagate without any trapping by t.e mirror. If a proton is still not neutralized after passing through the neutralizer, the transverse component of its velocity will increase a small percentage, about $3 \%$, which comes from the calculation considering the conservation of both the total kinetic energy and the magnetic moment. This is the worst case. This kind of proton will be swept away by a permanent magnet and can be neglected because of the high neutralization efficiency $(-90 \%)$ for a low energy $(1 \mathrm{keV})$ beam.

If we inject the beam vertically from the bottom of the HBT, we don't need to focus the beam since the bottom port has much larger diameter $(\sim 5.0 \mathrm{~cm})$ which allows a beam with larger cross section to pass through.

\subsubsection{The Emittance of the Neutral Hydrogen Beam}

The emittance effect of the NHB depends on the thermal velocity of the source plasma. In the presence of an axial magnetic field, when the temperature is not zero, 
the ions will move in helical trajectories about the field line and this results in a small spread (which corresponds to a small solid angle $d \Omega$ ) of the beam particle on the transverse plane. We can make an estimate as follows.

Suppose at the beginning of the discharge in the source region the plasma ions are cold (i.e. $T_{i}=0$ ), and the electrons are relative hot, say $T_{e}=3(e V)$, which is the typical electron temperature in a cold cathode discharge tube. Then an energy transportation will occur among electrons, ions and neutrals according to the following equations:

$$
\frac{d T_{i}}{d t}=v_{e i}\left(T_{e}-T_{i}\right)-v_{i n}\left(T_{i}-T_{n}\right)
$$

where

$$
v_{e i}=2.0 \times 10^{-6} \frac{Z_{i} n_{e} \ln \Lambda}{T_{e}^{3 / 2}} \quad\left(\sec ^{-1}\right)
$$

is the Coulomb collision frequency. For $Z=1, n_{e}=10^{10} \mathrm{~cm}^{-3}, \ln \Lambda=10$ and $T_{e}=$ $3 \mathrm{eV}$, we find:

$$
v_{e i}=3.85 \times 10^{4} \quad\left(\sec ^{-1}\right)
$$

Since the neutr $\rightarrow 1$ gas is much colder than the ions, we can set the temperature of neutrals as zero, i.e. $T_{n}=0$. To calculate $v_{i n}$, the collision frequency between the ions and the neutrals, a classical model is used which gives the following relation: 


$$
v_{i n}=n_{0} \sigma_{0} v_{i t h}=n_{0} \sigma_{0}\left(\frac{T_{i}}{m_{i}}\right)^{1 / 2}
$$

where $n_{0}$ is the neutral gas density and $\sigma_{0}$, which is about $5 \times 10^{-15} \mathrm{~cm}^{2}$, is almost independent of the electron temperature.

Considering a "cold" initial condition of the ion temperature (i.e. $T_{i}(t=0)=0$ ), then we can get the ion temperature as a function of time $t$ :

$$
T_{i}(t)=\frac{v_{e i} T_{e}}{v_{e i}+v_{i n}}\left[1-e^{-\left(v_{e i}+v_{i n}\right) t}\right]
$$

The cold ions will be heated up during the characteristic time $\tau_{\text {tran }}$ in which the ions longitudinally transit through the discharge tube, and can be estimated as follows:

$$
\tau_{\text {tran }}=\frac{L}{v_{i t h}}=L\left[\frac{m_{i}}{T_{i}}\right)^{1 / 2}
$$

then we can express the final ion temperature as:

$$
T_{i}\left(t=\tau_{\text {tran }}\right)=\frac{v_{e i} T_{e}}{v_{e i}+v_{i n}}\left\{1-e^{-\left(v_{e i}+v_{i n}\right) L}\left[\frac{m_{i}}{T_{i}\left[\tau_{\text {tran }}\right]}\right]^{h /}\right\}
$$

The numerical solution for this equation gives:

$$
T_{i}\left(t=\tau_{\text {tran }}\right)=0.2 \quad(e V)
$$

Since the longitudinal ion temperature is about $1(\mathrm{keV})$, then we can get both the parallel and the perpendicular (with respect to the magnetic field line) velocities as: 


$$
\begin{cases}v_{i \downarrow}=4.6 \times 10^{5}(\mathrm{~cm} / \mathrm{sec}) & (\text { at } 0.2(\mathrm{eV})) \\ v_{i \|}=3.1 \times 10^{7}(\mathrm{~cm} / \mathrm{sec}) & (\text { at } 1.0(\mathrm{keV}))\end{cases}
$$

Then the emittance of the beam can be calculated as:

$$
\theta_{c}=\operatorname{tg}^{-1}\left[\frac{v_{i \perp}}{v_{i \|}}\right]=\operatorname{tg}^{-1}\left[\frac{4.6 \times 10^{5}}{3.1 \times 10^{7}}\right]=0.85^{\circ},
$$

which is almost equal to the focusing angle $\theta_{f}=0.87^{\circ}$.

The spot size of a beamlet is:

$$
\Delta x=\Delta y=\operatorname{tg}\left(\theta_{c}\right) L_{N B}=2.5(\mathrm{~cm})
$$

where $L_{N B}=164(\mathrm{~cm})$ is the total path length of the beam transport before injected into the HBT plasma. So we loose about $50 \%$ of the NHB current because the size of the side-port is $d=1.5 \mathrm{~cm}$; therefore the vertical injection from the bottom of the vacuum vessel is preferred. We should remember that there is also a factor $\approx 2 \%$ increasing the perpendicular component of the beam velocity $v_{i \perp}$ by the mirroring effect when an ion moves along the entire length of the neutralizer.

\subsubsection{The Vacuum System}

The vacuum system of the NB injector consists of the puff-gas valve, the discharge tube, the neutralizer, the plenum (i.e. the beer barrel vessel), the retractable NB calorimeter, the insulation valve and the feed tube (see Fig.1.6). When hydrogen 
gas is fed into the discharge tube at the pressure about $10 \mathrm{mTorr}$, by the puff-gas valve, it eventually diffuses into the neutralizer (at about 2 mTorr determined by a pressure aperture) at the thermal speed of room temperature $(-0.1 \mathrm{~cm} / \mu \mathrm{sec}$ for hydrogen atom). The pressure difference between the discharge tube and the neutralizer is maintained by the extraction aperture (with total opening area $0.43 \mathrm{~cm}^{2}$ ) which was divided into 219 smaller apertures (i.e. the multi-aperture extraction structure). In order to minimize the reionization losses, we must pump away the cold target hydrogen gas fror 1 the neutralizer and dump the emerging charged fraction of the beam. The reason is that the fast neutrals can be ionized again when they collide with hydrogen molecules or atoms, and as charged particles are deflected to the injector walls by the fringing magnetic field of the HBT plasma. A turbo-molecular pump, which is connected to the plenum, is used to provide a pumping speed about 500 liters per second. Another use of the plenum (i.e. the beer barrel) is to dump the plasma which comes from the opening of the insulation valve to isolate the NHB from the preionization before it injects into the HBT chamber.

The timing sequence of the NHB system is shown in Fig.1.16. Since the pressure in different parts of the system varies on a $50 \mathrm{msec}$ time scale which is much longer than the time scale of guiding magnetic field $(1 \mathrm{msec})$ and the beam pulse duration 
$(20 \mu \mathrm{sec})$, therefore the pressure variation is neglected during a firing of NHB shot.

\subsection{The Test of the NHB Injector System}

The firing circuit of the cold discharge tube is shown in Fig.1.17. The vacuum test result is presented in Fig.1.18 which shows that the pulser can provide up to $-1.8 k V$ voltage for the discharge tube and the net accelerating bias voltage is about $-1.6 k V$ due to some leakage of the circuit. A constant repelling bias voltage $(-1.1 k V)$ is provided by a DC voltage source(battery) to prevent the electrons born in the neutralizer from going back to the source plasma region.

When the system was filled with hydrogen gas through the puff gas valve, both current and voltage signals are measured for each electrode as shown in Fig.1.19. The three pictures were taken from three different shots under the the same operating condition since we can measure only one pair of current and voltage signal in each shot. In each picture, the top one is the discharge current in the discharge tube, and the bottom one is the voltage applied on each electric grid, respectively.

The discharge process includes threz different periods: the no-discharge period, the preion period and the discharge period. The duration of the three processes is different from shot to shot and is mainly determined by and very sensitive to the fill pres- 
sure in the discharge tube.

First in the no-discharge period, the voltage across the two electrodes in the discharge tube is $-1.8 \mathrm{kV}$ and maintained constantly. Now the working gas is neutral and only few residual are accelerated.

Secondly in the preion period, the accelerated electrons formed in previous period collide with neutrals resulting in many more electrons and ions. Now the working gas is partially ionized and somewhat conductive. Therefore the discharge voltage decreases to $-1.2 k V$ depending on the ionization degree.

Finally in the discharge period, the working gas is almost fully ionized in the central region and the discharge voltage decreases to $-50 \mathrm{~V}$. The discharge current keeps almost the same waveform as that of the vacuum discharge voltage and eventually drops as the voltage drops to zero. The transition from one period to next happens in a time scale shorter than a microsecond since the ionization process resulted from electron impact collision happens in $10^{-8}$ second time scale. Fig.1.19(a) shows that a constant $13.6 \mathrm{~A}$ discharge current can be maintained as long as $60 \mu \mathrm{sec}$ and then starts decreasing.

The discharge current and the bias voltage on the accelerating screen(with $80 \%$ transparency) are shown in Fig.1.19(b). In the no-discharge period, the bias voltage is 
$-1.6 k V$ and is the same as that in vacuum test case. However, in the preion period, the voltage drops to $-1.58 \mathrm{kV}$ and to $-1.55 \mathrm{kV}$ in the discharge period because the screen picks up some current. From the voltage drop and the screen transparency, the total current extracted from the discharge tube is $I_{\text {acel }}^{+}=1.6 \mathrm{~A}$ in which $0.32 \mathrm{~A}$ is picked up by the screen and $1.28 \mathrm{~A}$ passes through the screen. Since the decelerating screen has also $80 \%$ transparency, the total current transferred into the neutralizer is only $1.024 \mathrm{~A}$. As the pressure in the neutralizer is not low enough here to reduce the intense scattering of the fast ion beam by the background gas, the beam intensity after passing the neutralizer is below a measurable value. Therefore an improvement of exactly controlling the neutralizer pressure is needed.

In Fig.1.19(c), the decelerating voltage is kept constantly at $-1.0 \mathrm{kV}$ because of the usage of a $50 \mu f$ capacitor.

\subsection{The Measurement in the NB experiment}

\subsubsection{The Confinement Time of Fast Ions in the HBT}

Using a fast particle detector (e.g. secondary electron detector, channeltron electron multiplier, silicon surface-barrier detector, etc.), we can measure the fast neutrals escaping from the HBT plasma. 
If we denote $n_{t}(t)$ as the trapped NB particle density in $\mathrm{cm}^{-3}, V$ the total volume of the vacuum vessel in $\mathrm{cm}^{3}$ (note that for HBT chamber $V=45.2$ liters), $\eta$ the trapping efficiency, $\Gamma$ th: incident NB flux in $\mathrm{cm}^{-2} \mathrm{sec}^{-1}$ and $S$ the area of the side port, then the total number of trapped NB particles $n_{t}(t) V$ obeys the following continuity equation:

$$
\frac{d}{d t}\left[n_{t}(t) V\right]=(S \Gamma \eta)-\frac{n_{t}(t) V}{\tau_{l}}-\frac{n_{t}(t) V}{\tau_{t h}},
$$

where $\tau_{l}$ is the loss time of NB particles, and $\tau_{t h}$ is the thermalization time of fast ions.

The loss time $\tau_{l}$ depends on the orbit detrapping, the ripple level of the toroidal magnetic field, the charge exchange of fast neutrals with the relative slow plasma ions, the non-resonant neutralization with plasma electrons, the plasma MHD instabilities, etc.

During the injection period of NB (i.e. $\Gamma \neq 0$ ), the solution of equation (1.24) is:

$$
n_{t}(t) V=\frac{S \Gamma \eta}{\left[\frac{1}{\tau_{l}}+\frac{1}{\tau_{t h}}\right]}\left[1-e^{-t\left(\frac{1}{\tau_{l}}+\frac{1}{\tau_{u h}}\right)}\right]\left(0 \leq t \leq \tau_{p}\right)
$$

where $\tau_{p}$ is the pulse duration time of the NHB which can be adjusted from a few to several hundreds of micro-second. When $t \geq \tau_{p}$, i.e. after the injection of the NHB, since now $\Gamma=0$, equation (1.24) has the following solution:

$$
n_{t}(t) V=\frac{S \Gamma \eta}{\left[\frac{1}{\tau_{l}}+\frac{1}{\tau_{t h}}\right]}\left[e^{\tau_{p}\left(\frac{1}{\tau_{l}}+\frac{1}{\tau_{t h}}\right)} \ldots 1\right] e^{-t\left(\frac{1}{\tau_{l}}+\frac{1}{\tau_{t h}}\right)} \quad\left(\tau_{p} \leq t \leq \infty\right)(1.26)
$$


Fig.1.20 gives the explanation and relations of these quantities.

WE can make an estimate of the signal strength. Suppose $\tau_{l} \approx 1 \sim 2 \mu \mathrm{sec}, \tau_{t h}=$ $95 \mu \mathrm{sec},\left[\frac{\tau_{l} \tau_{t h}}{\tau_{l}+\tau_{t h}}\right] \approx \tau_{l}, \eta=80 \%$ and $I_{i}=0.5 \mathrm{~A}$, then the number density of the trapped fast ions is approximately expressed as:

$$
n_{t}(t)=\frac{I_{i} \tau_{l} \eta}{e V}=4.3 \times 10^{8} \quad\left(\mathrm{~cm}^{-3}\right)
$$

In order to estimate the signal strength that finally we can get from the detector, for example, the secondary electron detector, we should first calculate the total number of the trapped fast ions resulting from the NHB injection per second:

$$
\frac{d}{d t}\left[n_{i}(t) V\right]=S \Gamma \eta=\frac{S J}{e} \eta=\frac{I \eta}{e}=2.5 \times 10^{8} \quad(\text { particles } / \text { second })
$$

For each incident neutral hydrogen atom striking the collector surface, $\gamma$ secondary electrons will be produced where $\gamma$ is the secondary electron emission coefficient of the detector surface and depends on the energy of the incident particle, the surface material and the surface condition. For aluminum surface at $1 \mathrm{keV}$ incident energy (which is corresponding to the velocity of $v_{b}=4.4 \times 10^{7} \mathrm{~cm} / \mathrm{sec}$ ), $\gamma=0.5$. Then the sigral current can be estimated as:

$$
\begin{aligned}
& \text { Signal current } \approx\left[\frac{d}{d t}\left[n_{t}(t) V\right]\right]\left[\frac{S}{S_{V}}\right] \gamma \\
& =2.175 \times 10^{14}(\text { electrons/second })
\end{aligned}
$$




$$
\begin{aligned}
& =3.48 \times 10^{-5}(\text { Coulomb/second }) \\
& =34.8(\mu A),
\end{aligned}
$$

where $S_{V}=10193.8 \mathrm{~cm}^{2}$ is the total surface area of the vacuum vessel, and $S=$ $1.767 \mathrm{~cm}^{2}$ is the target area of the detector which has an aluminum collector. So we can still pick up a several tens $m V$ signal when the detector current is passed through a $1 k \Omega$ termination resistor.

\subsubsection{The Detection of Instabilities}

The NHB probe can also be used to examine some macroscopic MHD instabilities, or the failure of magnetic symmetry, etc. When there is no instability, the signal from the current monitor located on the inside wall of the torus should be relatively smooth and we can measure the effective loss time of fast ions from the shape of the curve, as shown in Fig.1.21 (a). But when an instability happened, the signal curve will be suddenly dumped and will not be smooth, as shown in Fig.1.21(b). From this we can get the time at which the instability happened.

\section{Acknowledgements:}

We sincerely thank Professor G. A. Navratil and Professor M. E. Mauel for their 
valuable discussions.

This work was supported by U.S. Department of Energy under contract No. DEFG02-86ER53222.

\section{References:}

[1] Menon, M. M., "Neutral Beam Heating Applications and Development", Proceedings of IEEE, Vol.69, No.8, August 1981, p.1012-1029.

[2] Kunkel, W. B., "Neutral-Beam Injection", p.104-150.

[3] Hemsworth, R. S., "Neutral Injection Plasma Heating", Plasma Physics and Nuclear Fusion Research, U.K.A.E.A. Research Group, Culham Laboratory, Academic Press, London, 1981, p.455-476.

[4] Tsai, C. C., et al., "Determination of Species Yield of Ion Sources Used for Intense Neutral Beam Injector", Journal of Nuclear Materials 111 and 112, North-Holland Publishing Company, 1982, p.153-158.

[5] Fleischmann, H. H., Barnett, C. F. and Ray, J. A., "Small-Angle Scattering in Stripping Collisions of Hydrogen Atoms Having Energies of $1-10(\mathrm{keV})$ in Various gases", Physical Review A, Volume 10, Number 2, August 1974, p.569-583. 
[6] Navratil, G. A., et al., "Experimental and Theoretical Studies of Circular Cross Section High-Beta Tokamak", The Eleventh International Conference on Plasma Physics and Controlled Nuclear Fusion Research 1986, IAEA-CN-47/A-V-4, Kyoto, Japan, 1986.

[7] Nicholson, D. R., Introduction to Plasma Theory, John Wiley and Sons, New York, 1983.

[8] Krall, N. A. and Trivelpiece, A. W., Principle of Plasma Physics, San Francisco Press, Inc., San Francisco, 1986.

[9] Chen, F. F., Introduction to Plasma Physics and Controlled Fusion, Volume 1: Plasma Physics, Second Edition, Plenum Press, New York, 1984.

[10] Lawson, J. D., The Physics of Charged Particle Beams, Clarendon Press, Oxford, 1977.

[11] Miller, R. B., An Introduction to the Physics of Intense Charged Particle Beam, Plenum Press, New York, 1982.

[12] Riviere, A. C., "Penetration of Fast Hydrogen Atoms into a Fusion Reactor Plasma", Nuclear Fusion 11, 1971, p.363-369.

[13] Green, T. S., "Beam Formation and Space Charge Neutralisation", IEEE Tran- 
sactions on Nuclear Science, Vol. NS-23, No.2, April 1976, p.918-928.

[14] Tsai, C. C., Stirling, W. L. and Ryan, P. M., "Plasma Studies on a duoPIGatron Ion Source", Rev. Sci. Instrum., Vol.48, No.6, June 1977, p.651-655.

[15] Grisham, L. R., Tsai, C. C., Whealton, J. H. and Stirling, W. L., "Effect of Emission Aperture Shape upon Ion Optics", Rev. Sci. Instrum., Vol.48, No.8, August 1977, p.1037-1041.

[16] Meixner, C. N., Menon, M. M., Tsai, C. C. and Whealton, J. H., "Geometrical Effects on the Beamlet Optics of a Two-Stage Ion Accelerator", J. Appl. Phys. 52(3), March 1981, p.1167-1174.

[17] Whealton, J. H., Grisham, L. R., Tsai, C. C. and Stirling, W. L., "Effect of Preacceleration Voltage upon Ion-Beam Divergence", J. Appl. Phys. 49(6), June 1978, p.3091-3101.

[18] Guthrie, A. and Wakerling, R. K., Vacuum Equipment and Technique, McGrawHill Book Company, Inc., New York, 1949.

[19] Moriette, P. R., A Study of Strong Transverse Ionizing Shorck Waves, PhD Thesis, Report No.56, Columbia University, 1971. 


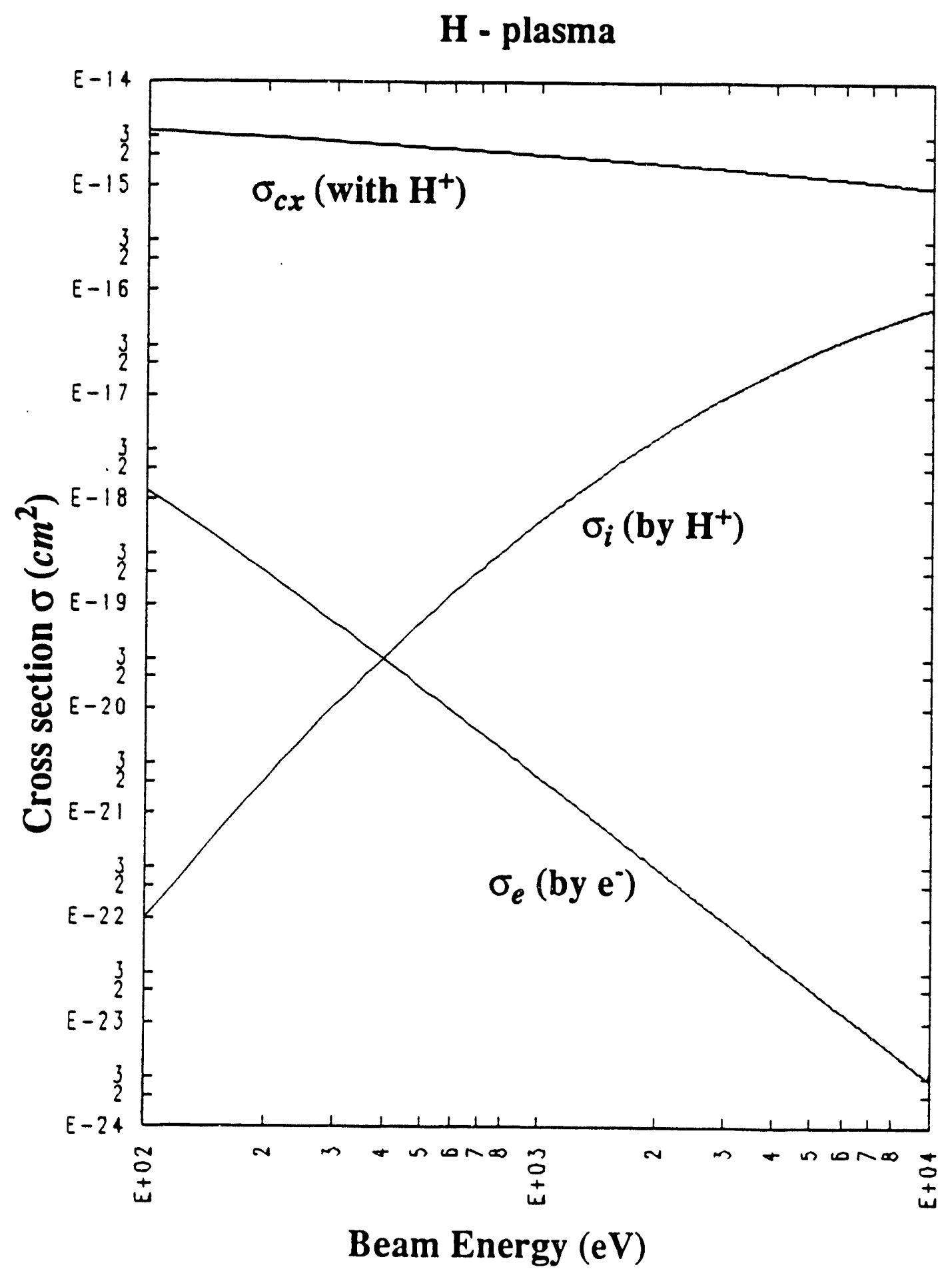

Figure 1.1 The effective collision cross section of neutral hydrogen beam in a hydrogen plasma by different atomic processes. 


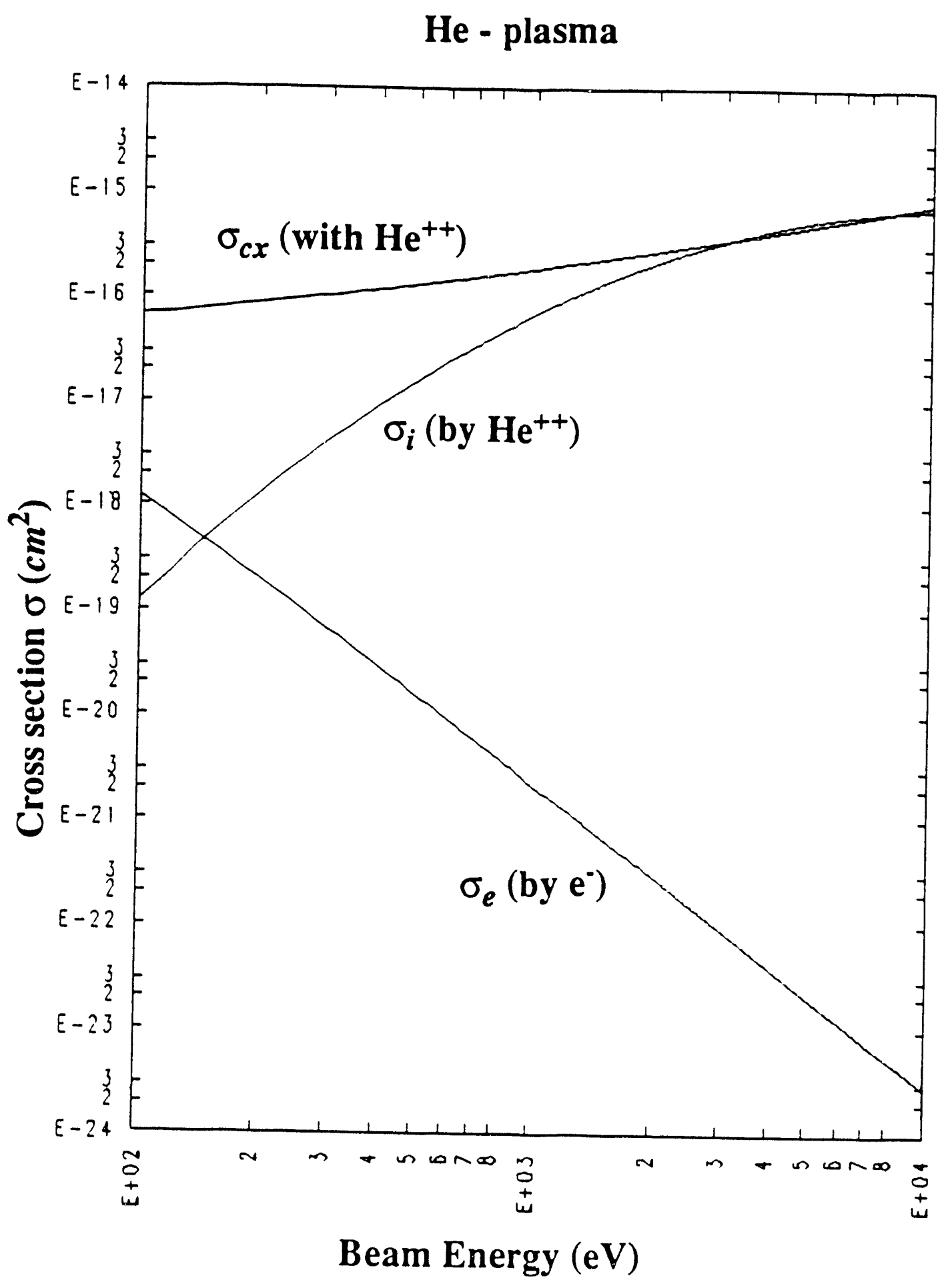

Figure 1.2 The effective collision cross section of neutral hydrogen beam in a helium plasma by different atomic processes. 

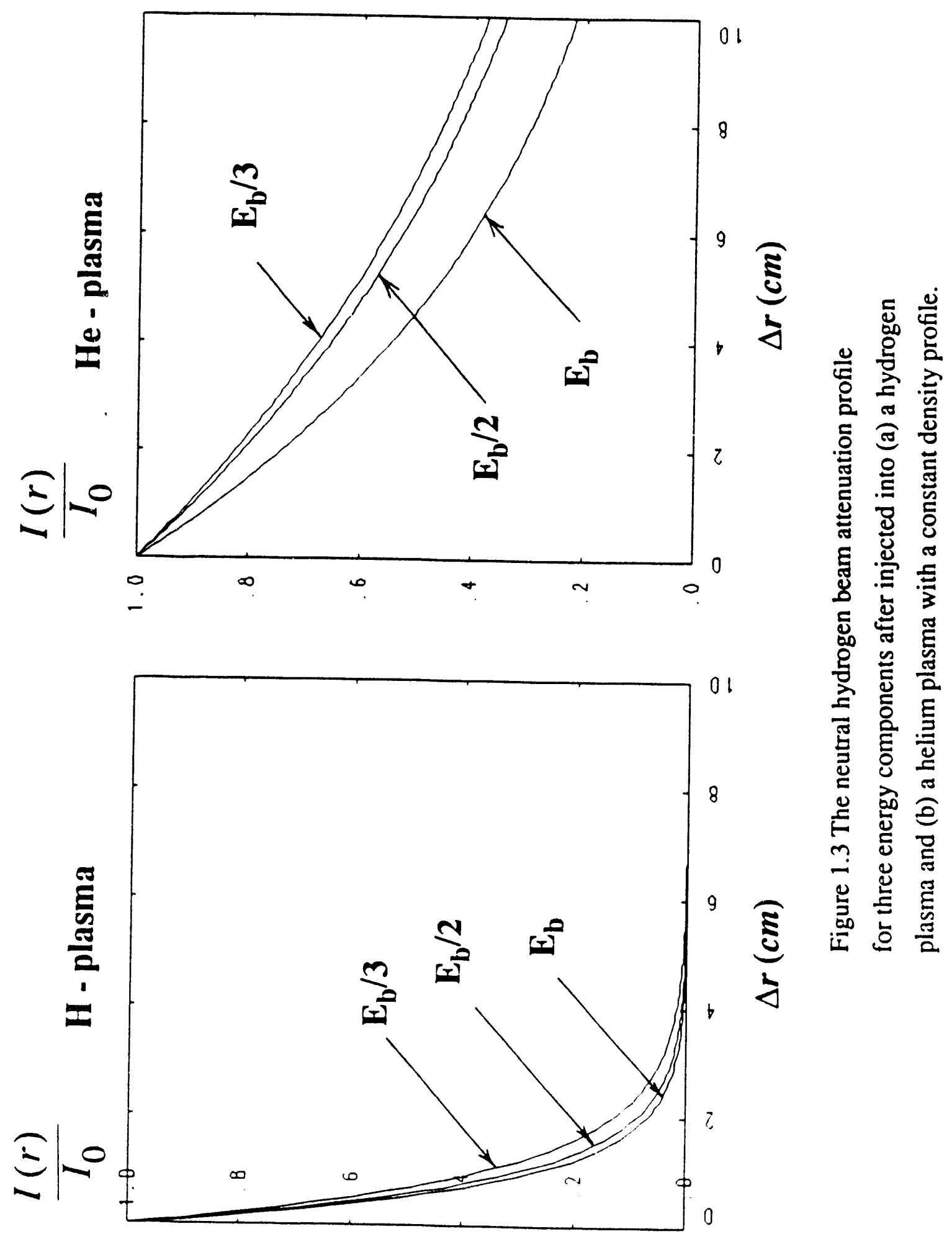

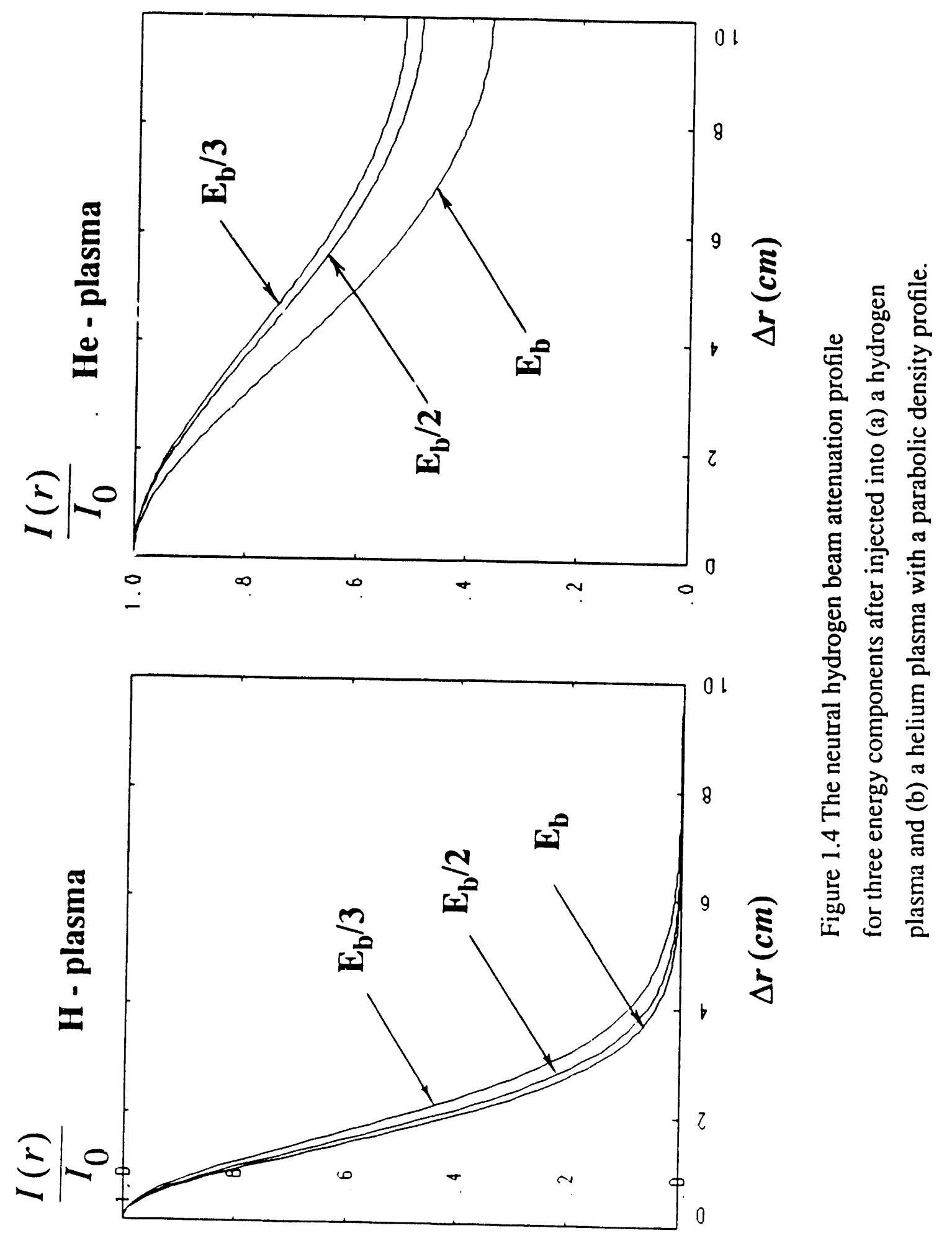


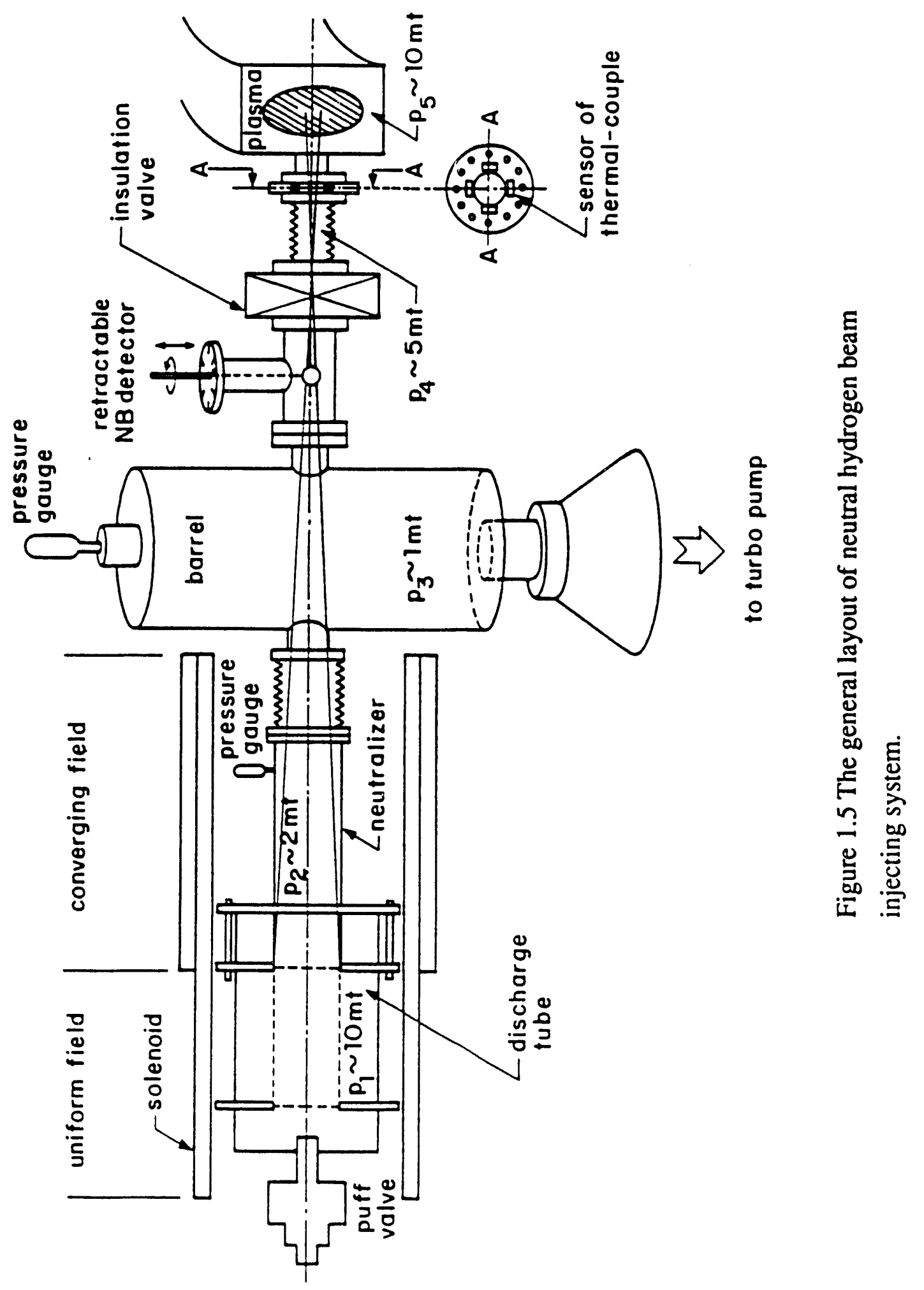




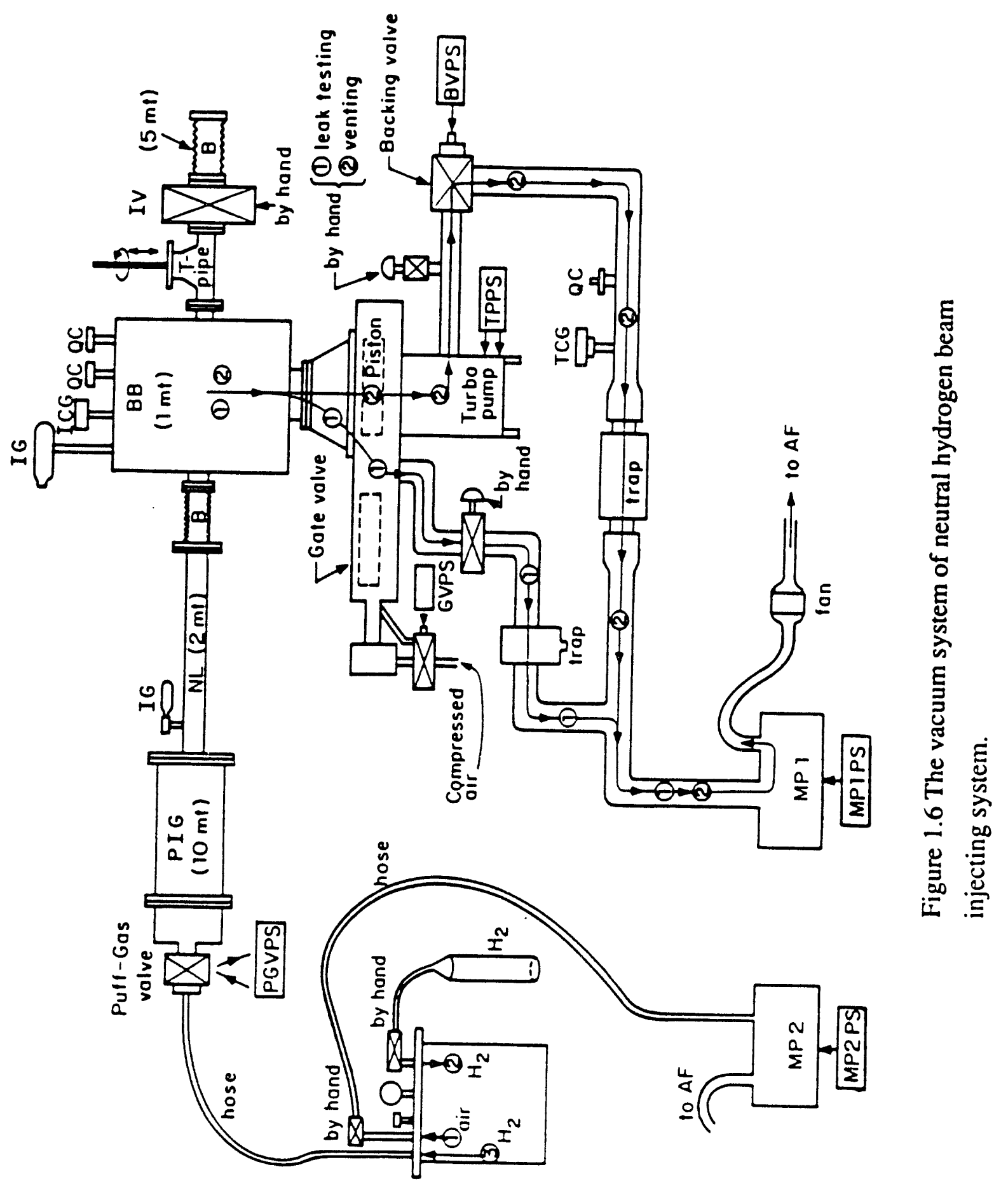




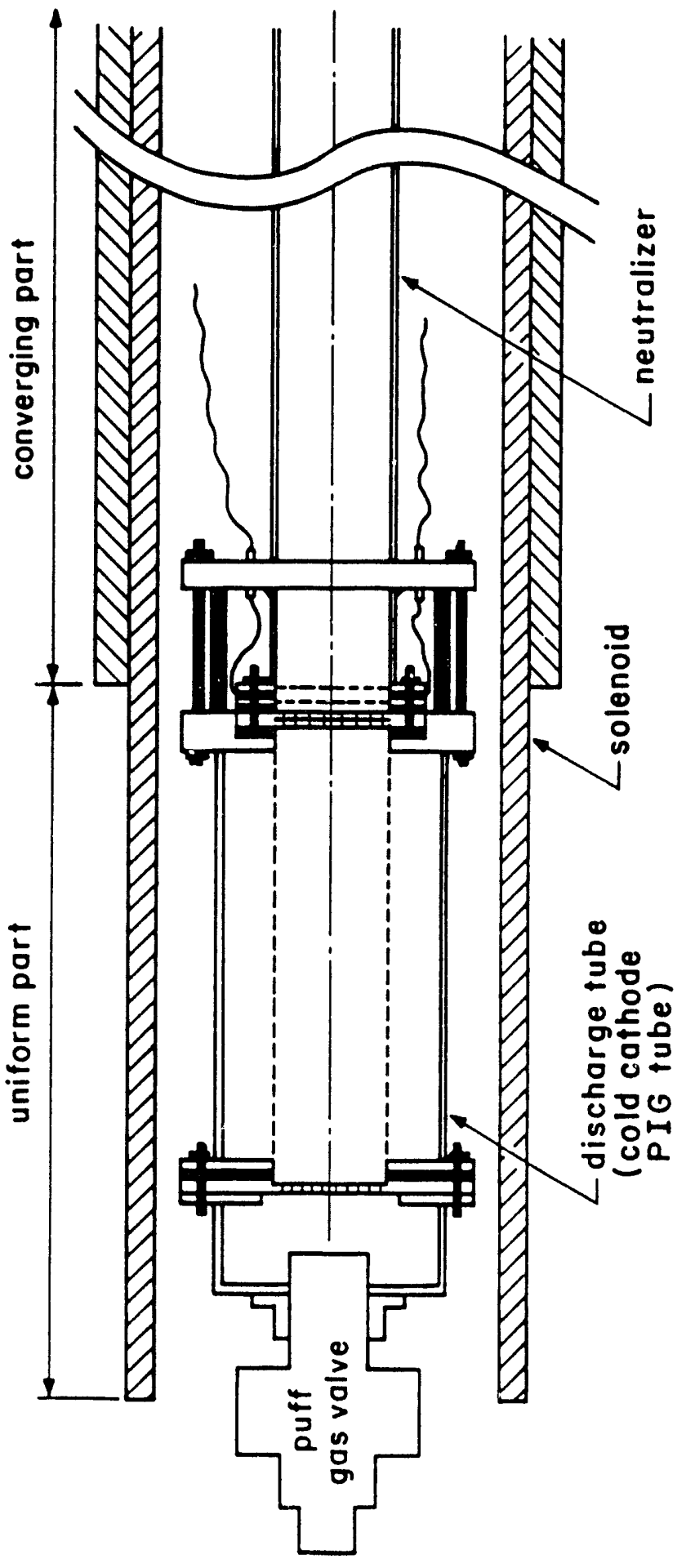

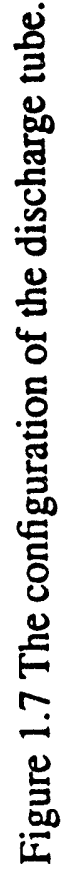






Figure 1.8 The configuration of the puff gas valve. 

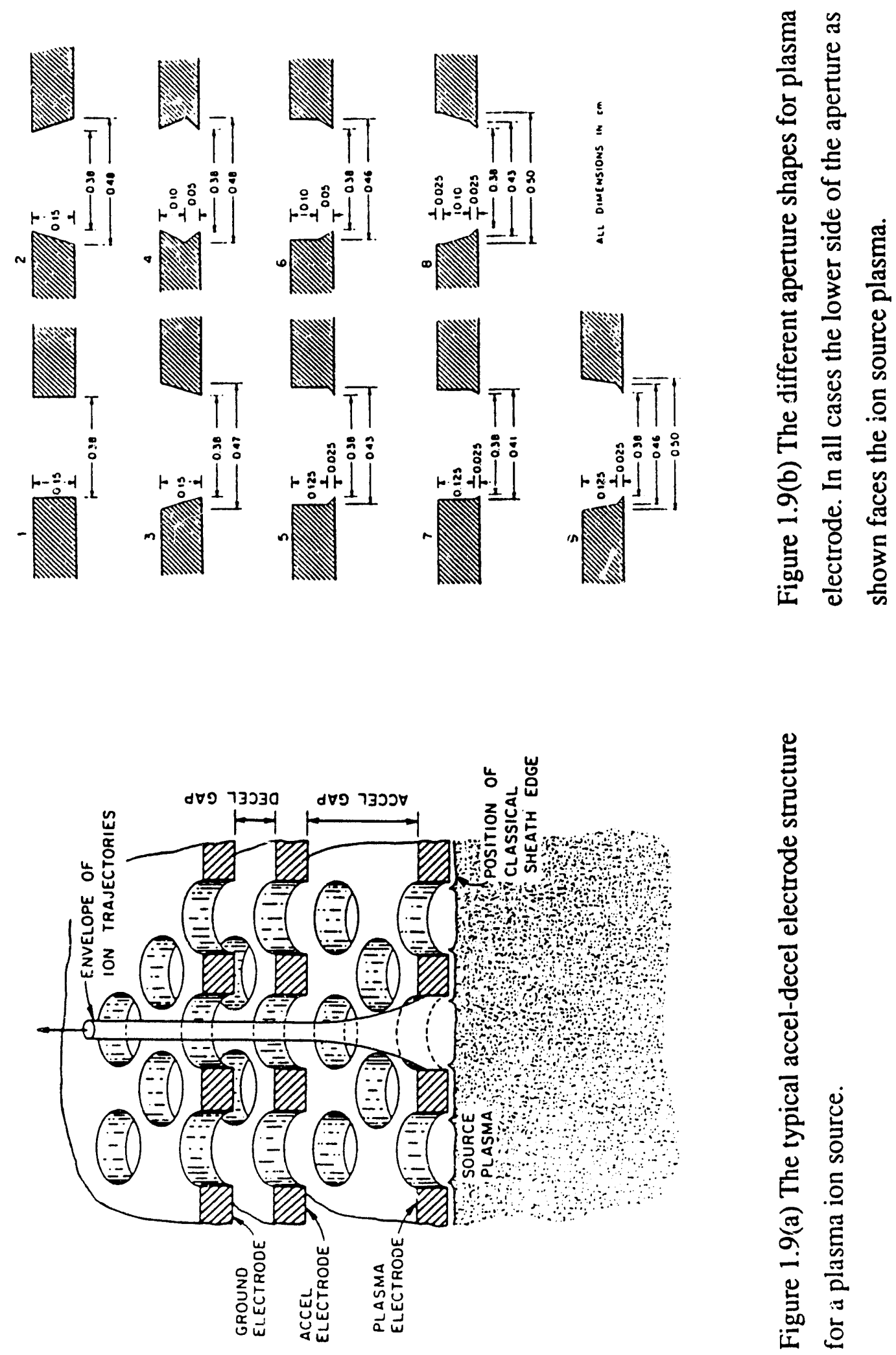


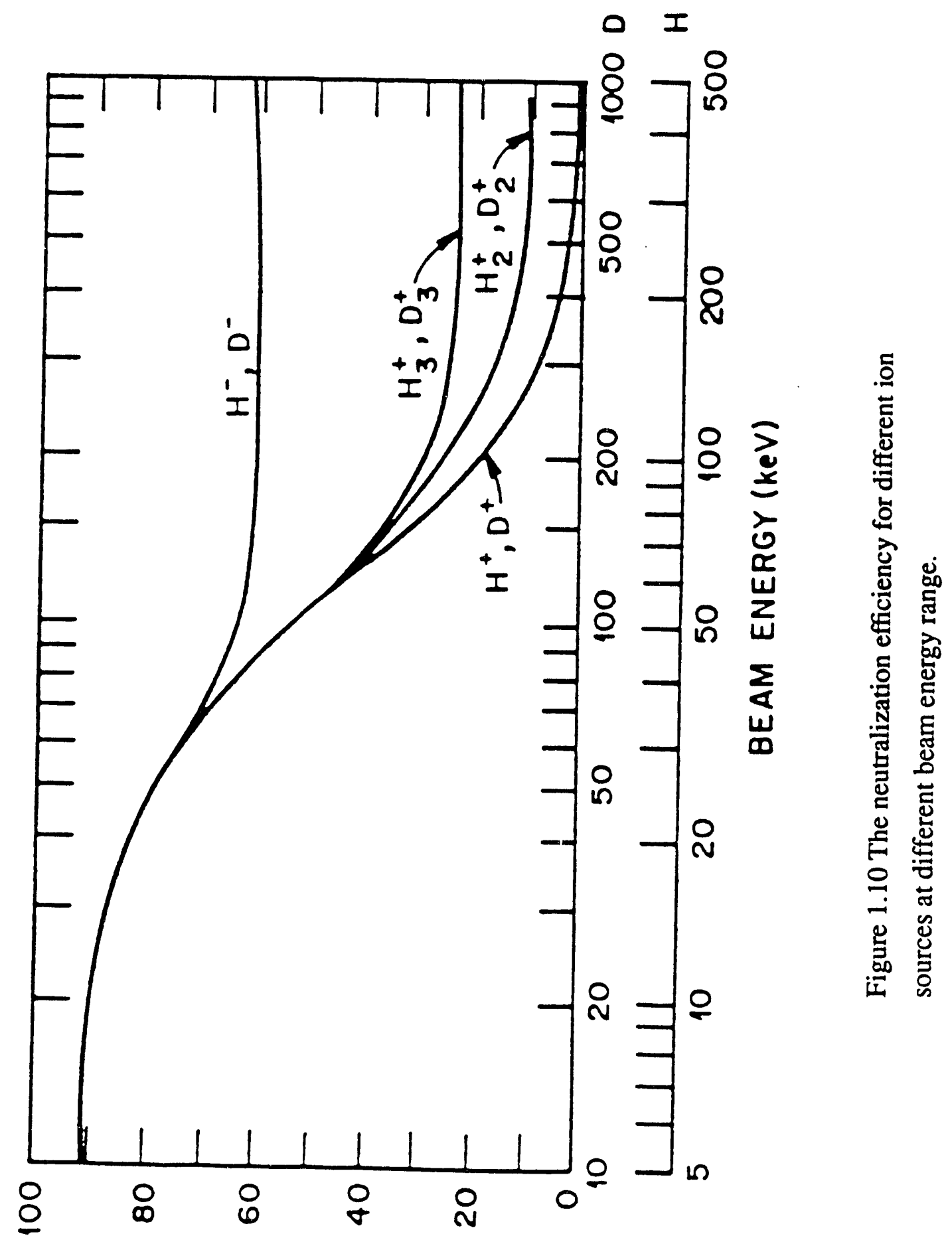

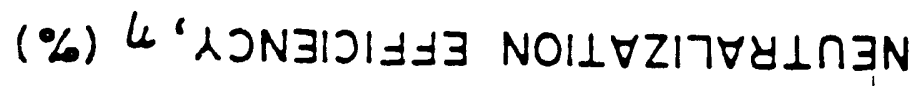




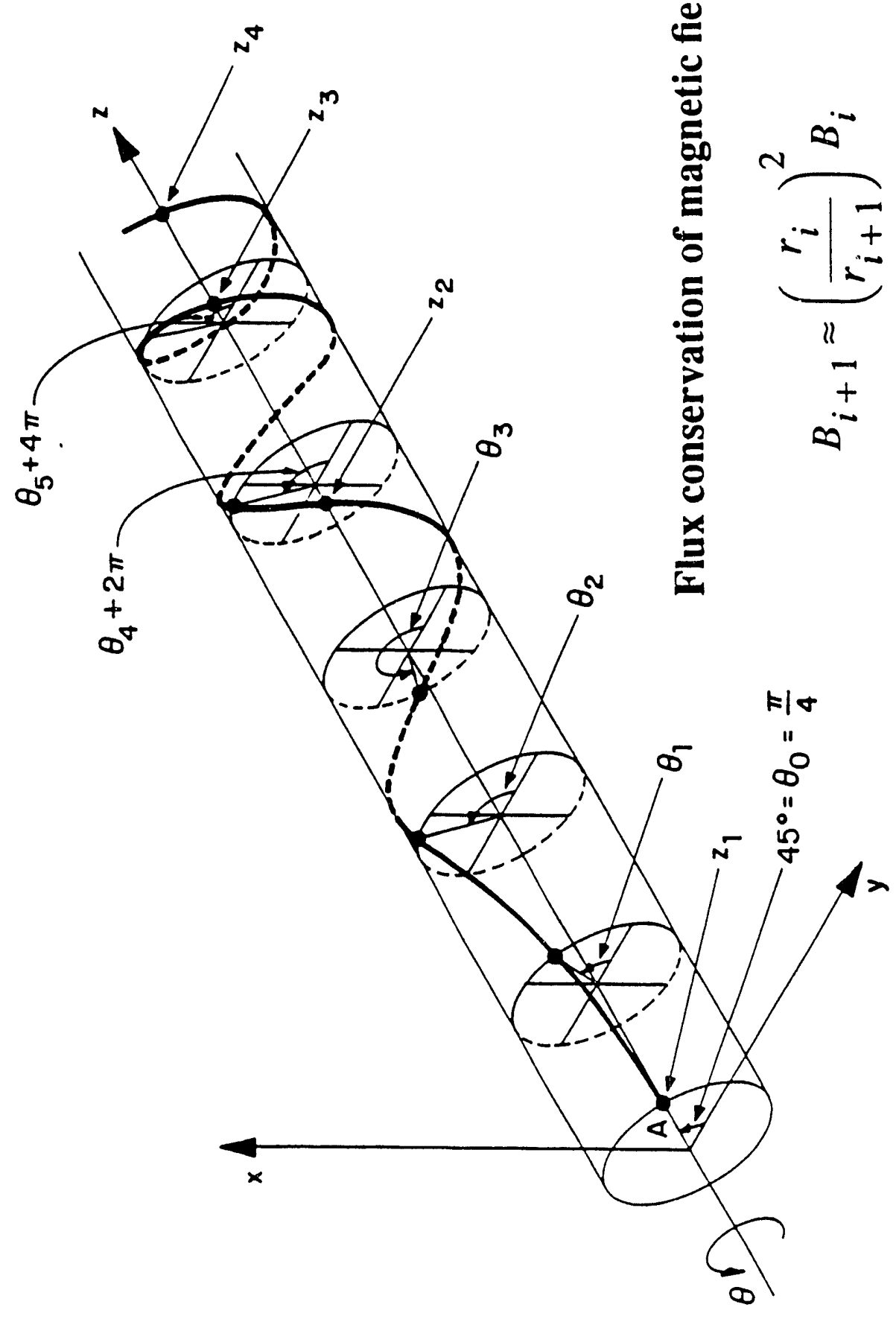

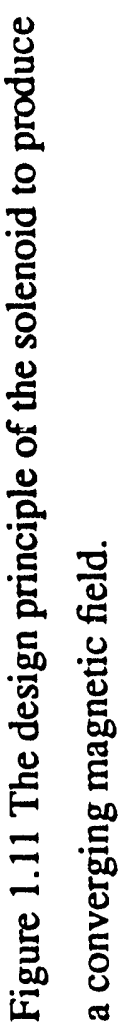




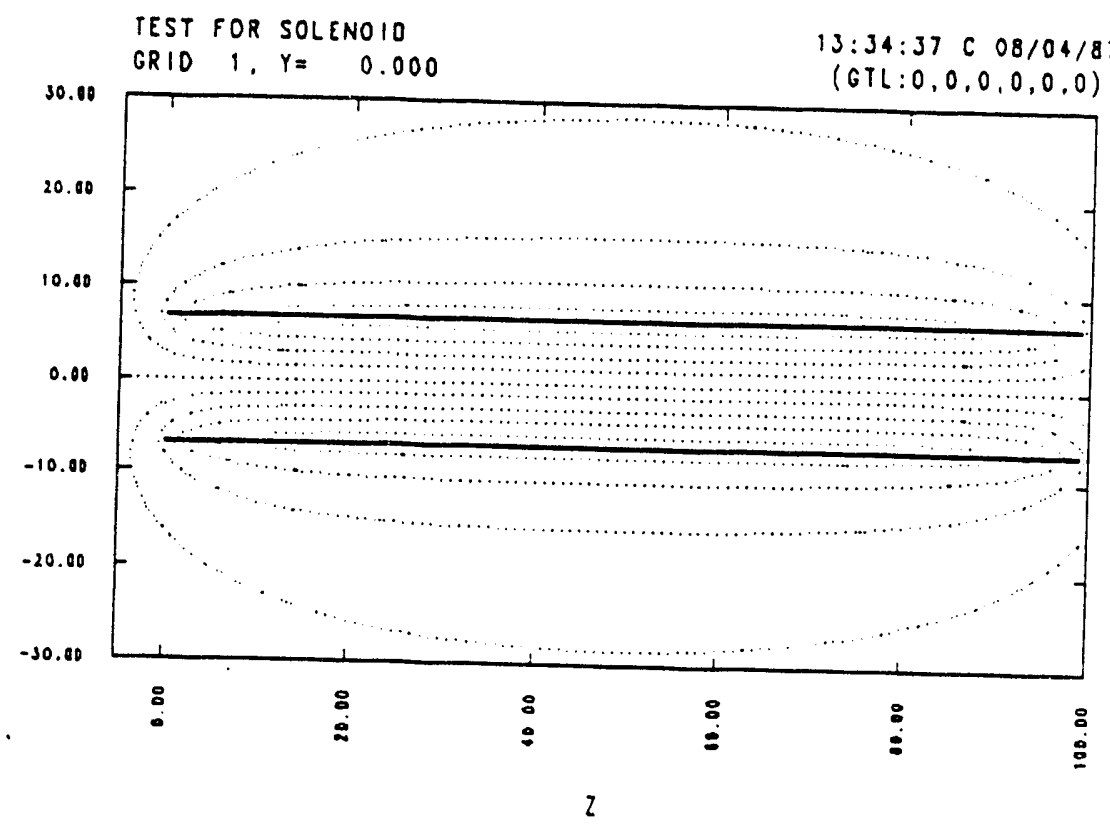

A-CONTOURS

10.

$22.500 E+02$

$35.000 E+02$

$47.500 E+02$

$51.000 E+03$

TEST FOR SOLENOIO

FIELD MAGNITUDE ALONG FLUX LINES, IHROUGH $14: 37$ C 08/04/87

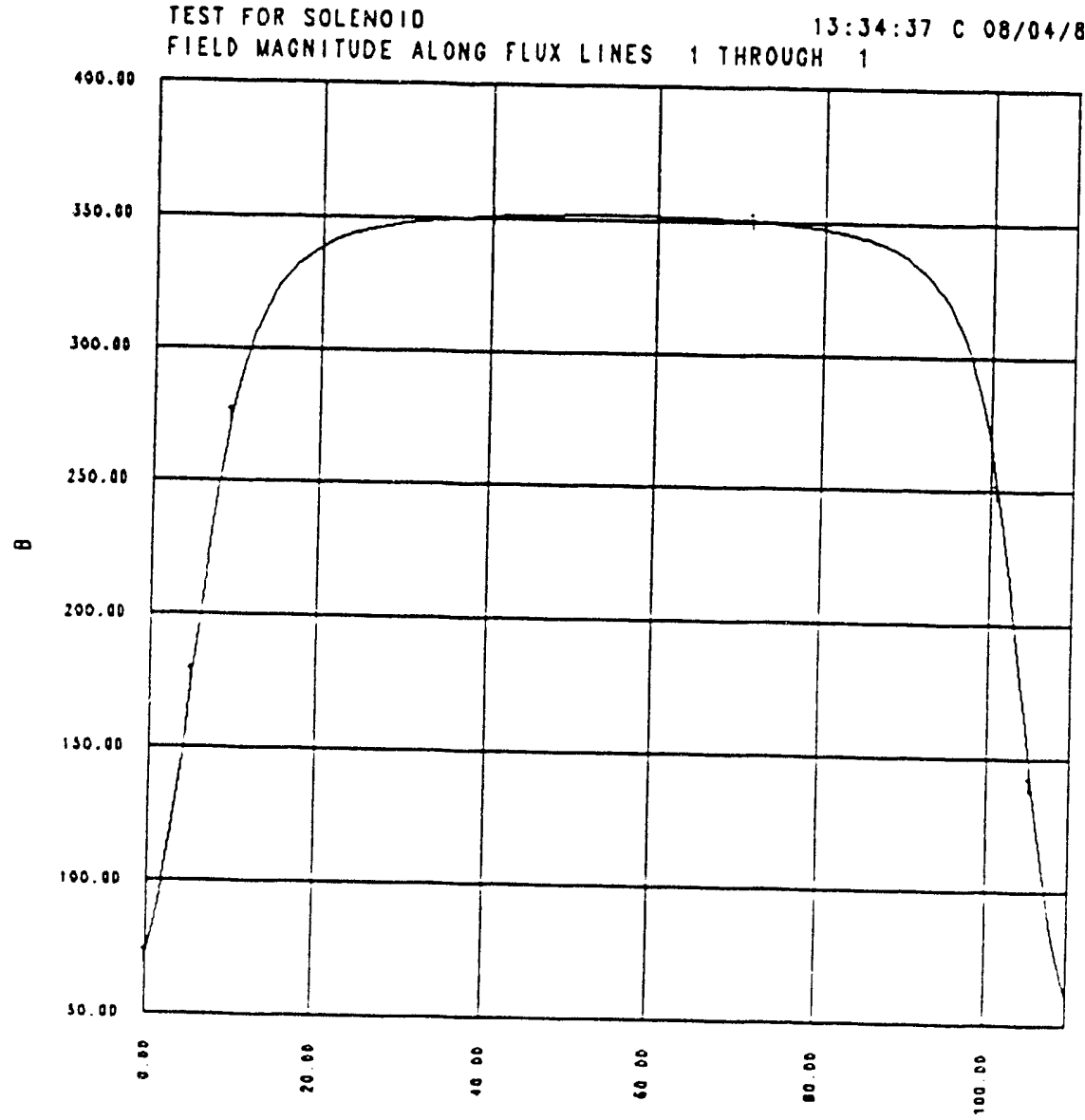

Figure 1.12 The flux contour and field strength produced by an uniformly winded solenoid. 

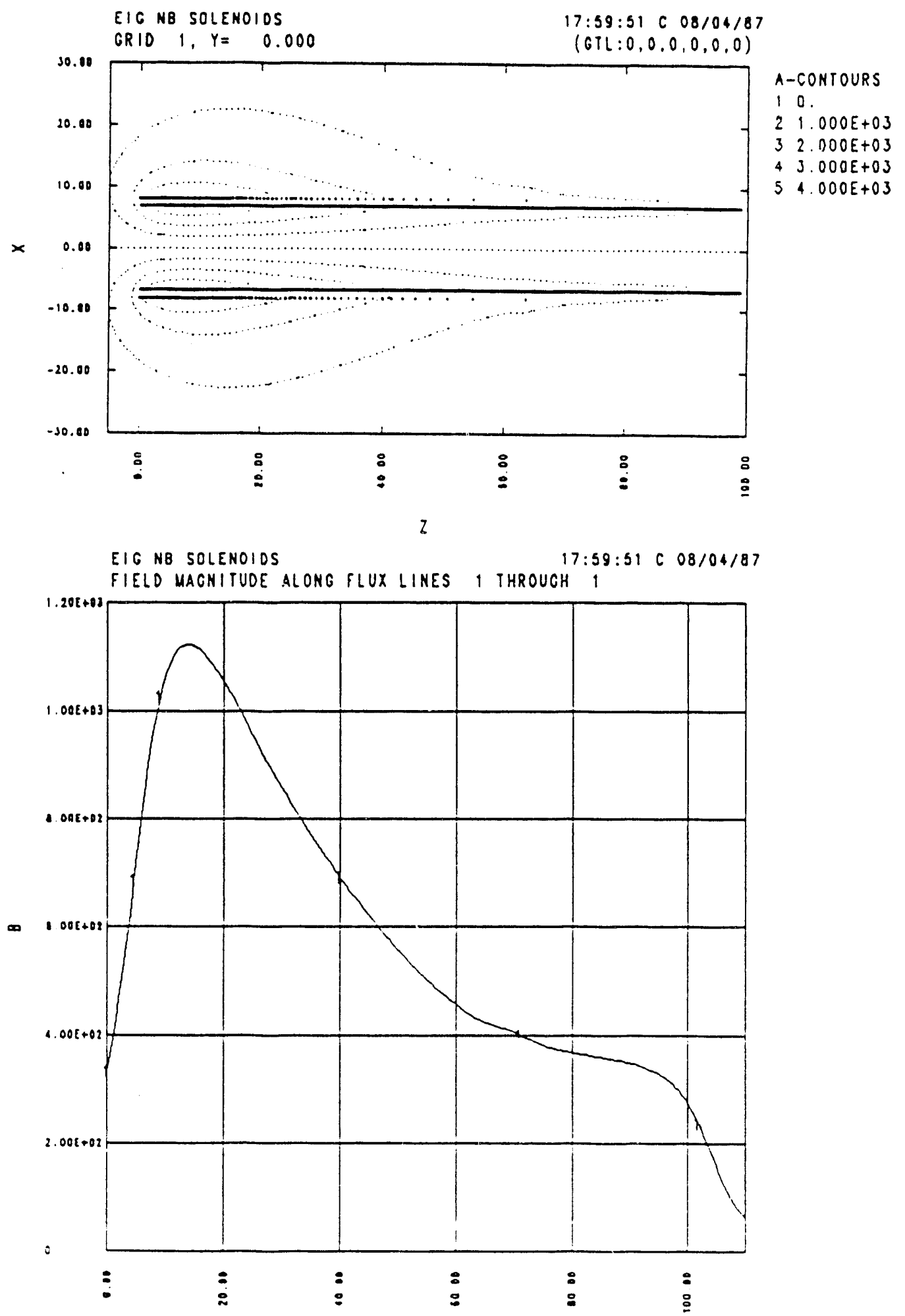

Figure 1.13 The flux contour and field strength produced by an uniformly and a non-uniformly winded solenoids. 

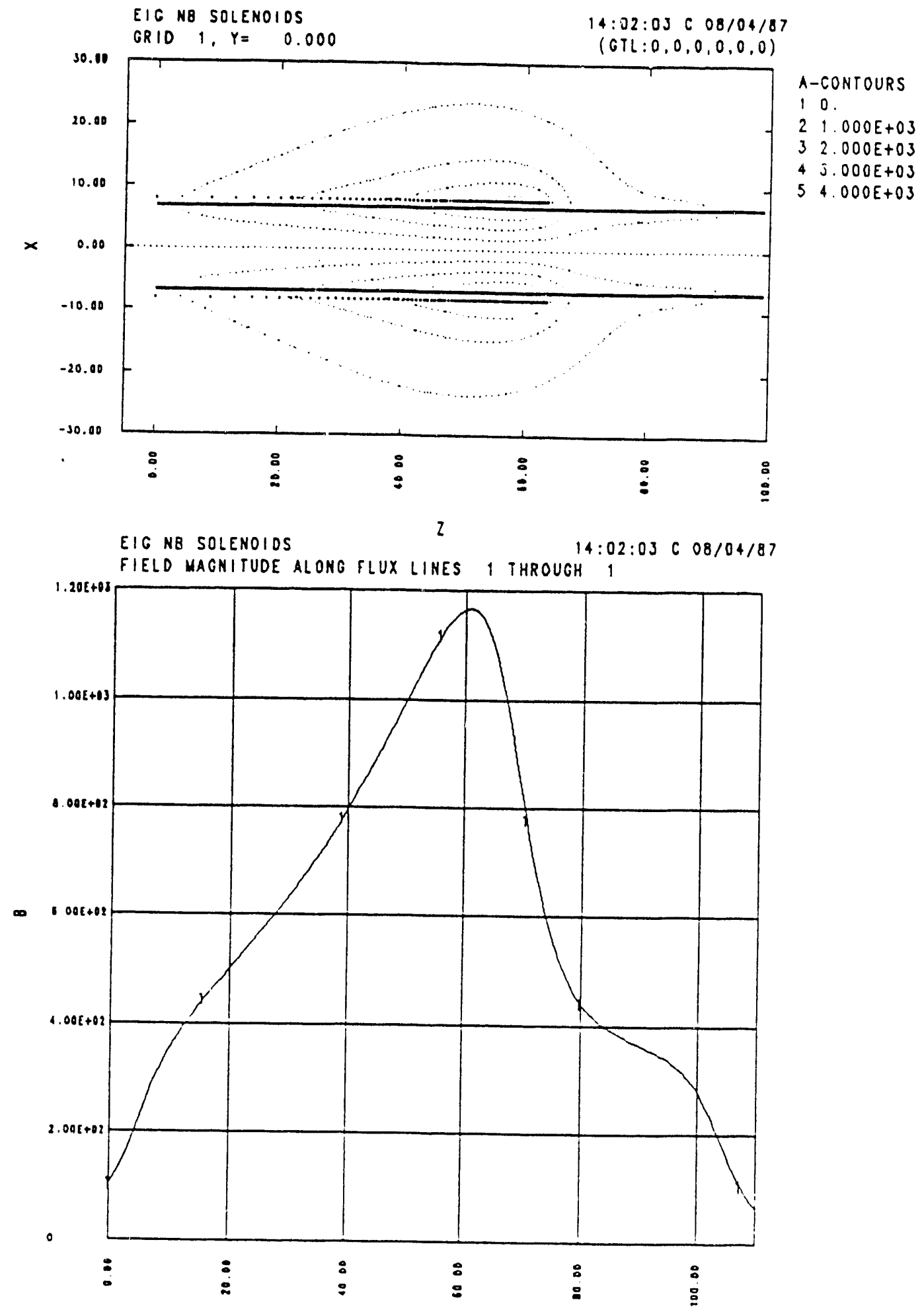

Figure 1.14 Similar to that shown in Fig. 1.13 except that now the non-uniformly winded solenoid is put in the opposite direction. 


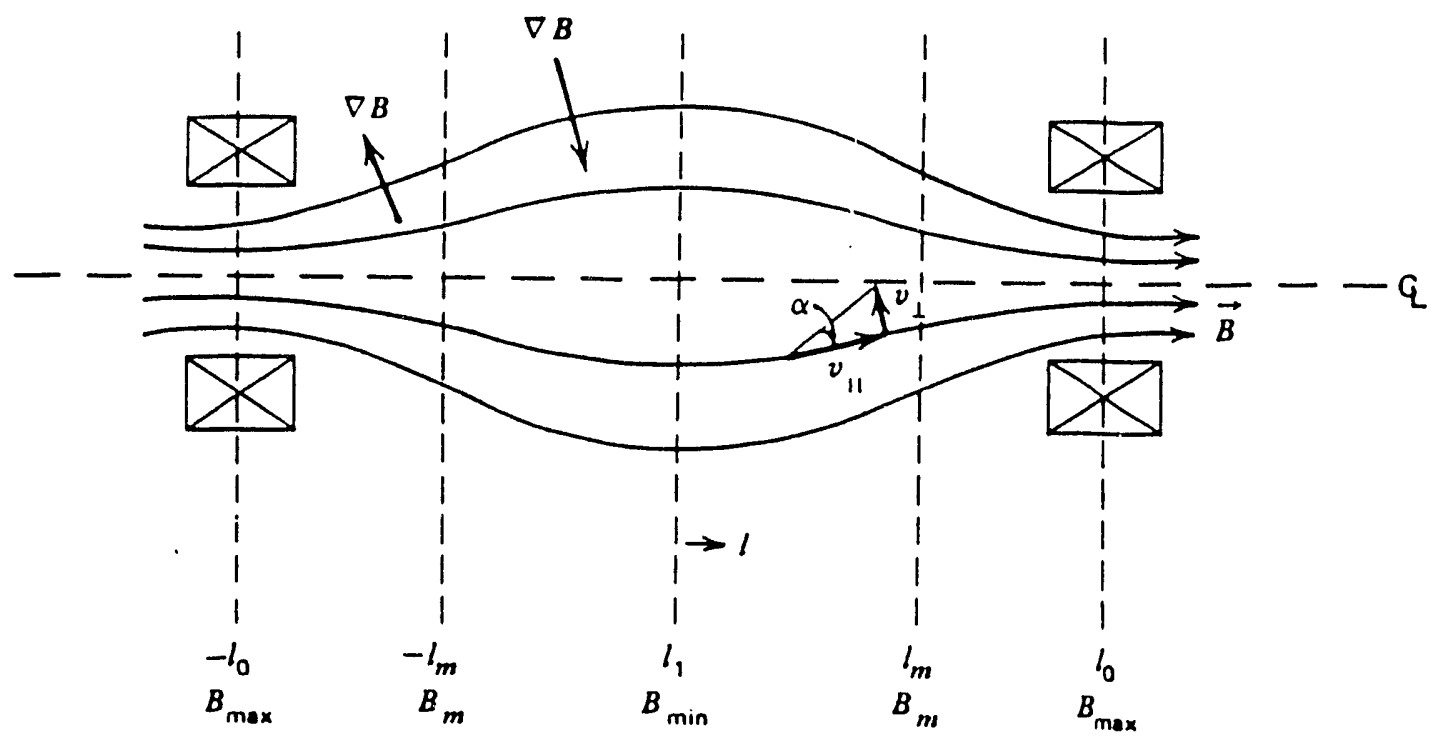

The "pitch" angle

$$
\tan \alpha(l) \equiv \frac{v_{\perp}(l)}{v_{\|}(l)}=\frac{\sqrt{W_{\perp}(l)}}{\sqrt{W_{\|}(l)}}
$$

The magnetic moment

$$
\bar{\mu} \equiv \frac{W_{\perp}(l)}{B(l)}=\frac{W_{0} \sin ^{2} \alpha(l)}{B(l)}=\text { const } .
$$

The trapping condition

$$
\left(\frac{B(l)}{B_{\max }}\right)^{1 / 2} \leqslant \sin \alpha(l) \leqslant\left(\frac{B(l)}{B_{\min }}\right)^{1 / 2}
$$

Figure 1.15(a) The basic principle of a magnetic mirror. 
Loss cone in velocity space.

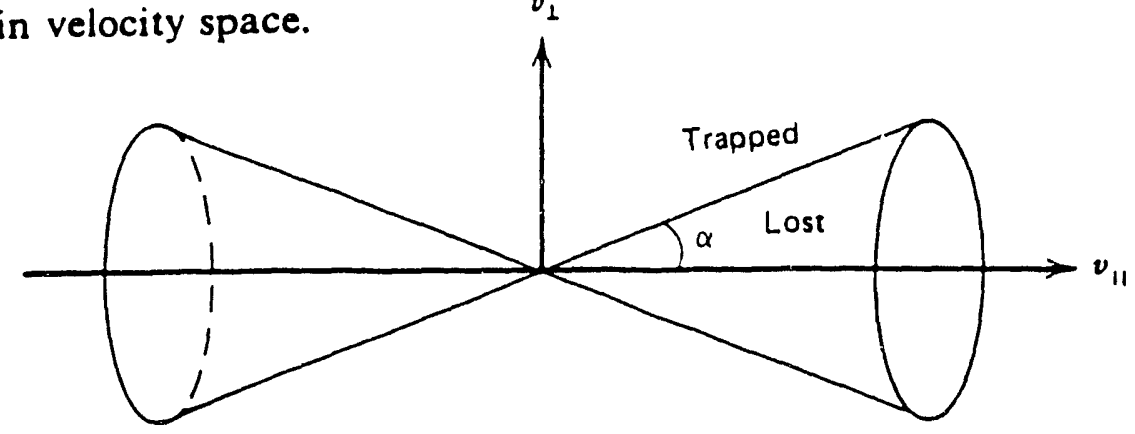

$$
\alpha_{L C}\left(l_{1}\right)=\sin ^{-1}\left(\sqrt{\frac{B_{\min }}{B_{\max }}}\right) \equiv \sin ^{-1}\left(\sqrt{\frac{1}{R_{M}}}\right)
$$

For a collection of particles with a uniform velocity distribution, the escape fraction is

$$
f_{\text {loss }}(l)=\frac{\int_{0}^{\alpha_{L C}} \sin \alpha d \alpha}{\int_{0}^{\pi / 2} \sin \alpha d \alpha}=1-\cos \left(\sin ^{-1} \alpha_{L C}(l)\right)
$$

$$
\text { For } R_{M} \gg 1 \quad f_{\text {loss }}\left(l_{1}\right) \approx \frac{1}{2 R_{M}}
$$

Figure 1.15(b) The definition of loss cone and escape fraction. 

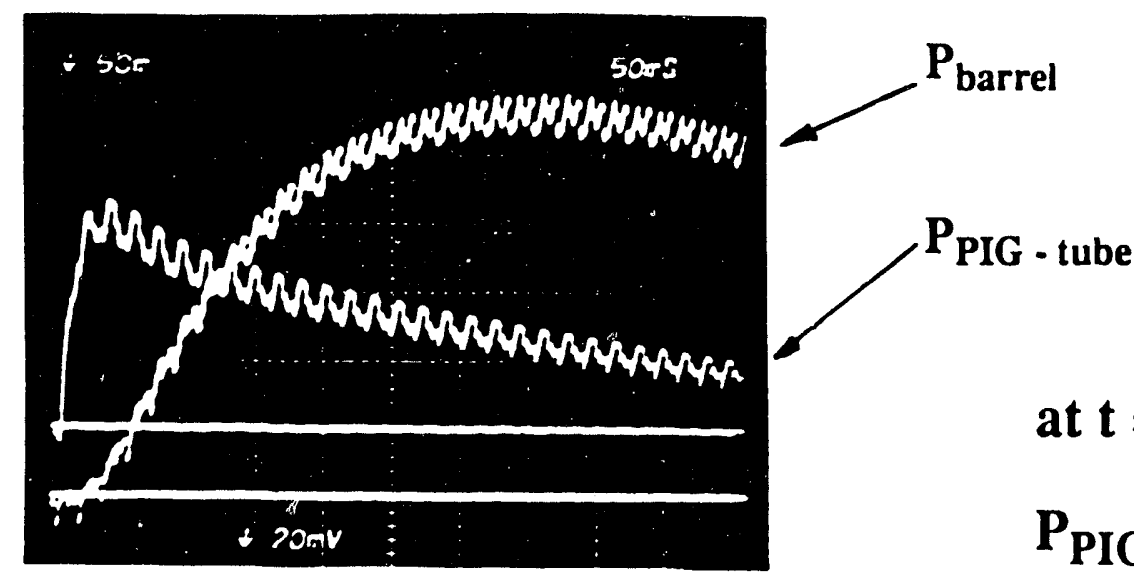

$$
\text { at } \mathrm{t}=\mathbf{1 0 0 . 0}(\mathrm{msec})
$$

PIG - tube $_{\text {PI }} \approx 2.0(\mu)$

$P_{\text {neutralizer }} \approx 1.7(\mu)$
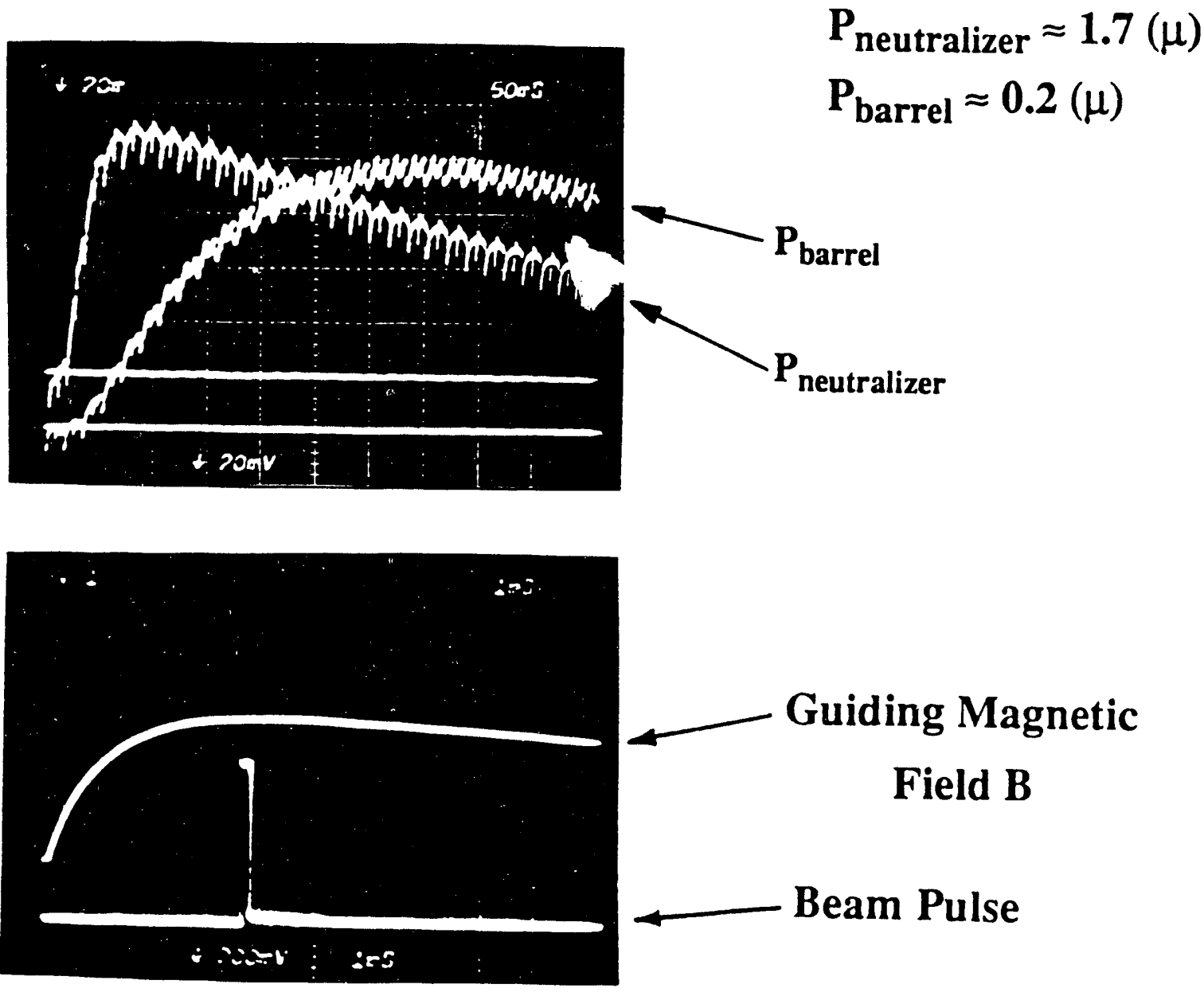

Guiding Magnetic

Field B

Beam Pulse

Figure 1.16 The timing sequency of the neutral hydrogen beam. 


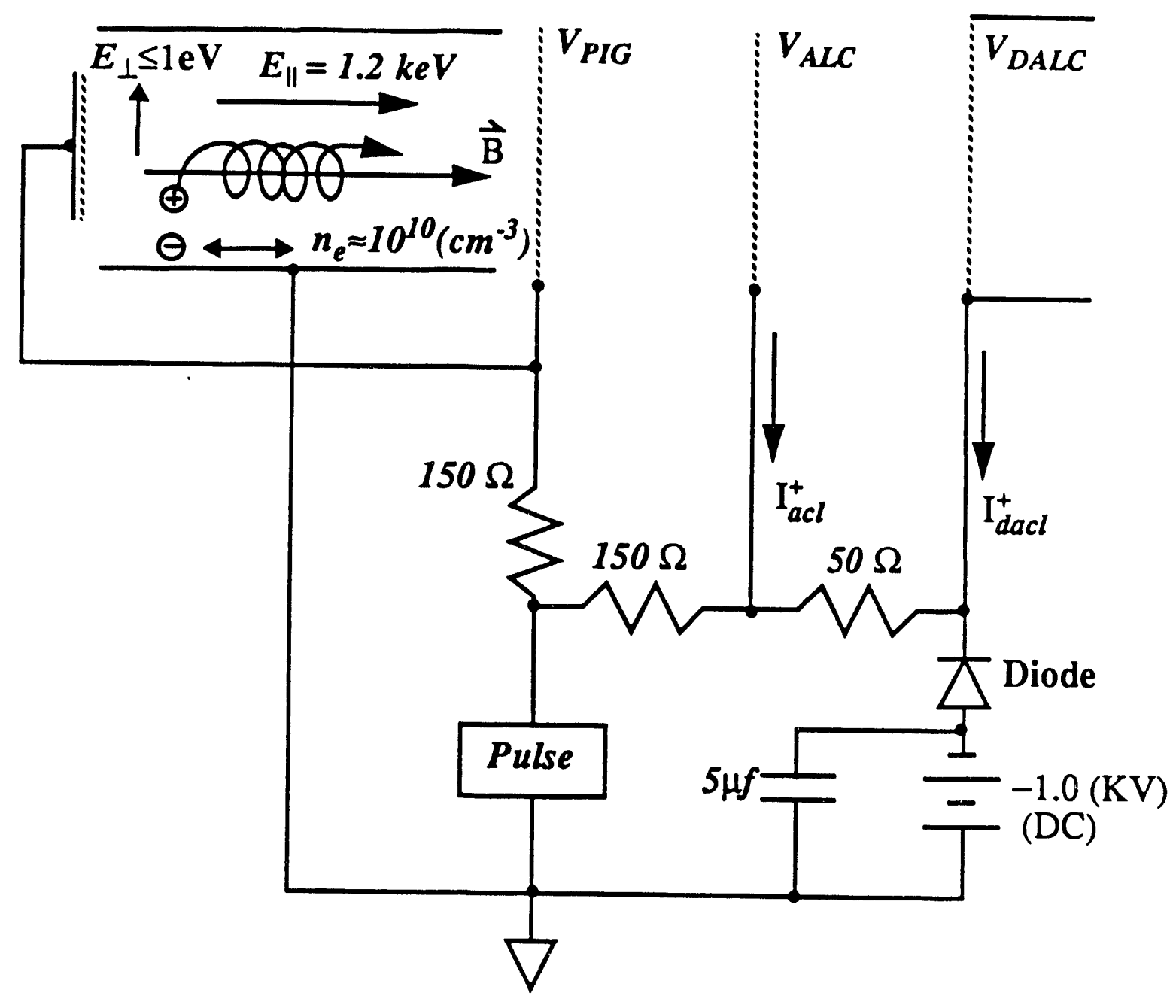

- The saturated ion flux which can be extracted from the source plasma:

$$
J_{i}=n_{i} e \sqrt{k T_{e} / m_{i}} \approx 0.1\left(A / \mathrm{cm}^{2}\right)
$$

Figure 1.17 The electric circuit of the discharge tube. 


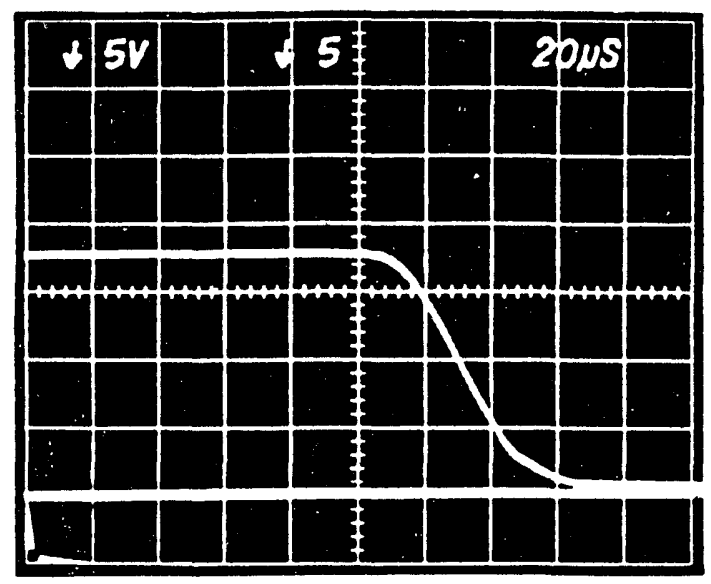

$$
\begin{aligned}
& \text { PIG - Tube: } \\
& \text { V }_{\text {PIG }}=-1.8(\mathrm{kV})
\end{aligned}
$$

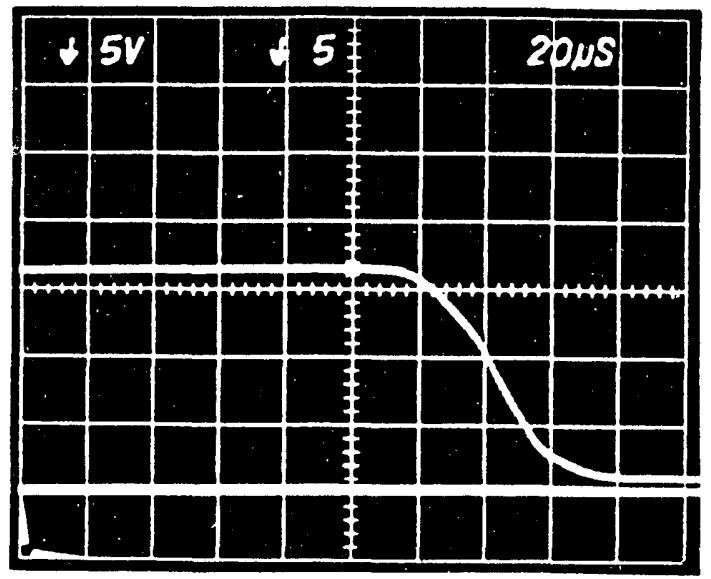

$$
\begin{aligned}
& \text { Accele. }- \text { Mesh: } \\
& V_{\text {ACL }}=-1.6(\mathrm{kV})
\end{aligned}
$$

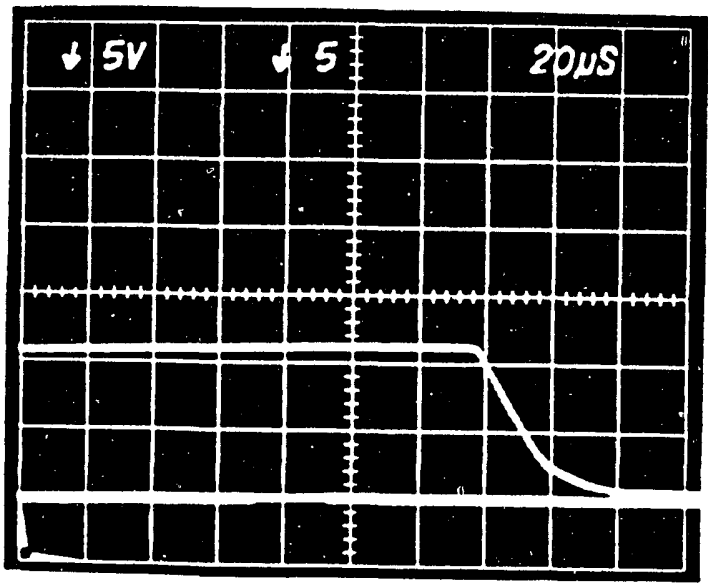

Deaccele. - Mesh:

$\mathrm{V}_{\text {DACL }}=-1.1(\mathrm{kV})$

Figure 1.18 The vacuum test of the bias voltage on the discharge tube, the accelerating and decelerating electrodes. 
(a)
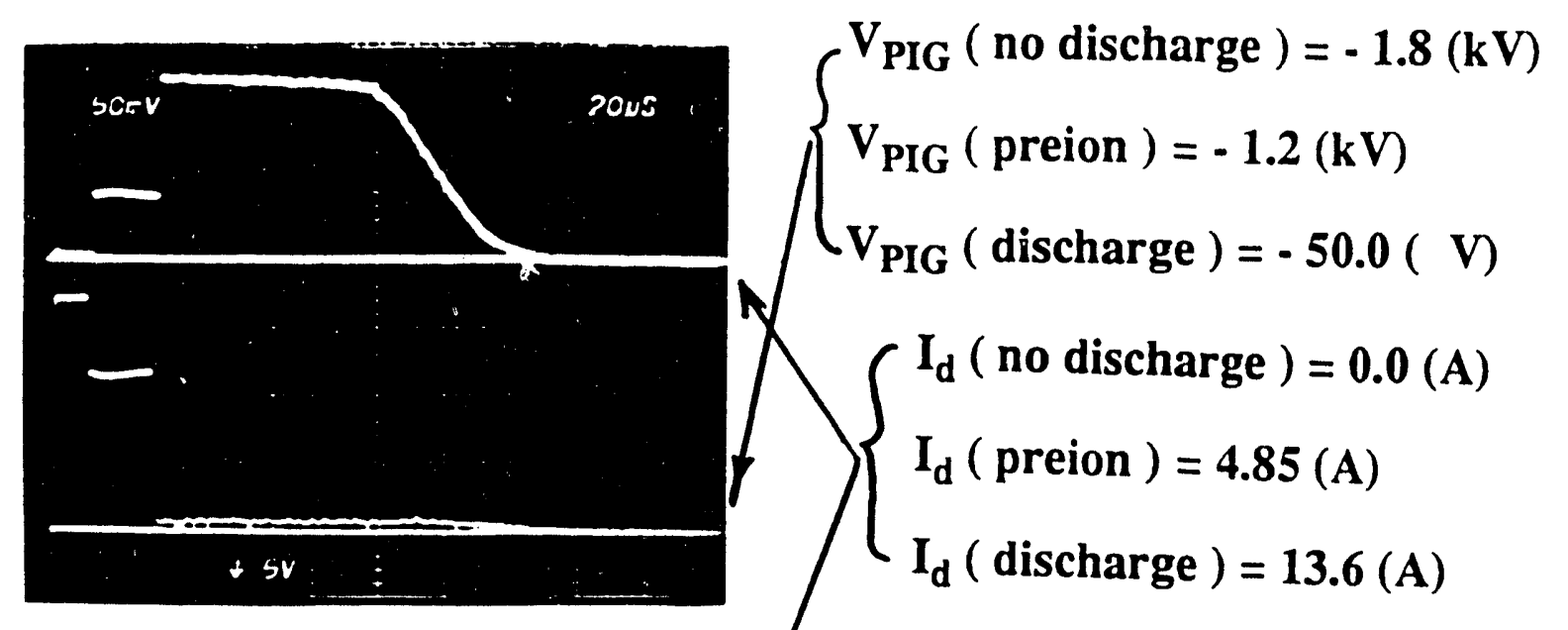

(b)
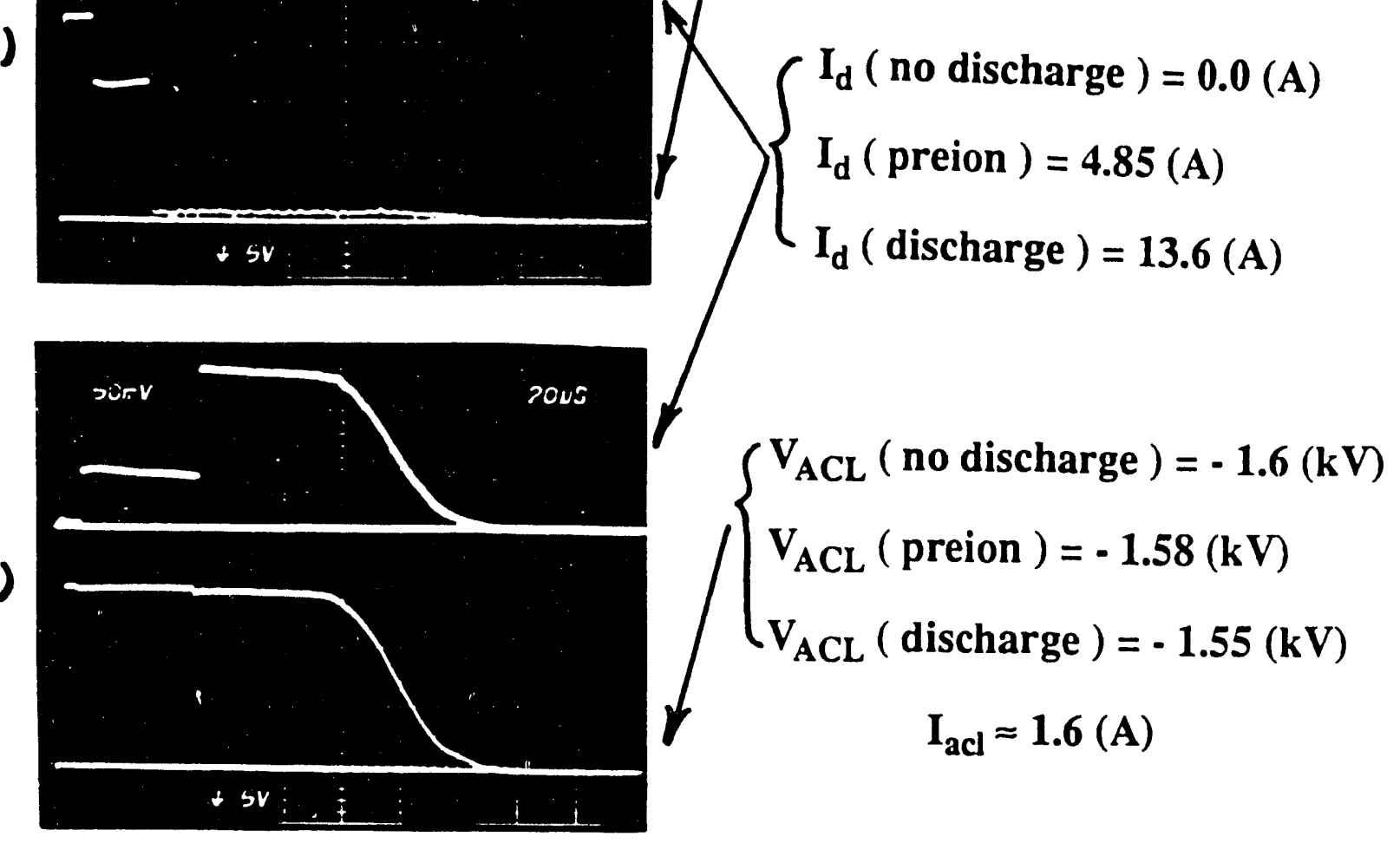

(c)

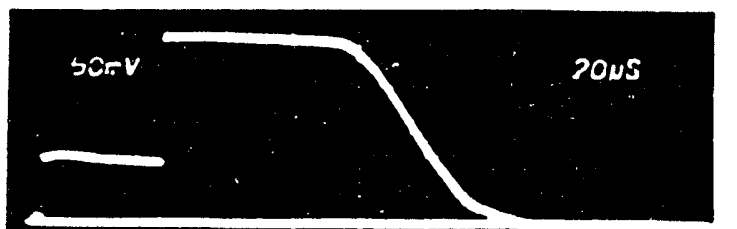

$I_{d}$

+ sv
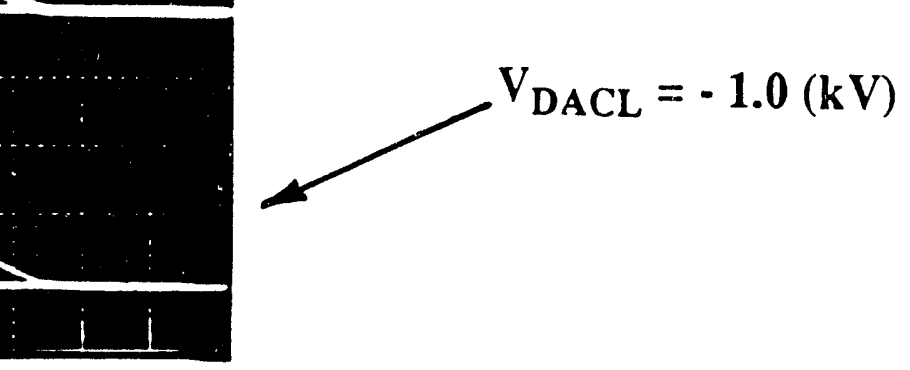

Figure 1.19 The discharge current and the bias voltage on the discharge tube, the accelerating and decelerating electrodes. 


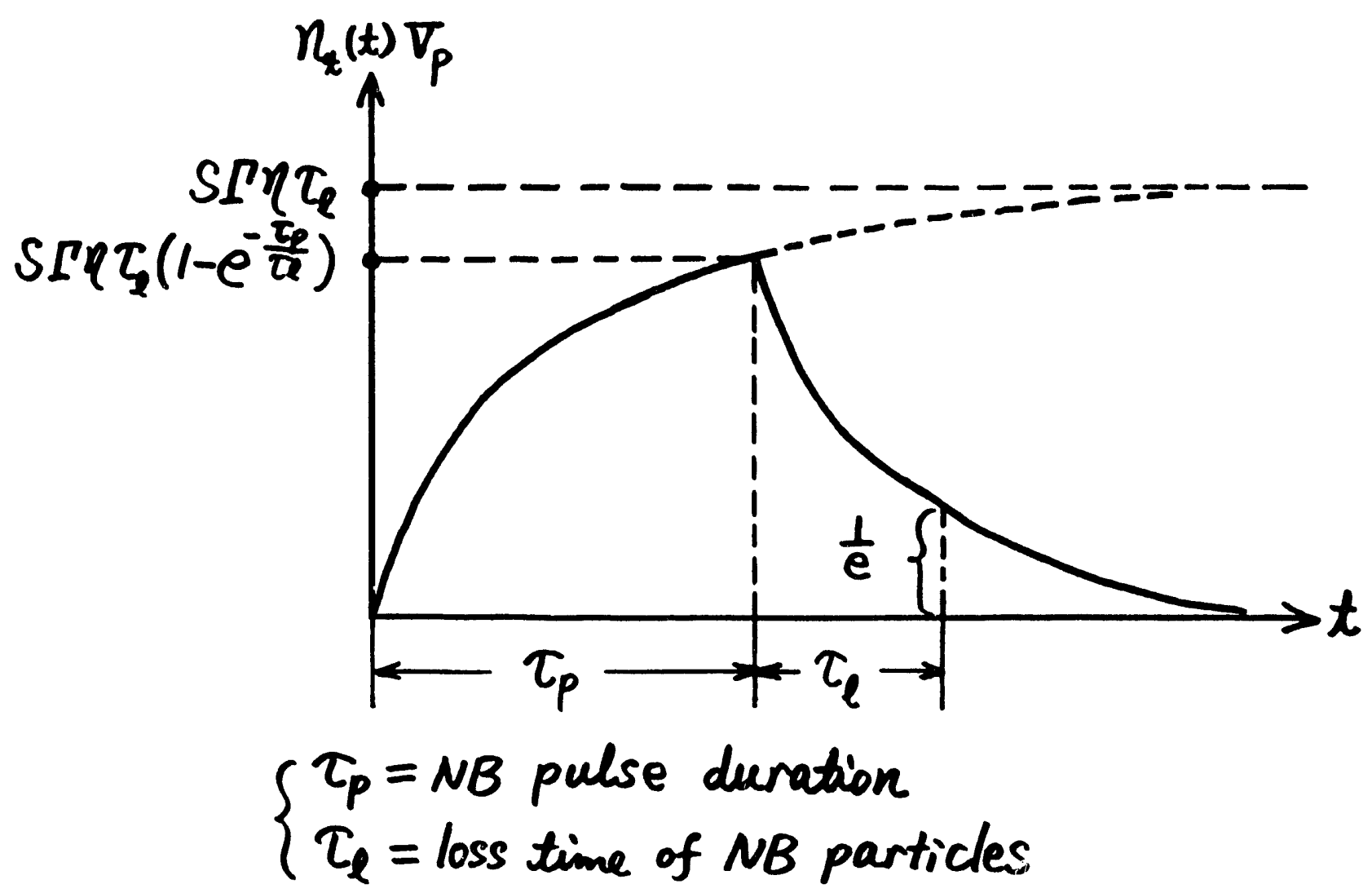

Figure 1.20 The confinement time of fast ion in HBT.

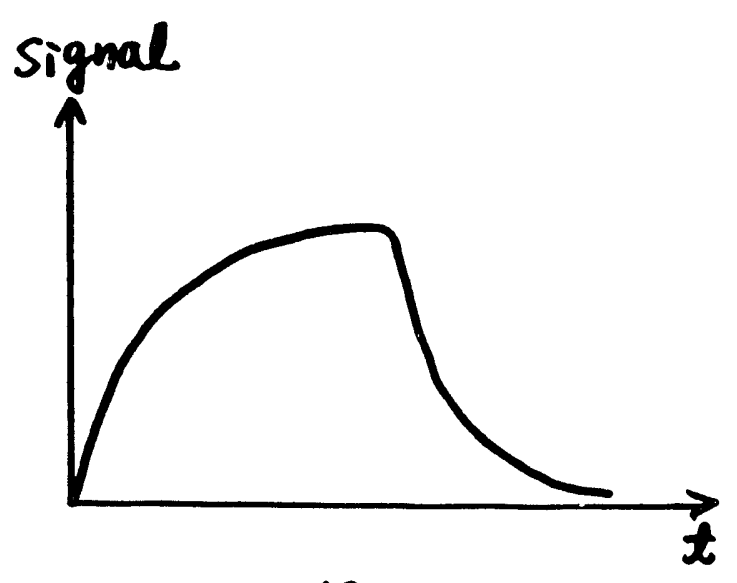

(a)

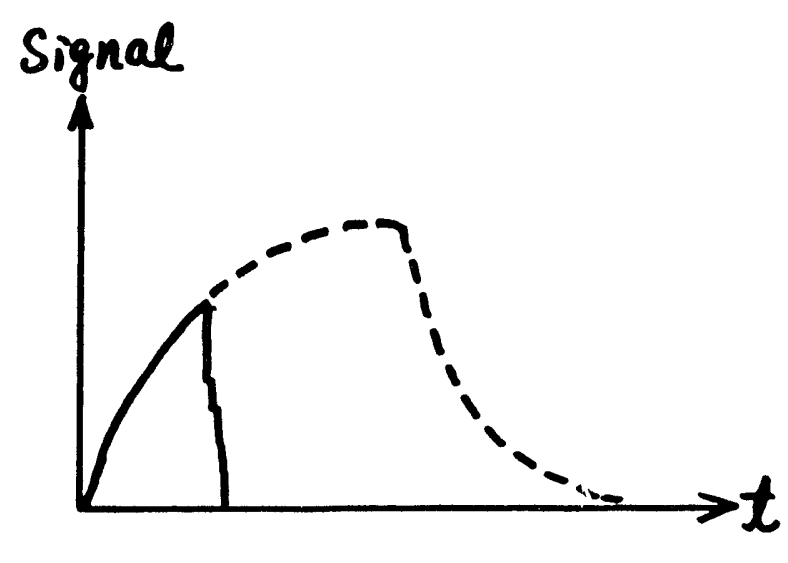

(b)

Figure 1.21 The detection of MHD instability: (a)when no instability happened; ( Wwhen an instability happened. 


\section{PART TWO:}

\section{ORBIT SIMULATION FOR NEUTRAL BEAM-INJECTED PARTICLES IN THE COLUMBIA HIGH BETA TOKAMAK (HBT)}

\subsection{Introduction}

A new diagnostic system, the neutral particle beam probe, has been suggested for the study of the magnetic field of the High beta Tokamak(HBT) $a-5.0 \mathrm{~cm}$, $T_{i} \approx T_{e} \leq 25(\mathrm{eV}), n_{e} \approx 5 \times 10^{14}\left(\mathrm{~cm}^{-3}\right)$ in Columbia University. The neutral beam energy is $1 \mathrm{keV}$, which is much higher than the plasma thermal energy. It will be used to test the containment of fast particles in the magnetic field structure of HBT under certain experimental conditions. The emission of the energetic particles from the plasma (due to losses which include MHD fluctuations that are too small or chaotic for a magnetic probe to detect) will be monitored by collectors located at the edge of the vacuum vessel. This note describes orbit calculations which show how the particles are contained within the plasma, subject to a number of perturbations in the time scale of thermalization of the fast ions which result from charge exchange in the fast neutrals.

Extensive numerical studies have been done to calculate the orbits of these fast ions. These simulations deal with several kinds of perturbations which may affect the 
containment of fast ions, such as the nonaxisymmetric toroidal field ripple due to the finite number and nonuniform winding of the toroidal field coil, the ringing of the toroidal magnetic field due to the self and mutual inductance in the firing circuit, and some maci scopic displacement of the plasma due to kink-type instabilities. Several injection geometries are also examined numerically, for example, co-injection, counter-injection, vertical injection, etc. Different initial conditions for the ionization of these fast neutral particles are also discussed for each injection case.

The theoretical model is formulated in section 2.2 , and the numerical method is briefly described in section 2.3 . Some simulation results are presented and discussed in section 2.4. A summary is given in section 2.5 .

\subsection{The Theoretical Model}

Once the neutral particle beam is injected into HBT, the energetic neutrals will experience many collisions with the background plasma ions, electrons, and some impurity ions, becoming ionized. Cross section data for NB injection have been published by Riviere in 1971 and some analytical expressions were formed which described the collision data reasonably well. When the beam energy is about $1 \mathrm{keV}$, charge exchange will be the dominant ionization process because of the resonant interactions among like particles. In a typical HBT operating condition, the 
characteristic mean free path for the ionizations of neutrals is a few centimeters and the beam particles can penetrate deep into the plasma. The fast ions, if they are well confined inside the plasma, will transfer their surplus energies to the plasma via a series of Coulomb collisions until they are thermalized. Two time scales are important here, the average thermalization time which is needed to slow the fast protons down to the plasma thermal energy, and the confinement time of the fast protons before being ejected due to some perturbation as mentioned in section 2.1 . For the HBT plasma the thermalization time is about 10 ( $\mu \mathrm{sec}$ ) according to the formulas discussed by, e.g. Menon, etc. So the remaining question is how long the fast ions can be confined, and if the containment time is comparable to the energy-loss time.

Since the injected particles have much higher energy $(\approx 1 \mathrm{keV})$ than the plasma thermal energy $(\approx 25 \mathrm{ev}$ ), each distant encounter has a little effect on beam particles, and a single particle model is used to describe the behavior of fast protons. The interactions among these fast protons are also neglected because the density of injected fast protons is much lower than the plasma density. The equation of motion for a fast proton is

$$
m \frac{\overrightarrow{d V}}{d t}=e(\vec{E}+\vec{V} \times \vec{B})
$$

where $\vec{E}$ is the electric field of the plasma, and $\vec{B}$ is the total magnetic field including 
not only toroidal and poloidal field $\vec{B}_{t}$ and $\vec{B}_{p}$, but also various kinds of perturbing fields. If we choose a toroidal coordinate system shown in Fig. $1, \vec{E}$ and $\vec{B}$ can be expressed as

$$
\left\{\begin{array}{l}
\vec{E}=E_{t} \hat{e}_{\phi}+E_{r} \hat{e}_{r}=E_{t} \hat{e}_{\phi}+E_{r} \cos \theta \hat{e}_{R}+E_{r} \sin \theta \hat{e}_{Z} \\
\vec{B}=B_{t} \hat{e}_{\phi}+B_{\theta} \hat{e}_{\theta}+B_{Z} \hat{e}_{Z}+B_{R} \hat{e}_{R}+B_{r} \hat{e}_{r}
\end{array}\right.
$$

Then the equation of motion can be written in component form as follows

$$
\left\{\begin{array}{l}
\frac{d R}{d t}=V_{R} \\
\frac{d Z}{d t}=V_{Z} \\
\frac{d \phi}{d t}=\frac{V_{\phi}}{R} \\
\frac{d V_{R}}{d t}=\frac{V_{\phi}^{2}}{R}+\frac{q}{m}\left[E_{r} \cos \theta+V_{Z} B_{t}-V_{\phi}\left(B_{Z}+\sin \theta B_{r}+\cos \theta B_{\theta}\right)\right] \\
\frac{d V_{Z}}{d t}=\frac{q}{m}\left[E_{r} \sin \theta-V_{R} B_{t}+V_{\phi}\left(B_{R}-\sin \theta B_{\theta}+\cos \theta B_{r}\right)\right] \\
\frac{d V_{\phi}}{d t}=-\frac{V_{R} V_{\phi}}{R}+\frac{q}{m}\left[E_{\phi}+V_{R} B_{Z}-V_{Z} B_{R}+B_{\theta}\left(\sin \theta V_{Z}+\cos \theta V_{R}\right)+B_{r}\left(\sin \theta V_{R}-\cos \theta V_{Z}\right)\right]
\end{array}\right.
$$

There are 6 unknowns $\left(R, Z, \phi, V_{R}, V_{Z}, V_{\phi}\right)$ and 6 equations, so the set is closed. Under a given initial condition, these equations describe a unique orbit curve of one proton in the 6-D phase space $(\vec{X}, \vec{V})$.

The toroidal component of the plasma electric field $E_{t}$ is obtained by using the relation $I_{p}^{2} R_{p}=E_{t} J_{t} V$, which describes the power dissipation into the plasma from the external field, where $V, I_{p}$ and $J_{t}$ are the total plasma volume, the toroidal plasma 
current and current density respectively. The plasma resistance $R_{p}$ is calculated using Spitzer's formula. The perpendicular component $E_{r}$ is estimated as $\left[\frac{k T_{e}}{a e}\right]$ which represents a balance of the electric and thermal energy in the minor radial direction. For a typical HBT operational condition ( $I_{p} \approx 20 \mathrm{kA}, T_{e} \approx 25 \mathrm{eV}$ and $a \approx 5.0 \mathrm{~cm}$ ), $E_{t}$ and $E_{r}$ are about $10.6 \mathrm{~V} / \mathrm{m}$ and $500.0 \mathrm{~V} / \mathrm{m}$, respectively. According to these numbers we can estimate that the electric force is much weaker $\left(10^{-3} \sim 10^{-4}\right.$ times lower $)$ than the magnetic force acting on a single fast ion. The simulation indeed also showed that the electric field has very little contribution to the fast proton orbit and can be neglected (see Fig. 2.6 for zero electric field and Fig. 2.11 for non-zero electric field cases).

The rippled toroidal vacuum magnetic field can be modeled as

$$
B_{t v}(R, \phi, t)=B_{\phi 0}\left[\frac{R_{0}}{R}\right][1-\delta(R) \cos (N \phi)]\left[1+\eta e^{-\gamma t} \sin (\omega t)\right],
$$

where $N=4$ is the number of winding of the toroidal coil. The ripple $\delta$, in general, is a function of not only the main radius $R$, but also the poloidal angle $\theta$, and is very difficult to express in an analytical form. We first calculate the current distribution within each group of coil set using a code, namely "COILS", for a given total current (see Fig. 2.2) considering the self and mutual inductance of the coil system. Then we 
calculate the magnetic field $\vec{B}$ using"EFFI" code, and got the ripple $\delta(R)$ as shown in Fig. 2.3. The B-contours and A-contours of the toroidal magnetic field are plotted in Fig. 2.4 according to the current distribution in each coil as shown in Fig.2.2. As a comparison Fig. 2.5 is the contour plot of toroidal magnetic field resulting from a uniformly current distribution. Obviously, the flat current profile in the coil system gives a higher ripple level than that of self-consistent current profile from COILS code. The factor $\eta(\approx 10 \%)$ is used to model the relative level of the ringing of the magnetic field at frequency $\omega \approx 1.57 \mathrm{MHZ}$, which decays in about $\frac{1}{\gamma} \approx 10 \mu \mathrm{sec}$.

The required vertical field for the equilibrium in the main radial direction can be calculated from the force balance equation, assuming a circular cross-section plasma and neglecting any toroidal rotation, and is expressed as

$$
B_{\text {vertical }}=\frac{\mu_{0} I_{p}}{4 \pi R_{0}}\left[\ln \left[\frac{8 R_{0}}{a}\right]-1+\frac{1}{2}\left(l_{i}+\beta_{p}+\mu_{i}\right)\right] \text {, }
$$

where $\beta_{p}$ is the poloidal beta $(\approx 4 \%), I_{p}$ the toroidal plasma current, $l_{i}$ the internal inductance per unit length of the plasma $(\approx 0.7), \mu_{i}$ the diamagnetism of plasma $(=4)$. It can be calculated that $B_{\text {vertical }}$ is 583 (Gauss) for the plasma current $I_{p}=20(k A)$. The vertical field $B_{\text {vertical }}$ is externally imposed and is independent of the major radius. 
The main radial component(in $\hat{e}_{R}$-direction) of the vacuum magnetic field, which results from the ripple, is obtained from $\nabla \cdot \vec{B}=0$ by using equations (2.4) and (2.5), and can be written in the form as

$$
B_{\text {ripple }}(R, \phi)=\frac{R_{0}}{R}\left[B_{R_{0}}-B_{\phi_{0}} N \sin (N \phi) \int_{R_{0}}^{R} \frac{\delta(R)}{R} d R\right] \text {, }
$$

where $B_{R_{0}}=B_{R}\left(R_{0}, \phi\right)$. The ripple profile is quite flat (see Fig.2.3) and is very small at the vicinity of $R=R_{0}$, so it is a good approximation to choose $\delta(R)=\delta_{0}=$ constant. Then $B_{\text {ripple }}$ can be expressed as

$$
B_{\text {ripple }}(R, \phi)=\frac{R_{0}}{R}\left[B_{R_{0}}+B_{\phi_{0}} N \delta_{0} \ln \left[\frac{R_{0}}{R}\right] \sin (N \phi)\right]
$$

The poloidal field is simply calculated from Ampere's law assuming a circular cross-section plasma and a uniform current profile, and in the form as

$$
B_{\theta p}=B_{\theta_{0}} \begin{cases}{\left[\frac{r}{a}\right]} & (0 \leq r \leq a) \\ {\left[\frac{a}{r}\right]} & (a \leq r<b),\end{cases}
$$

where $r=b$ is the wall of the vacuum vessel.

In order to get the analytical expression of a kink perturbation in magnetic field, we started from the energy principle. For the case of internal kink modes, the perturbation is constrained to the helical form and the displacement can be written as 


$$
\vec{\xi}(r, \theta, z)=\vec{\xi}(r) e^{i\left(m \theta+k_{z} Z\right)}=\vec{\xi}(r) e^{i(m \theta+n \phi)}
$$

Consider the special case of $m=1$ and $n=1$ i.e. $k_{z} Z=\phi$ where $\phi$ is the toroidal angle.

Under the limit $k_{z}, Z \ll 1$, the potential energy for the perturbation can be minimized by the singular function $\xi(r)$ which is defined as follow

$$
\xi(r)= \begin{cases}\xi_{a} & (r<s) \\ 0 & (r>s)\end{cases}
$$

where $s$ is the singular surface at which $\vec{k} \cdot \vec{B}=k_{z} B_{z}+\frac{B_{p}}{r}=0$. This is also equivalent to $q(r=s)=1$. Then the kink fluctuating magnetic field can be derived from the relation

$$
\vec{B}_{k}=\nabla \times(\vec{\xi} \times \vec{B})
$$

and is approximately expressed as follows:

$$
\left\{\begin{array}{l}
B_{k r}=-\xi_{a} \sin (\theta+\phi)\left(\frac{B_{p}(r)}{r}+\frac{B_{t}(R)}{R}\right) \\
B_{k \theta}=-\xi_{a} \cos (\theta+\phi)\left(\frac{\partial B_{p}(r)}{\partial r}+\frac{B_{t}(R)}{R}\right) \\
B_{k c}=B_{k \phi}=\xi_{a} \cos \phi \frac{B_{t}(R)}{R},
\end{array}\right.
$$

where $B_{t}=B_{t 0}\left[\frac{R_{0}}{R}\right]$ is the toroidal vacuum field. The poloidal magnetic field $B_{p}(r)$ is given in equation (2.8). The singular surface $r=s$ is estimated in the following way: we wrote $s=a+\Delta r$ where $\Delta r$ is the distance betwecn the singular surface and the 
plasma boundary $(a=5.0 \mathrm{~cm})$, and simulated $\Delta r$ for different values (e.g., $-1.5,-1.0$, $-0.5,0.0,0.5,1.0,1.5 \mathrm{~cm})$. The results did not show a strong effect on the confinement of fast protons; so we choose $s=a$, i.e. on the plasma surface. The perturbation amplitude $\xi_{a}$ is also simulated from 0.1 to $3.0 \mathrm{~cm}$. The magnetic probe data of plasma magnetic center showed that the kink-type instabilities indeed did shift the plasma a few centimeters away from its equilibrium position. So far we have discussed the analytical forms for each component of the magnetic field in equation (2.2). Let us write them in the following way for clarity:

$$
\left\{\begin{array}{l}
B_{t}=B_{t v}+B_{k \phi} \\
B_{\theta}=B_{\theta p}+B_{k \theta} \\
B_{Z}=B_{v e r t i c a l} \\
B_{R}=B_{r i p p l e} \\
B_{r}=B_{k r}
\end{array}\right.
$$

where subscript $v$ denotes the vacuum field.

Three kinds of plasma current profile are used in these simulations to check the fast ion containment under different plasma current profile. These profiles are:

the constant current profile:

$$
J(r)= \begin{cases}J_{0}=\text { constant } & (0 \leq r \leq a) \\ 0 & (a<r \leq b)\end{cases}
$$


the parabolic current profile:

$$
J(r)= \begin{cases}J_{0}\left[1-\left[\frac{r}{a}\right]^{2}\right] & (0 \leq r \leq a), \\ 0 & (a<r \leq b) ;\end{cases}
$$

and the hollow current profile:

$$
J(r)= \begin{cases}J_{0}\left[\frac{r}{a}\right] & (0 \leq r \leq a), \\ 0 & (a<r \leq b) .\end{cases}
$$

\subsection{Numerical Method}

In these simulations we define some 6-D vectors as follows.

$$
\vec{y}=\left(\begin{array}{l}
y(1) \\
y(2) \\
y(3) \\
y(4) \\
y(5) \\
y(6)
\end{array}\right)=\left(\begin{array}{l}
R \\
z \\
\phi \\
V_{R} \\
V_{Z} \\
V_{\phi}
\end{array}\right), \vec{S}=\vec{y}(t=0)=\left(\begin{array}{c}
R_{0}+\Delta \\
Z_{0} \\
\phi_{0} \\
-V_{\perp 0} \\
V_{Z 0} \\
\pm V_{\| 0}
\end{array}\right)
$$

where $V_{\perp 0}, \pm V_{\| 0}$ and $V_{Z 0}$ are the $\hat{e}_{R}-, \hat{e}_{\phi}-$ and $\hat{e}_{Z}$-components of the initial velocity, respectively, and the \pm sign corresponds to the co- and counter-injection case, $\Delta=R(t=0)-R_{0}$ is the initial ionization position of the injected neutral particles.

Equation (2.3) can also be written in the vector form

$$
\left\{\begin{array}{l}
\vec{y}(t)= \begin{cases}\vec{f}(t, \vec{y}) & (0 \leq r \leq a) \\
\vec{g}(t, \vec{y}) & (a \leq r \leq b)\end{cases} \\
\vec{y}(t=0)=\vec{S},
\end{array}\right.
$$


where $\vec{f}$ and $\vec{g}$ are the functions on the right hand side of equation (2.3). The RungaKutta method gives the following iteration formula

$$
\begin{gathered}
y_{n+1}(i)=y_{n}(i)+\frac{h}{6}\left[Q_{n}(i, 1)+2\left(Q_{n}(i, 2)+Q_{n}(i, 3)\right)+\right. \\
\left.Q_{n}(i, 4)\right](i=1,2, \cdots, 6),
\end{gathered}
$$

where $Q_{n}{ }^{\prime} s$ are the $f$ and $g$ related functions.

For a $1 \mathrm{keV}$ neutral hydrogen beam, $V_{\perp 0}=4.4 \times 10^{7} \mathrm{~cm} / \mathrm{sec}$, the gyroradius $r_{c}=$ $4.0 \mathrm{~cm}$ at $B=2.0 \mathrm{kG}$, then the gyroperiod $T_{c}$ is $0.1 \mu \mathrm{sec}$. The time step $h$ for the iteration is chosen as $\frac{T_{c}}{100}=1.0 \mathrm{nsec}$. For example, the $50 \mu \mathrm{sec}$ time interval of the orbit simulation will take 50,000 steps of iteration; the Cray computer can easily finish it in a very short time.

\subsection{Simulation Result}

The simulation shows that fast proton trajectories depend on the injection geometry, plasma current, and the perturbations of the magnetic field. The injection geometry is partially tangential (in the $\hat{e}_{\phi}$-direction) and partially perpendicular( in the $\hat{e}_{R}$-direction). The injection angle $\theta$ is defined as the angle between the beam and the $\hat{e}_{R}$ - directions. In our experiment, the maximum injection angle is about $14^{\circ}$, i.e. the initial pitch angle $\chi(t=0)=\cos ^{-1}\left(\frac{V_{\|}}{V}\right)=76^{\circ}$. The plasma current is chosen in 
the $\hat{e}_{\phi}$-direction. The term "co- or counter-"injection means that the tangential component of the velocity is parallel, $(\vec{V} \cdot \vec{J}>0)$ or anti-parallel $(\vec{V} \cdot \vec{J}<0)$ to the direction of plasma current. Two plots are generated for each case to show the orbit: the toroidal projection(for $R$ and $\phi$ ) on the $Z=0$ plane and the poloidal projection(for $R$ and $Z$ ) on the $\phi=0$ plane.

\subsubsection{Ideal Case (i.e. no ripple, ringing, kink perturbations, etc.)}

Both toroidal and poloidal projections of the fast proton orbit tell us that there are two kinds of orbits: trapped and untrapped. The trapped protons mirror in the $B_{\phi}=B_{\phi 0}\left[\frac{R_{0}}{R}\right]$ toroidal field and the poloidal projection of the guiding centers are "banana" shapes. They are mainly confined to the outside of the minor axis, and eventually shift outward and lost to the walls without significantly transferring their energy to the plasma. The untrapped proton can travel along both the major and the minor axes and the poloidal projection of the guiding center is a shifted circle. The results also showed the dependence on the initial ionization position and the ratio of $V_{\|}$to $V_{\downarrow}$ for the fast proton can produce two different kinds of orbit. The larger the ratio of $V_{\|}$ to $V_{\perp}$ (i.e., the larger the pitch angle), the m(re untrapped orbits. When the ionization position is more inward away from the minor axis, more untrapped orbits are pro- 
duced. Fig.1.3 and Fig.1.4 show the calculated neutral beam flux profile which is attenuated as the penetration distance increased. For the case of injection of neutral hydrogen beam into hydrogen plasma, most particles are ionized before crossing the magnetic axis because the strong resonant interaction between the beam and the field particles. But for the helium plasma, about $30 \%$ of the beam can penetrate through it because of the weak interactions between the unlike particles.

Another result is that there is a significant difference in the orbit formation between the co- and counter-injection. Co-injection is preferred because it yields more orbits remaining confined to the plasma volume. This can also be simply proved from the analysis of dynamical equilibrium between the centrifugal force $m \frac{V_{\phi}^{2}}{R}$ and the centripetal force $e \vec{V}_{\phi} \times \vec{B}_{\theta}$ due to the poloidal magnetic field.

The plots shown from Fig.2.7 to Fig.2.16 are the simulation results for both coand counter-injection cases according to the three kinds of plasma current profile as mentioned before, under the different initial ionization locations.

\subsubsection{Ripple Field Effect}

The nonaxisymmetric ripple field can form local mirror fields and particles will be trapped there and eventually drift out. The ripple effect is stronger in the outside 
region of the vacuum vessel at the relatively large separations among coils. In our time scale of interest $(\approx 30 \mu \mathrm{sec}), 2 \sim 3 \%$ ripple fields has little influence on the particle orbit confinement. But when the ripple level is over $4 \sim 5 \%$, fast protons will be dumped out in less than about $5 \sim 10 \mu \mathrm{sec}$ ), see Fig.2.17 and Fig.2.18.

\subsubsection{The Ringing Effect Of The Toroidal Field}

The fast ringing $\left(\omega_{r} \approx 1.6 \mathrm{MH}_{Z}\right)$ of the toroidal magnetic field has almost no effect on the orbit, comparing Fig.2.11 and Fig.2.19. The gyroperiod $T_{g}(\approx 0.1 \mu \mathrm{sec})$ is much smaller than the ringing period $T_{r}(\approx 4.0 \mu \mathrm{sec})$, so the fast ringing is still a slow variation in one gyration. Also note that the ringing is damped in about $10 \mu \mathrm{sec}$ and the neutral particle beam can be injected after that.

\subsubsection{The Kink Effect}

The simulation shows that this is the worst perturbation for the particle orbit confinement. Under this condition, the energetic protons are indeed ejected before they cool down, but only for rather vigorous kinking (when $\xi_{a} \geq 1.0 \mathrm{~cm}$ ) as shown from Fig.2.20 to Fig.2.22. The containment time of the particle orbit is only about $5-10 \mu s e c$, depending on the perturbation amplitude $\xi_{a}$ and the initial ionization position $r_{i}$. The plots of pitch angle also display an abnormal change when the kinking 
happened.

\subsubsection{Vertical Injection}

Vertical injections from the bottom of HBT are also simulated and the similar results are obtained as that of horizontal injection. Fig.2.23 is one of the many simulations from vertical injection geometry. Comparing this with Fig.2.11 we can see that for parabolic current profile the containment is better than that of horizontal injection, but worse for the case of constant and hollow current profile.

\subsubsection{Global Parameter Variation}

Many parameters as shown in Fig.2.24 are examined during each calculation to diagnose the simulation process, such as the total kinetic energy, the magnetic moment, the pitch angle, the radial and vertical displacement and the toroidal canonical momentum which will be discussed in next section. When the electric field was chosen to be zero the total kinetic energy from the simulation output is exactly a constant.

\subsubsection{Other Simulations}

The fast ion orbit was also calculated by using the magnetic field obtained from a 2-D MHD code which was developed by Dr. X.L. Li at Columbia Plasma Physics 
Lab. This code outputs the magnetic field components $B_{r}, B_{Z}$, the magnetic vector potential $\psi$, etc. at each grid point. We use $R_{i}$ and $Z_{j}$ to denote the grid (or lattice) coordinate and $R^{k}$ and $Z^{k}$ for the coordinate of particle orbit, and write:

$$
\left\{\begin{array}{l}
B_{R}\left(R_{i}, Z_{j}\right)=B_{R_{i, j}}, \\
B_{Z}\left(R_{i}, Z_{j}\right)=B_{Z_{i, j}}, \\
B_{\phi}\left(R_{i}, Z_{j}\right)=B_{\phi_{i, j}}
\end{array}\right.
$$

Then we use Taylor interpolation to derive the related quantities at an arbitrary point $k$, as shown in Fig.2.25, as follows:

$$
\left\{\begin{array}{l}
B_{R}^{k}=B_{R E}+\frac{R^{k}-R_{i}}{\Delta R}\left(B_{R F}-B_{R E}\right) \\
B_{Z}^{k}=B_{Z M}+\frac{Z^{k}-Z_{j}}{\Delta Z}\left(B_{Z N}-B_{Z M}\right) \\
B_{\phi}^{k}=\frac{B_{\phi E F}+B_{\phi M N}}{2.0} \\
B^{k}=\left[\left(B_{R}^{k}\right)^{2}+\left(B_{Z}^{k}\right)^{2}+\left(B_{\phi}^{k}\right)^{2}\right]^{1 / 2}
\end{array}\right.
$$

where the related quantities in equation (2.21) are defined as follows:

$$
\left\{\begin{array}{l}
B_{\phi E F}=B_{\phi E}+\frac{R^{k}-R_{i}}{\Delta R}\left(B_{\phi F}-B_{\phi E}\right) \\
B_{\phi M N}=B_{\phi M}+\frac{Z^{k}-Z_{j}}{\Delta Z}\left(B_{\phi N}-B_{\phi M}\right)
\end{array}\right.
$$

and 


$$
\left\{\begin{array}{l}
B_{R E}=B_{R_{i, j}}+\frac{Z^{k}-Z_{j}}{\Delta Z}\left(B_{R_{i, j+1}}-B_{R_{i, j}}\right), \\
B_{R F}=B_{R_{i+1, j}}+\frac{Z^{k}-Z_{j}}{\Delta Z}\left(B_{R_{i+1, j+1}}-B_{R_{i+1, j}}\right) \\
B_{Z M}=B_{Z_{i, j}}+\frac{R^{k}-R_{i}}{\Delta R}\left(B_{Z_{i+1, j}}-B_{Z_{i, j}}\right) \\
B_{Z N}=B_{Z_{i, j+1}}+\frac{R^{k}-R_{i}}{\Delta R}\left(B_{Z_{i+1, j+1}}-B_{Z_{i, j+1}}\right)
\end{array}\right.
$$

In Fig.2.26, the simulation results were obtained at three different initial ionization locations. The fast ions are also well contained in these kind of magnetic field configuration for at least $25 \mu \mathrm{sec}$. Fig. 2.27 is another calculation with the plot of toroidal canonical momentum, $P_{\phi}$, defined as:

$$
\begin{aligned}
P_{\phi} & =m R^{2} \dot{\phi}+\frac{q}{c} A_{\phi} R \\
& =m R(R \dot{\phi})+\frac{q}{c}\left[\frac{\psi}{R}\right] R \\
& =m R V_{\phi}+\frac{q}{c} \Psi, \quad\left(g \mathrm{~cm}^{2} / \mathrm{sec}\right) .
\end{aligned}
$$

Since the 2-D MHD code assumes a toroidal symmetry, i.e. $\frac{\partial}{\partial \phi}=0$, therefore $P_{\phi}$ should be a constant. From Fig.2.27 we can see that $P_{\phi}$ is nearly, but not strictly, a constant. The little periodical variation may be caused by some numerical error accumulation when we interpolate the related quantities in an arbitrary orbit location. 


\subsection{Summary}

Numerical studies show us that energetic $(\approx 1 \mathrm{keV})$ protons will be contained inside the HBT plasma in vacuum magnetic fields (including $\approx 2 \%$ ripple and small kinking displacement $\xi_{a}<1.0 \mathrm{~cm}$ ) for a time $\approx 30 \sim 35 \mu \mathrm{sec}$ which is at least comparable to the energy-loss time $(\approx 10 \mu \mathrm{sec})$.

Generally speaking, the constant plasma current profile provides the best containment of the fast ions and the hollow current profile plasma will more quickly eject these fast ions out of the plasma volume.

When the plasma undergues a large kink-type perturbation $\left(\xi_{a} \geq 1.0 \mathrm{~cm}\right)$, the energetic protons will be indeed ejected within $5 \sim 10 \mu \mathrm{sec}$ before being thermalized. Therefore, together with the additional task of developing a sensitive detactor for the ejected energetic particles, this suggests that the initial application of the NB diagnostic could be a "shine-through" experiment: a beam of neutrals is directed transverse to the plasma cross section, and a detector oppositely located measures the attenuation, given assumptions on the temperature profile (ohmic heating) and density profile $\left(\mathrm{CO}_{2}\right.$ interferometer). 


\section{Acknowledgements:}

We sincerely thank Dr. G. A. Navratil and Dr. M. E. Mauel for their valuable discussions.

This work was supported by U.S. Department of Energy contract No. DE-FG0286ER53222.

\section{References:}

[1] Riviere, A. C., "Penetration of Fast Hydrogen Atoms Into a Fusion Reactor Plasma", Nuclear Fusion 11, 1971, p.363-369.

[2] Sweetman, D. R., "Ignition Condition in Tokamak Experiments and Role of Neutral Injection Heating", Nuclear Fusion 13, 1973, p.157-165.

[3] Rome, J. A., Callen, J. D. and Clarke, J. F., "Neutral-Beam Injection into a Tokamak: Part I: Fast-Ion Spatial Distribution for Tangential Injection", Nuclear Fusion 14, 1974, p.141-151.

[4] Rome, J. A., McAlees, D. G., Callen, J. D. and Fowler, R. H., "Particle-Orbit Loss Regions and Their Effects on Neutral-Injection Heating in Axisymmetric Tokamaks", Nuclear Fusion 16, 1976, p.55-66. 
[5] Jassby, D. L., "Neutral-Beam-Driven Tokamak Fusion Reactors", Nuclear Fusion 172, 1977, p.309-365.

[6] Menon, M. M., "Neutral Beam Heating Applications and Development", Proceedings of IEEE, Vol. 69, No. 8, August 1981, p.1012-1029.

[7] Boozer, A. H., "Enhanced Transport in Tokamaks due to Toroidal Ripple", Physics of Fluids, Vol. 23, No. 11, November 1980, p.2283-2290.

[8] Boozer, A. H., "Guiding Center Drift Equations", Physics of Fluids, Vol. 23, No. 5, May 1980, p.904-908.

[9] Goldston, R. J. and Towner, H. H., "Effects of Toroidal Field Ripple on Suprathermal Ions in Tokamak Plasmas", J. Plasma Physics, Vol. 26, Part 2, 1981, p.283-307.

[10] Goldston, R. J., White, R. B. and Boozer, A. H., "Confinement of High-Energy Trapped Particles in Tokamaks", Physical Review Letters, Vol. 47, No. 9, August 1981, p.647-649.

[11] Linsker, R. and Boozer, A. H., "Banana Drift Transport in Tokamaks with Ripple", Physics of Fluids, Vol. 25, No. 1, January 1982, p.143-147.

[12] Mynick, H. E. and Hitchon, W. N. G., "Tokamak Superbanana Transport - 
Revisited", Nuclear Fusion, Vol. 24, No. 3, 1984, p.317-327.

[13] Mauel, M. E., Kink Oscillations Change Millimeter Wave Polarization During Tokamak Discharges, B.S. Dissertation, MIT, January 1978.

[14] Rosenbluth, M. N., Dagazian, R. Y. and Rutherford, P. H., "Nonlinear Properties of the Internal m=1 Kink Instability in the Cylindrical Tokamak", Physics of Fluids, Vol. 16, No. 11, November 1973, p.1894-1902.

[15] Zweben, S. J., "Pitch Angle Resolved Measurements of Escaping Charged Fusion Products in TFTR", PPPL-2582, January 1989.

[16] Shafranov, V. D., "Hydromagnetic Stability of a Current-Carrying Pinch in a Strong Longitudinal Magnetic Field", Soviet Physics-Technical Physics, Vol. 15, No. 2, August, 1970, p.175. (Translated from Zhurnal Teknichesheskoi Fiziki, Vol. 40, No. 2, February, 1970, p.241-253.

[17] Bussac, M. N., Pellat, R., Edery, D. and Soule, J. L., "Internal Kink Modes in Toroidal Plasmas with Circular Cross Sections", Physical Review Letters, Vol. 35, No. 24, December 1975, p.1638.

[18] Wesson, J. A., "Hydromagnetic Stability of Tokamaks", Nuclear Fusion, Vol. 18, 1978, p. 87. 


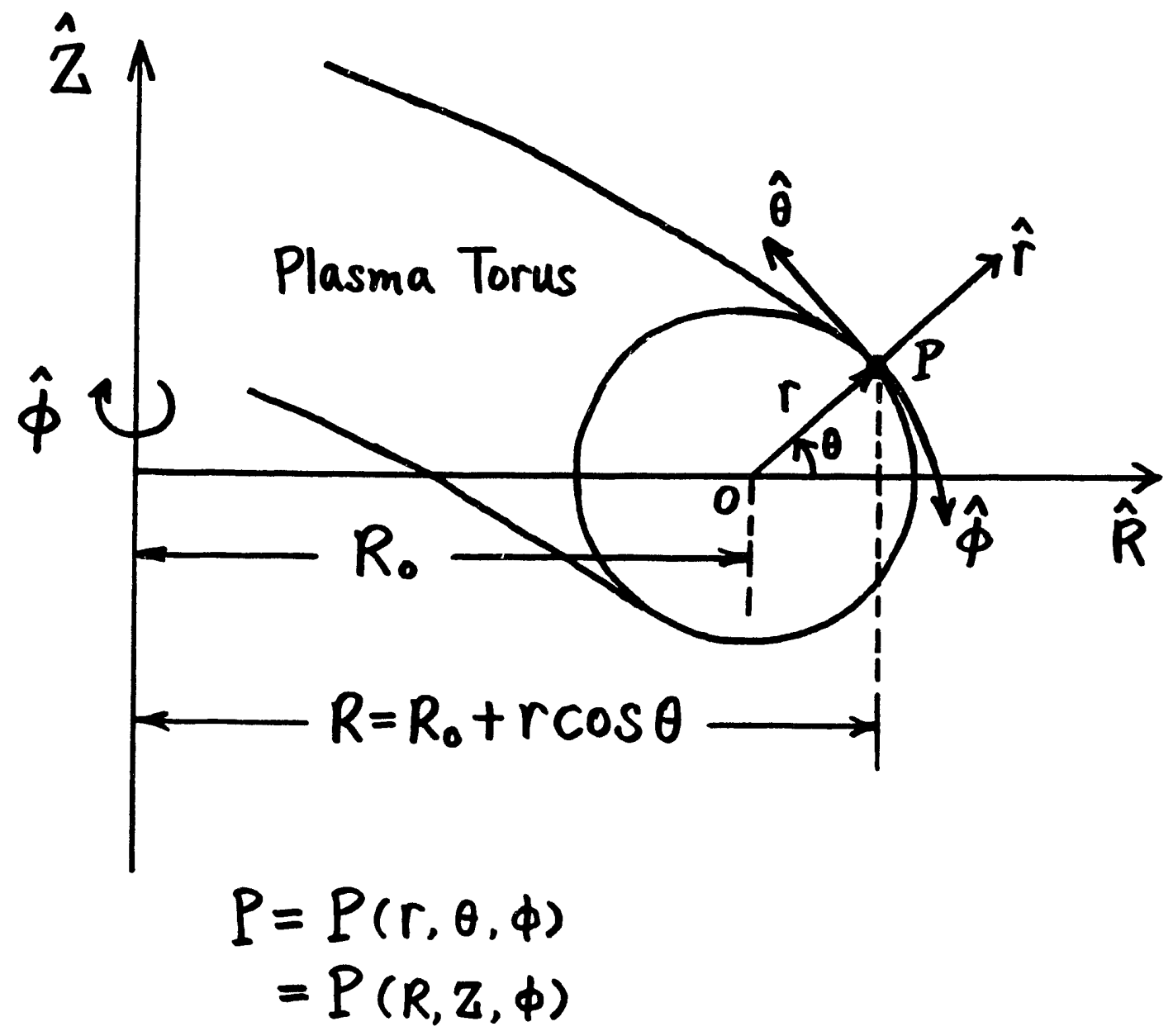

$$
\left\{\begin{array}{l}
R=R_{0}+r \cos \theta \\
Z=r \sin \theta \\
\phi=\phi
\end{array}\right.
$$$$
\left\{\begin{array}{l}
r=\sqrt{\left(R-R_{0}\right)^{2}+Z^{2}} \\
\theta=\sin ^{-1}\left(\frac{Z}{r}\right)=\cos ^{-1}\left(\frac{R-R_{0}}{r}\right) \\
\phi=\phi
\end{array}\right.
$$$$
\left\{\begin{array}{l}
\hat{e}_{r}=\cos \theta \hat{e}_{R}+\sin \theta \hat{e}_{Z} \\
\hat{e}_{\theta}=-\sin \theta \hat{e}_{R}+\cos \theta \hat{e}_{Z} \\
\hat{e}_{\phi}=\hat{e}_{\phi}
\end{array}\right.
$$

Figure 2.1 The coordinate system used in the calculation of fast ion orbit. 


\section{CURRENT OISTRIBUTION OF MAXWELL COIL}

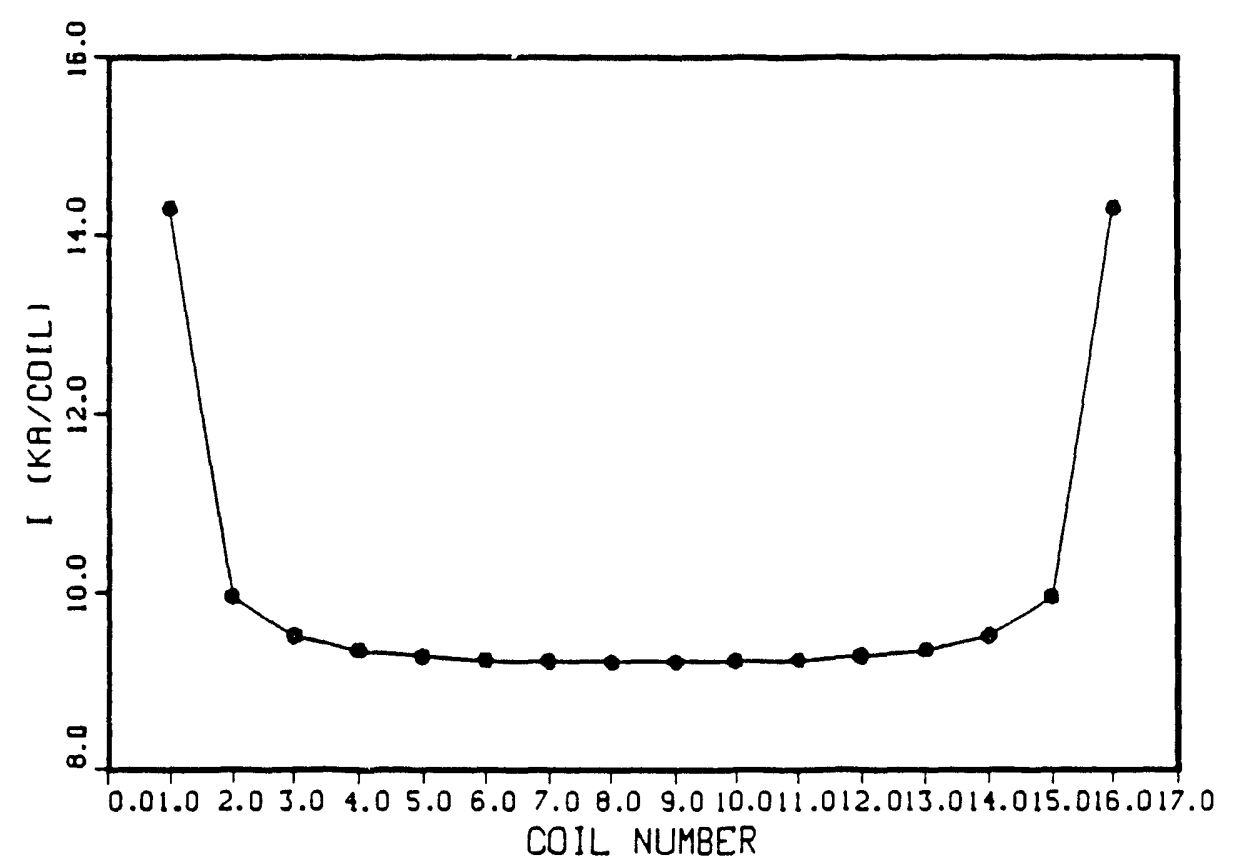

Figure 2.2 The current distribution of Maxwell coil system. 
TOROIDAL FIELD RIPPLE OF HBT

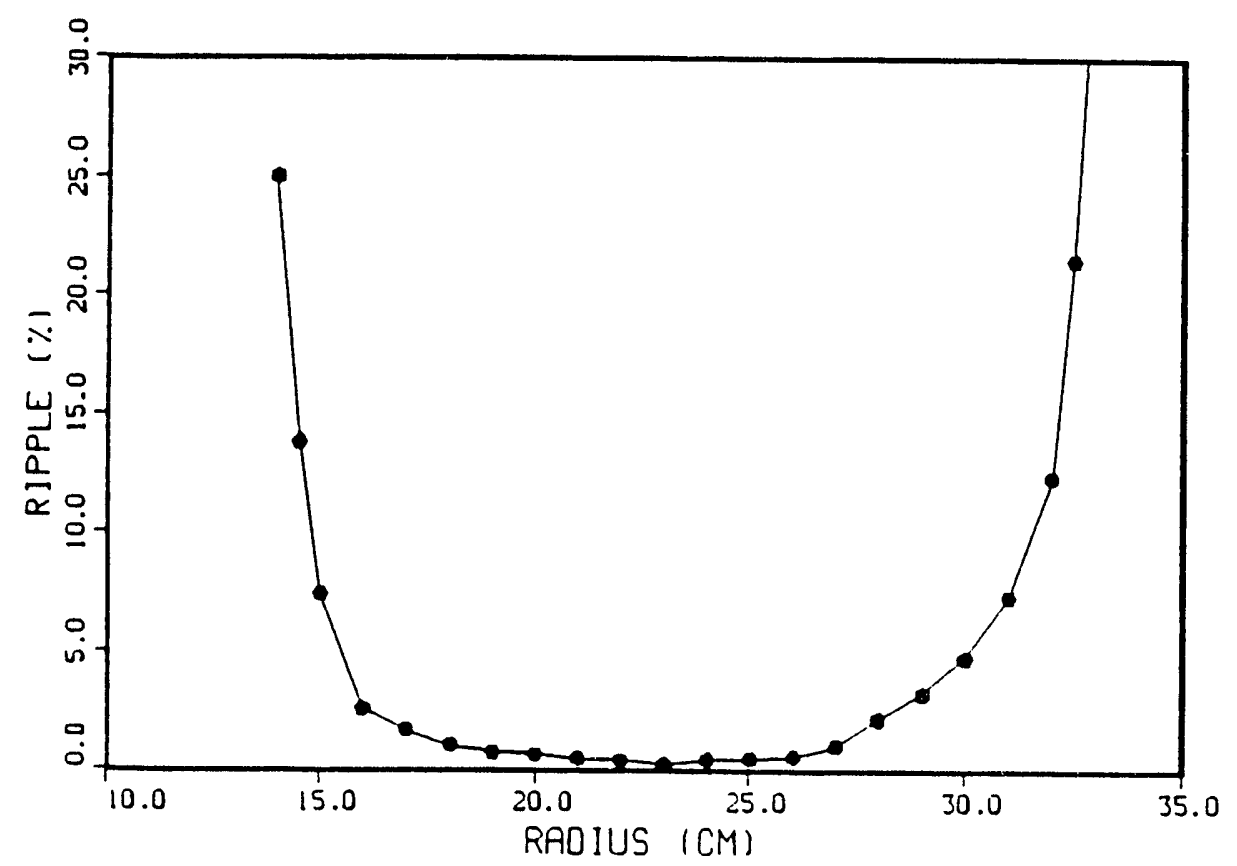

Figure 2.3 The ripple profile (peak to peak) of toroidal magnetic field at the location of diagnostic side-port. 


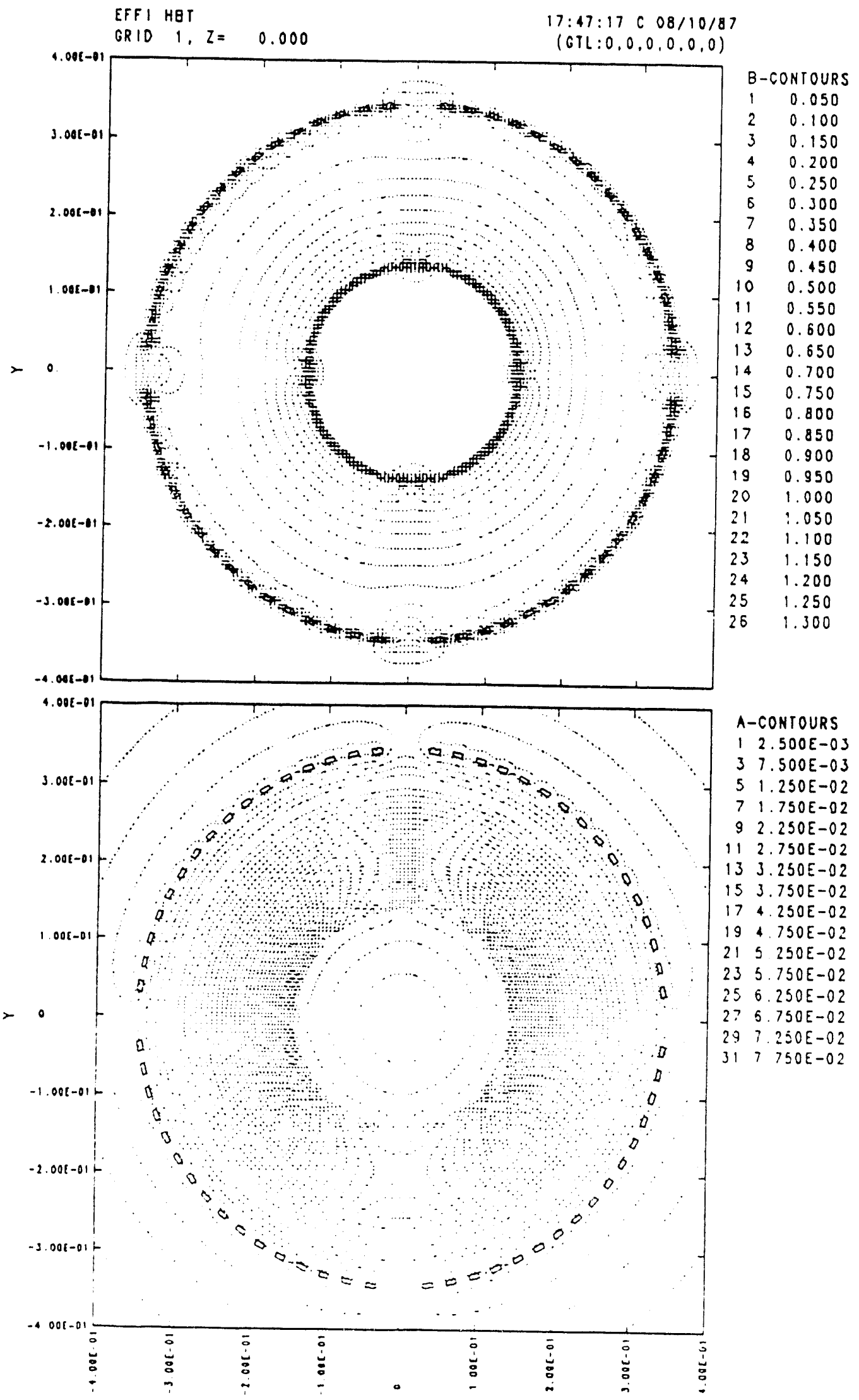

Figure 2.4 The $\mathrm{B}$ - and $\mathrm{A}$-contouirs of toróidal magnetic field resulting from the current distribution shown in Fig. 2.2. 


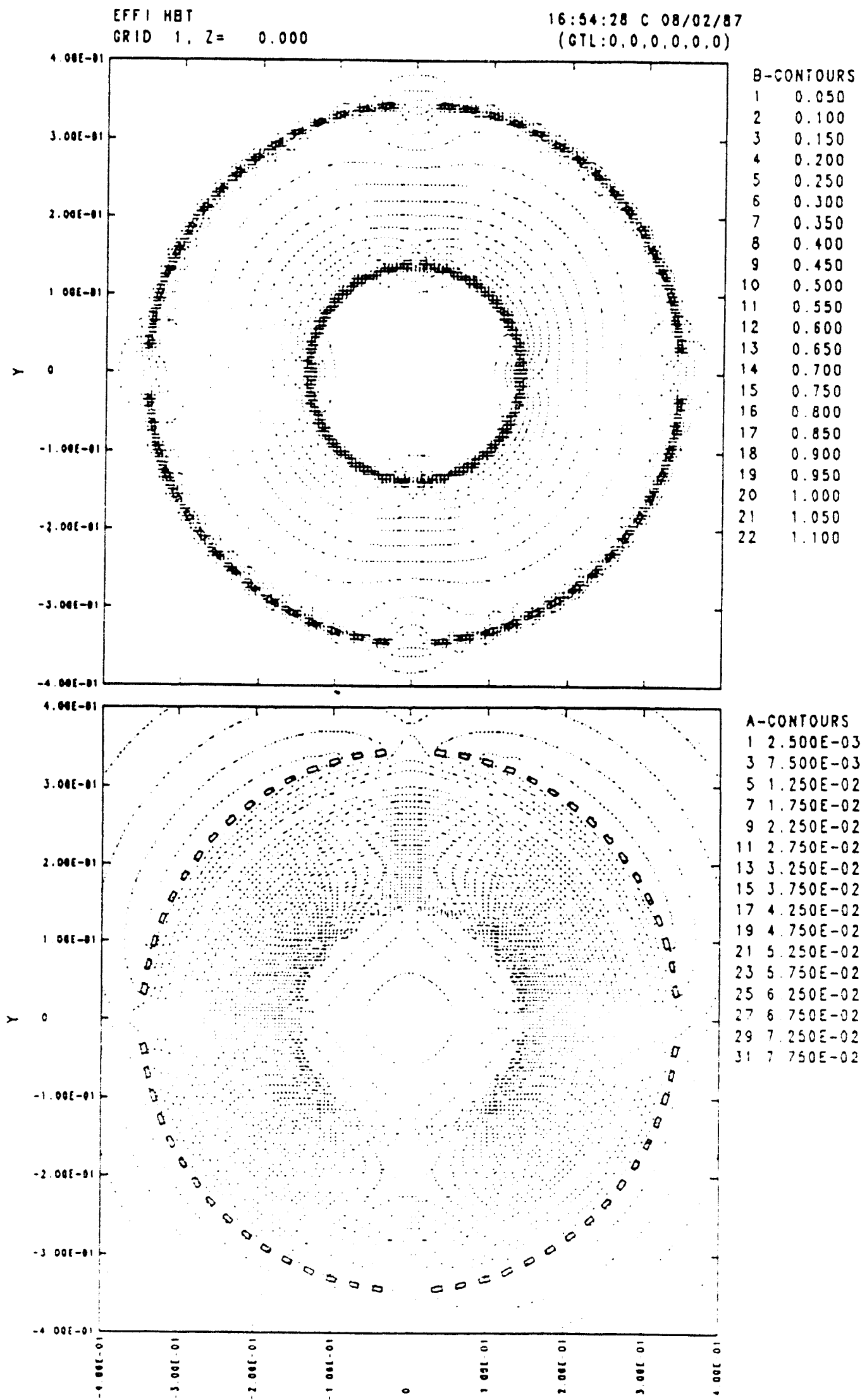

Figure 2.5 The B-and A-contours of toroidal magnetic field resulting from a uniform current distribution. 

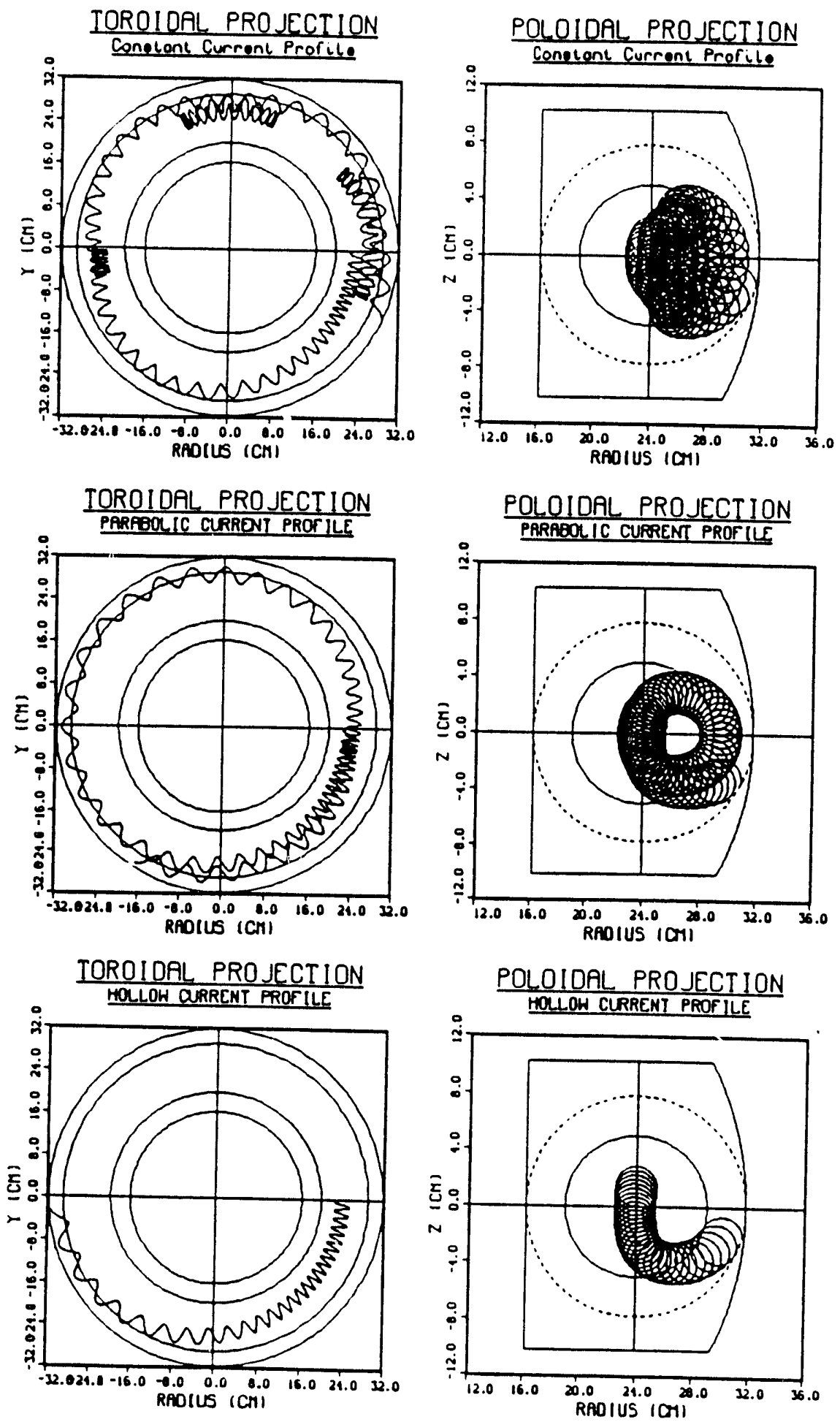

$$
\begin{array}{cccc}
B_{t}=0.3 T & I_{P}=10.0 \mathrm{kA} & r_{i}=0.0 & \theta_{i}=+10^{\circ} \\
\eta=0.0 & \delta_{0}=0.0 & \xi_{a}=0.0 & E=0.0
\end{array}
$$

Figure 2.6 The simulations under the condition of zero electric field. Compare with rig. 2.11 where the electric ficld is not equal to zero. 

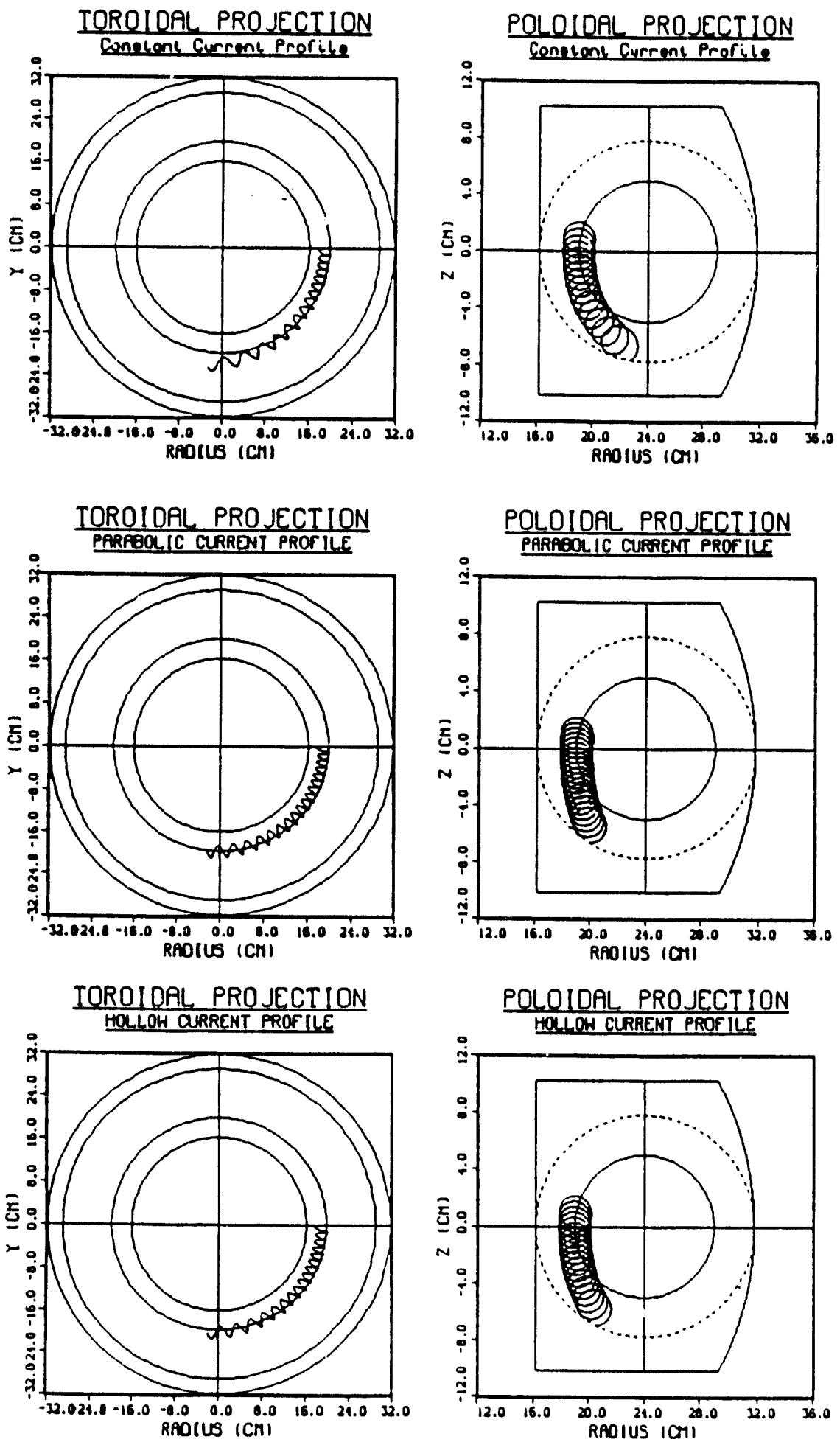

$$
\begin{array}{cccc}
B_{t}=0.3 T & I_{P}=10.0 \mathrm{kA} & r_{i}=-a & \theta_{i}=+10^{\circ} \\
\eta=0.0 & \delta_{0}=0.0 & \xi_{a}=0.0 & E \neq 0
\end{array}
$$

Figure 2.7 The simulation for the co-injection case where the initial ionization location is ri=-a. 

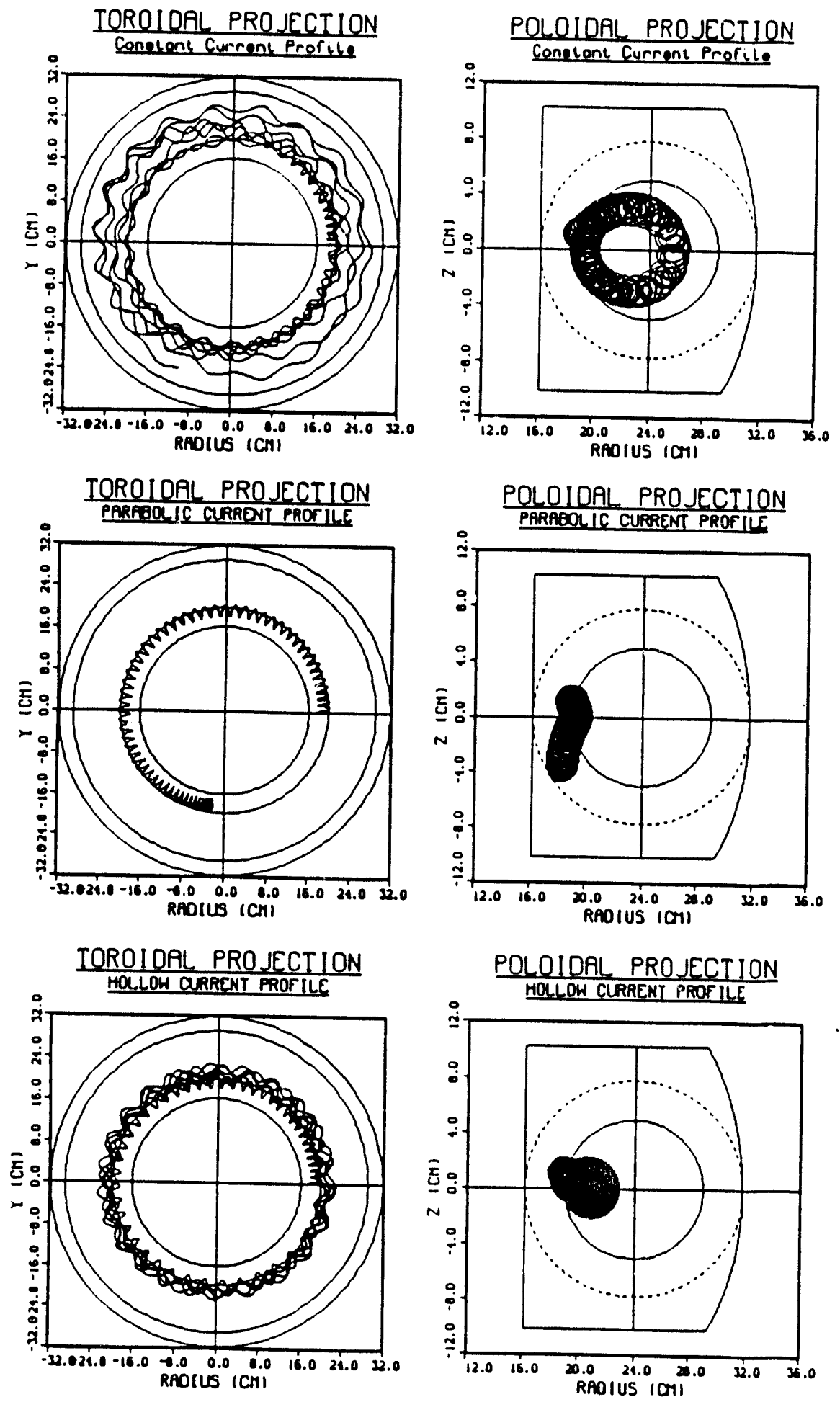

$$
\begin{array}{cccc}
B_{t}=0.3 T & I_{P}=10.0 \mathrm{kA} & r_{i}=-a & \theta_{i}=-10^{\circ} \\
\eta=0.0 & \delta_{0}=0.0 & \xi_{a}=0.0 & E \neq 0
\end{array}
$$

Figure 2.8 The simulation for the counter-injection case where the initial ionization location is $r=-a$. 

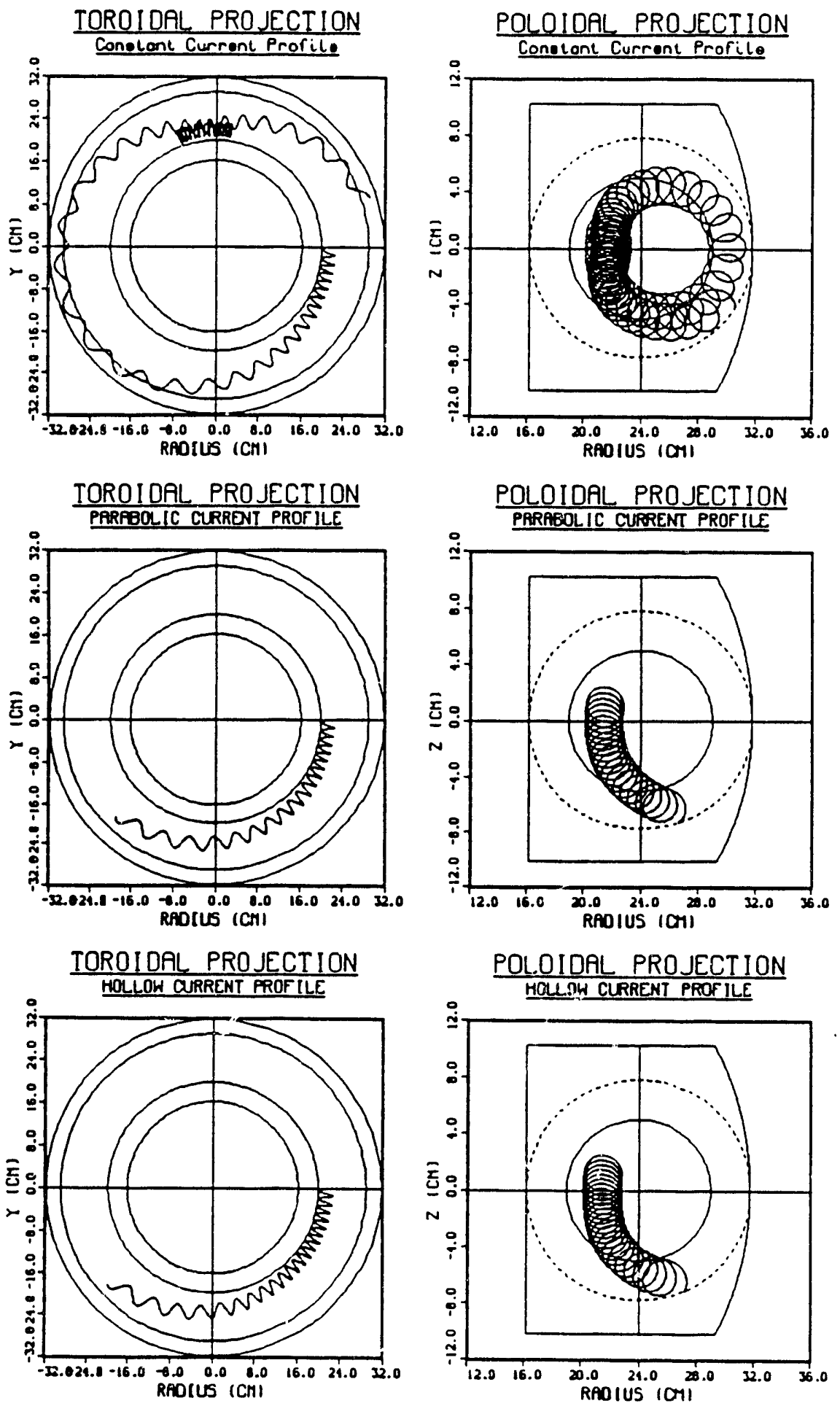

$$
\begin{array}{cccc}
B_{t}=0.3 T & I_{P}=10.0 \mathrm{kA} & r_{i}=-a / 2 & \theta_{i}=+10^{\circ} \\
\eta=0.0 & \delta_{0}=0.0 & \xi_{a}=0.0 & E \neq 0
\end{array}
$$

Figure 2.9 The simulation for the co-injection case where the initial ionization location $;$ s ri $=-a / 2$. 

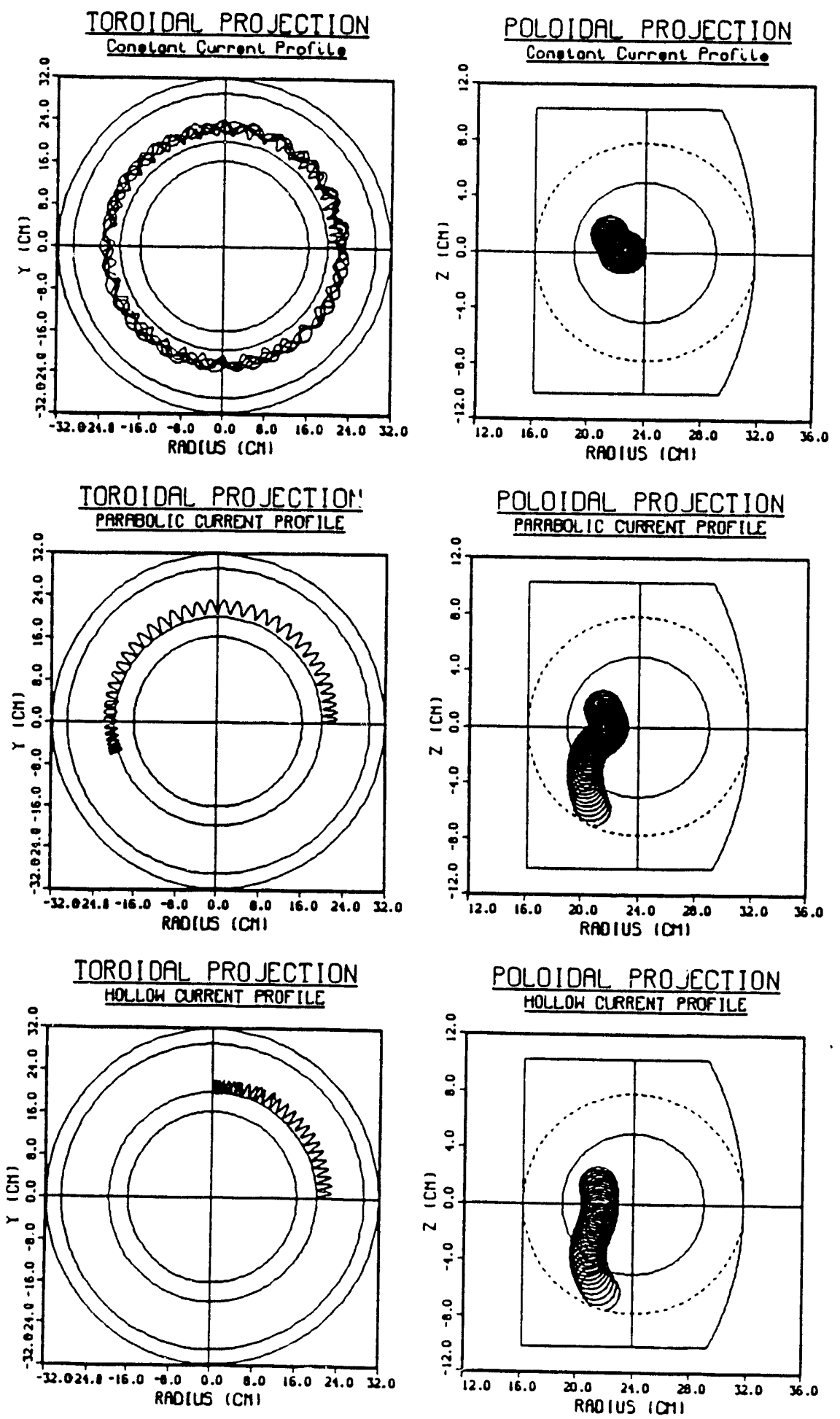

$$
\begin{array}{cccc}
B_{t}=0.3 T & I_{P}=10.0 \mathrm{kA} & r_{i}=-a / 2 & \theta_{i}=-10^{\circ} \\
\eta=0.0 & \delta_{0}=0.0 & \xi_{a}=0.0 & E \neq 0
\end{array}
$$

Figure 2.10 The simulation for the counter-injection case where the initial ionization location is $r=-a / 2$. 

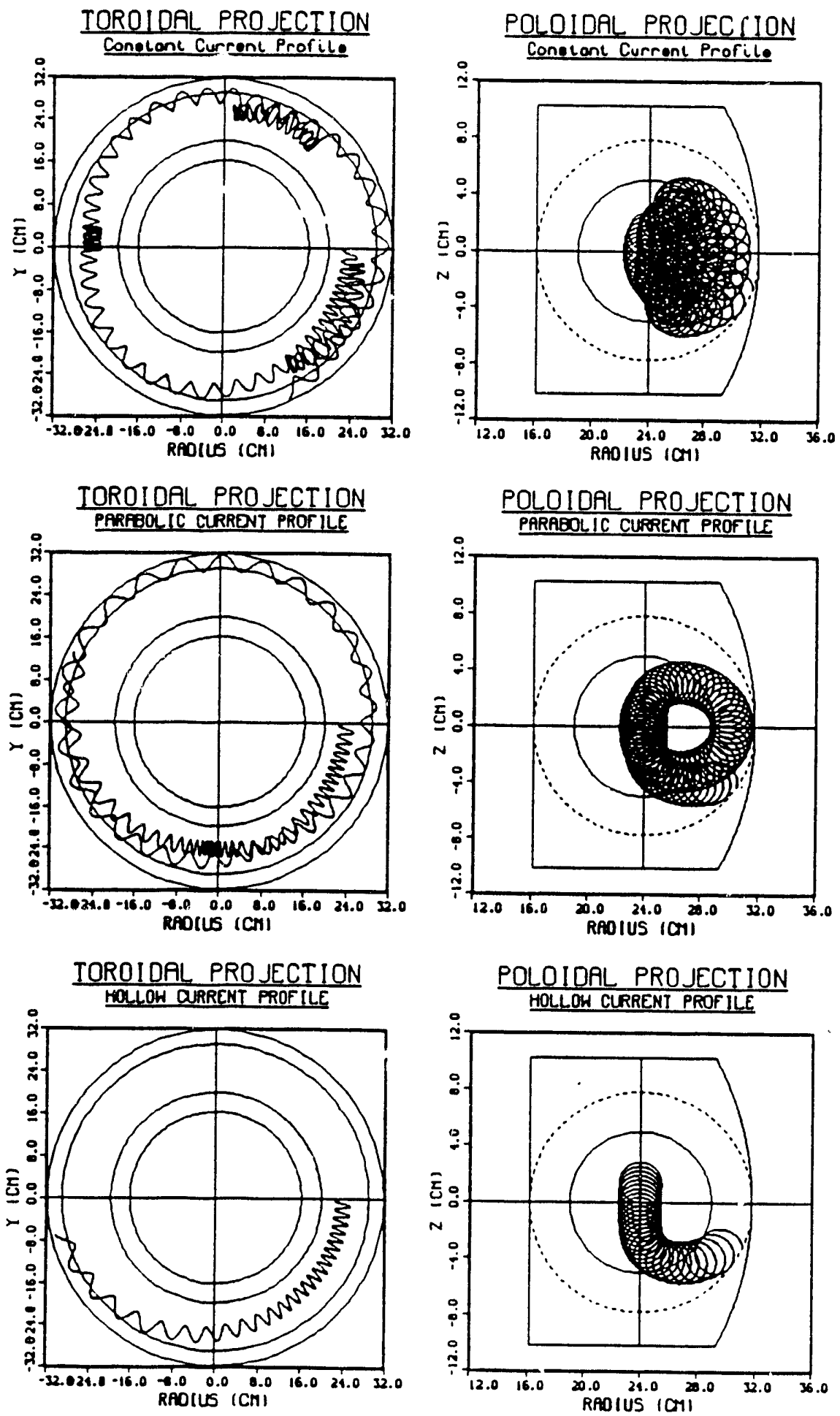

$$
\begin{array}{cccc}
B_{t}=0.3 T & I_{P}=10.0 k A & r_{i}=0.0 & \theta_{i}=+10^{\circ} \\
\eta=0.0 & \delta_{0}=0.0 & \xi_{a}=0.0 & E \neq 0
\end{array}
$$

Figure 2.11 The simulation for the co-injection case where the initial ionization location is $r=0$. 

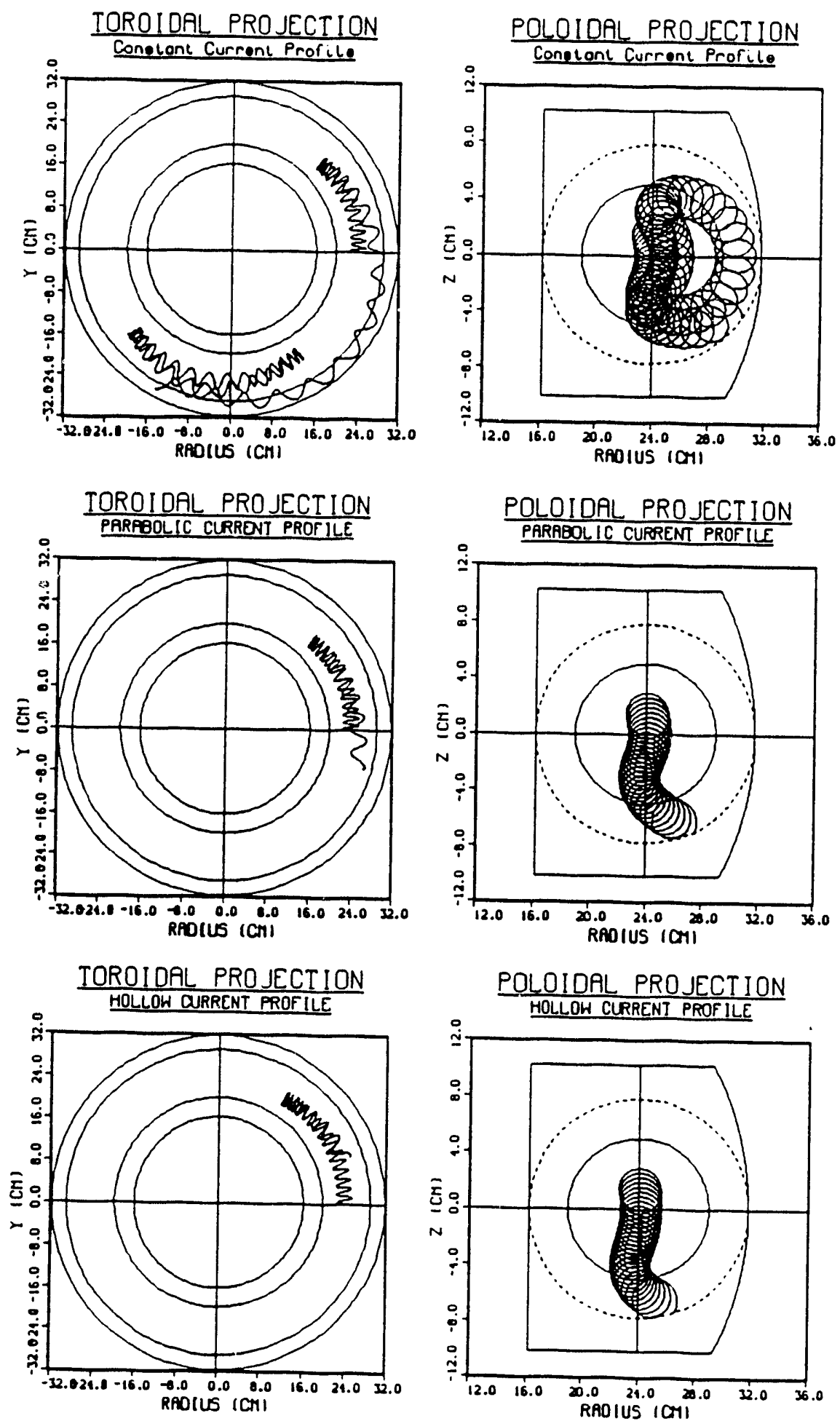

$$
\begin{array}{cccc}
B_{t}=0.3 T & I_{P}=10.0 \mathrm{kA} & r_{i}=0.0 & \theta_{i}=-10^{\circ} \\
\eta=0.0 & \delta_{0}=0.0 & \xi_{a}=0.0 & E \neq 0
\end{array}
$$

Figure 2.12 The simulation for the counter-injection case where the initial ionization location is ri $=0$. 

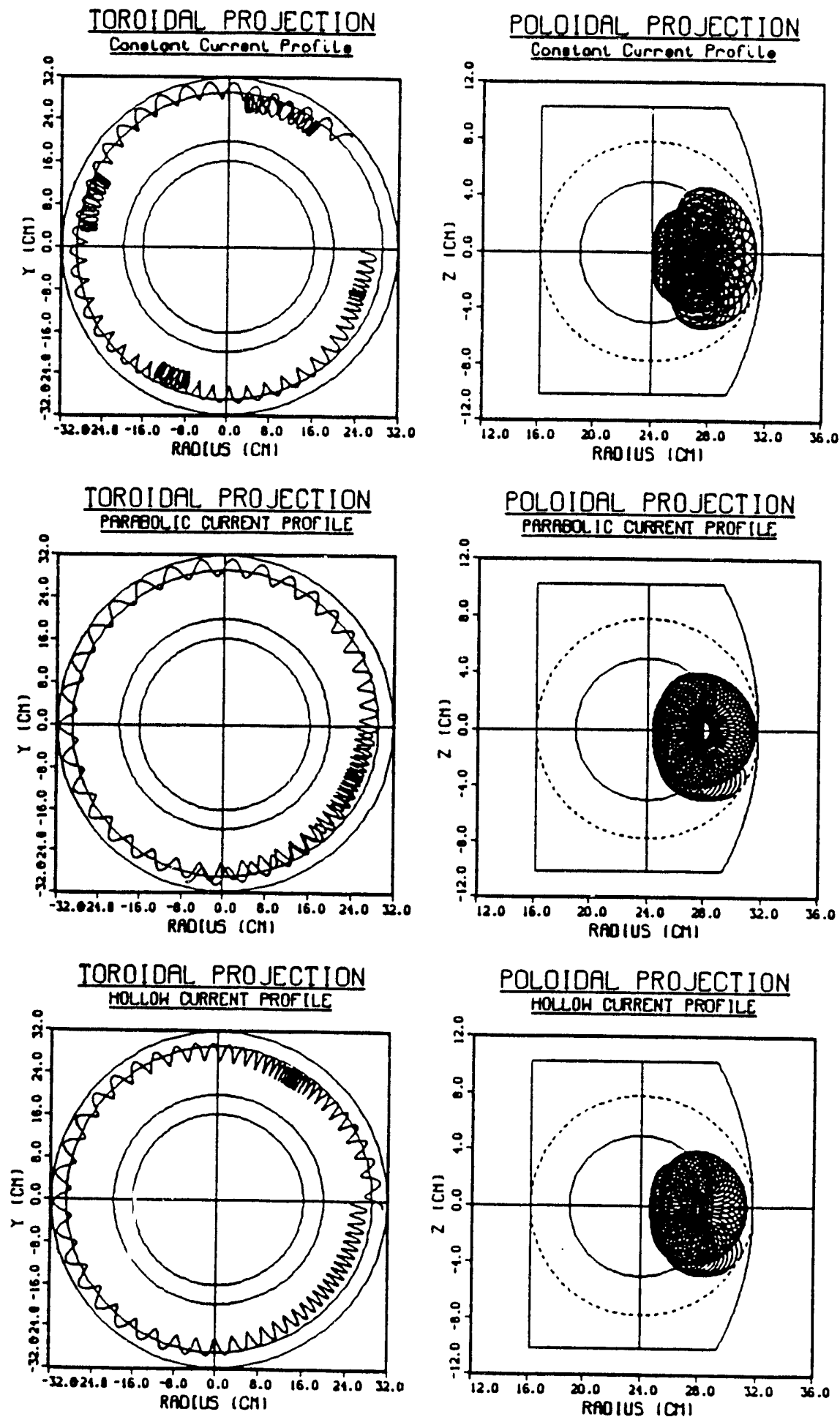

$$
\begin{array}{cccc}
B_{t}=0.3 T & I_{P}=10.0 \mathrm{kA} & r_{i}=+a / 2 & \theta_{i}=+10^{\circ} \\
\eta=0.0 & \delta_{0}=0.0 & \xi_{a}=0.0 & E \neq 0
\end{array}
$$

Figure 2.13 The simulation for the co-injection case where the initial ionization location is $r i=+a / 2$. 

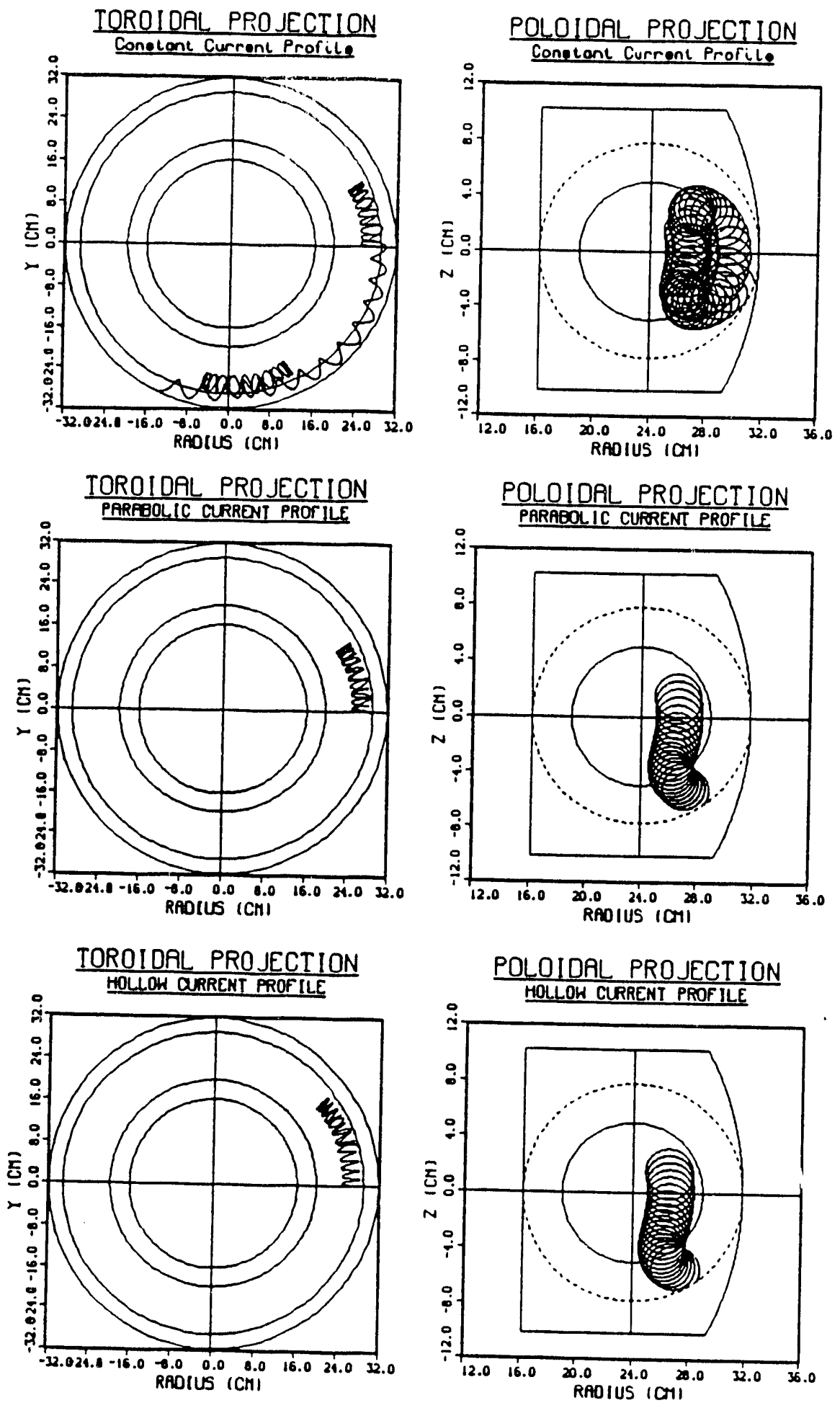

$$
\begin{array}{cccc}
B_{t}=0.3 T & I_{P}=10.0 \mathrm{kA} & r_{i}=+a / 2 & \theta_{i}=-10^{\circ} \\
\eta=0.0 & \delta_{0}=0.0 & \xi_{a}=0.0 & E \neq 0
\end{array}
$$

Figure 2.14 The simulation for the counter-injection case where the initial ionization location is $r i=+a / 2$. 

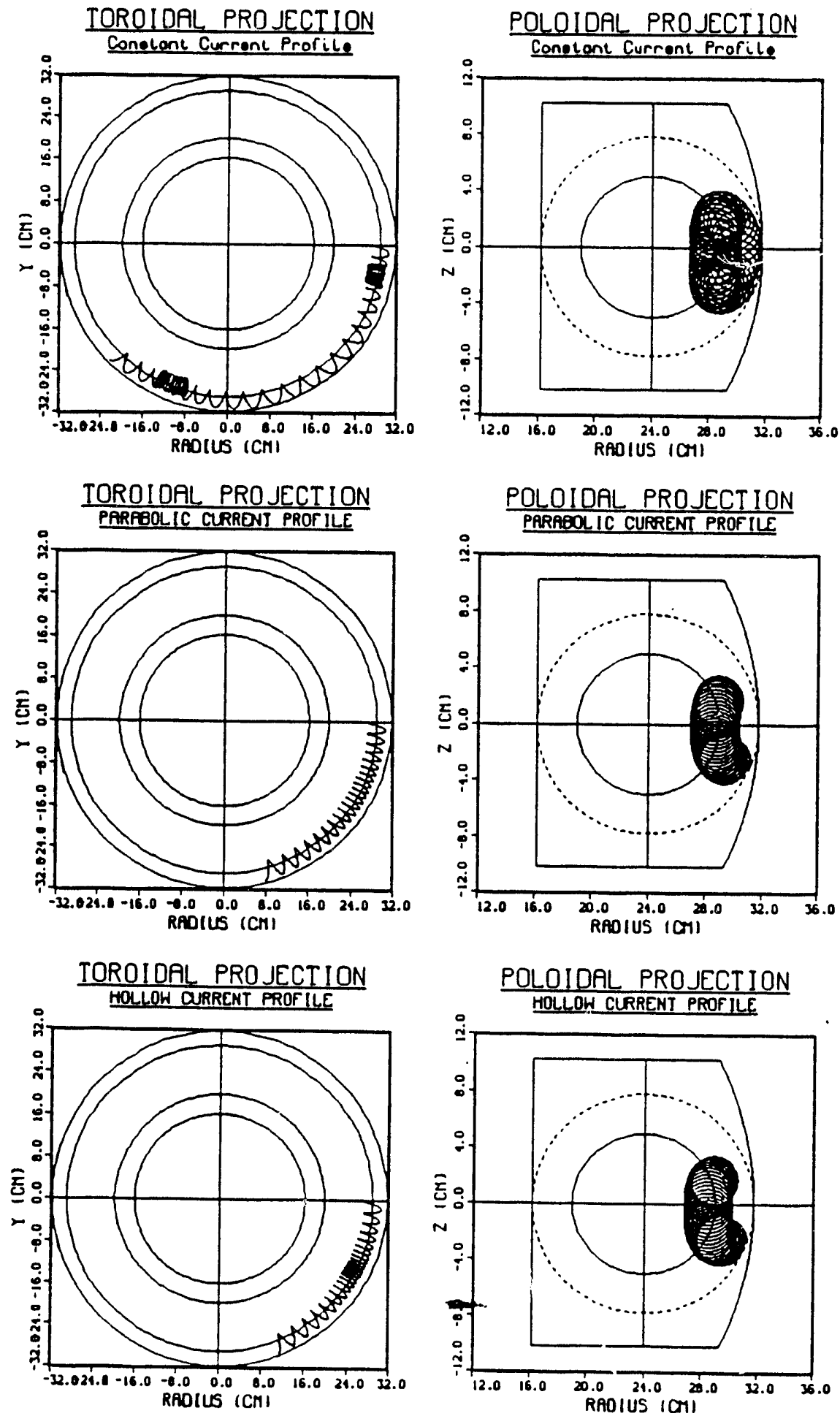

$$
\begin{array}{cccc}
B_{t}=0.3 T & I_{P}=10.0 \mathrm{kA} & r_{i}=+a & \theta_{i}=+10^{\circ} \\
\eta=0.0 & \delta_{0}=0.0 & \xi_{a}=0.0 & E \neq 0
\end{array}
$$

Figure 2.15 The simulation for the co-injection case where the initial ionization location is $\mathrm{r}=\mathrm{a}$. 

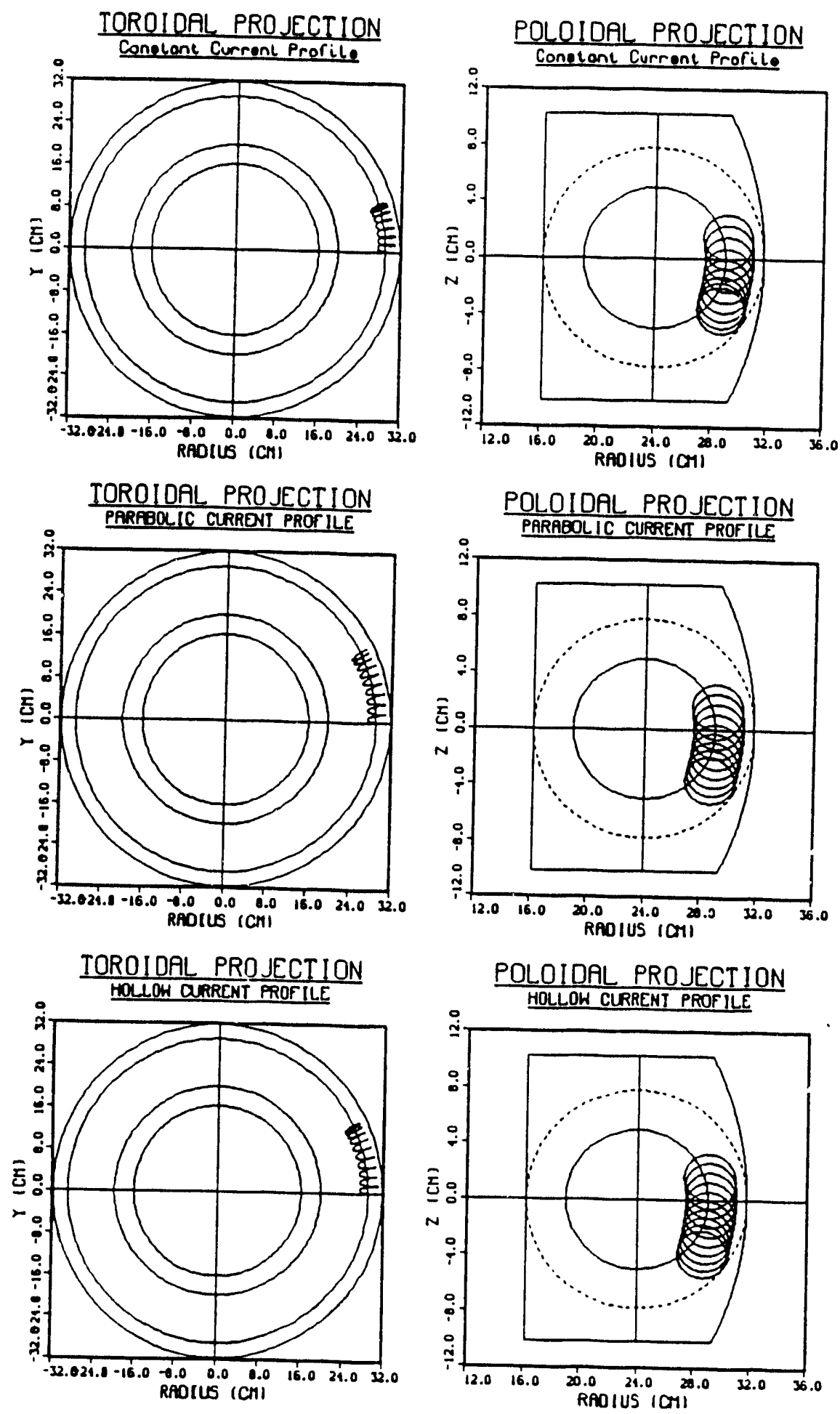

$$
\begin{array}{cccc}
B_{t}=0.3 T & I_{P}=10.0 \mathrm{kA} & r_{i}=+a & \theta_{i}=-10^{\circ} \\
\eta=0.0 & \delta_{0}=0.0 & \xi_{a}=0.0 & E \neq 0
\end{array}
$$

Figure 2.16 The simulation for the counter-injection case where the initial ionization location is $r i=a$. 

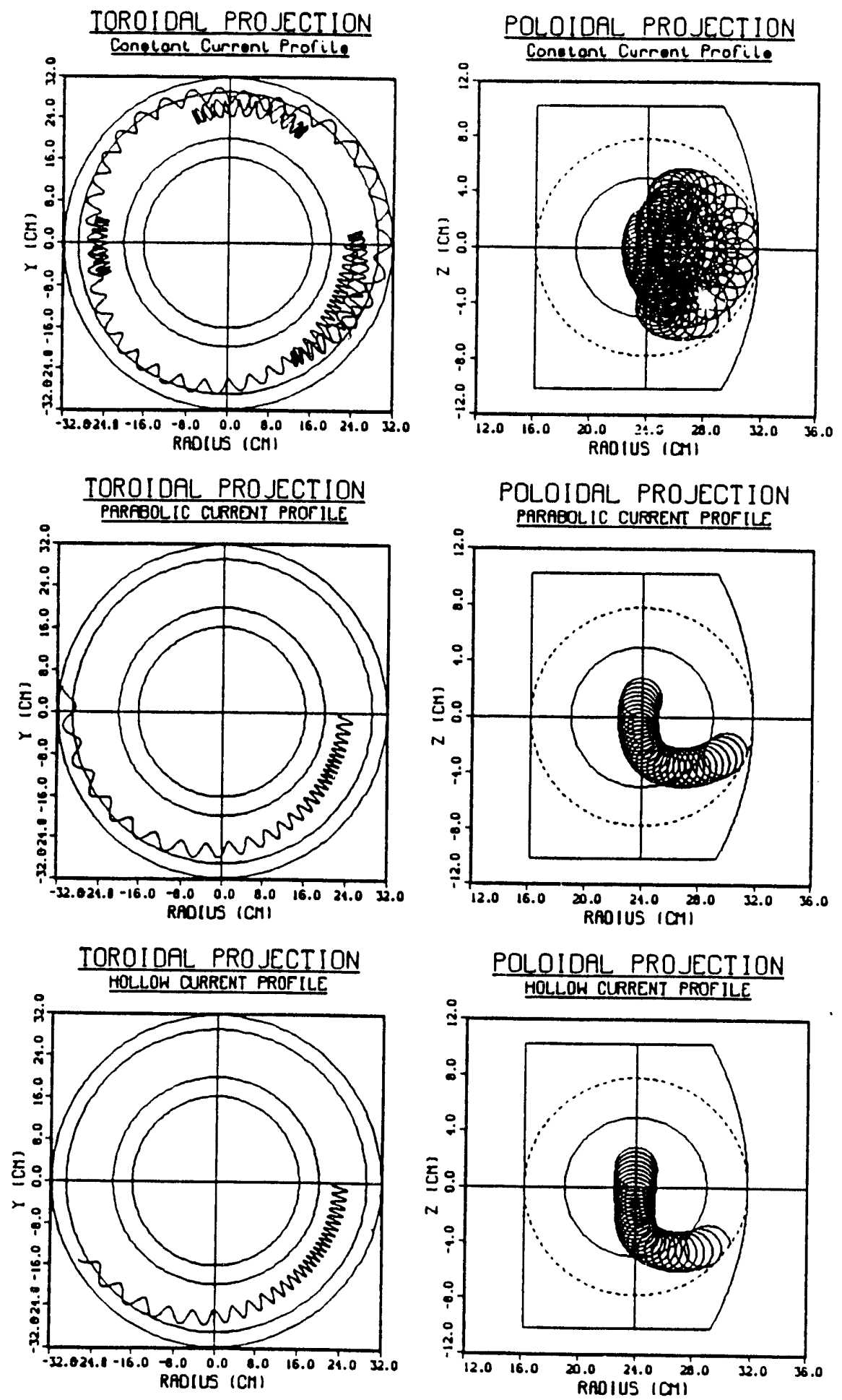

$$
\begin{array}{cccc}
B_{t}=0.3 T & I_{P}=10.0 \mathrm{kA} & r_{i}=0.0 & \theta_{i}=+10^{\circ} \\
\eta=10 \% & \delta_{0}=1 \% & \xi_{a}=0.0 & E \neq 0
\end{array}
$$

Figure 2.17 The simulation for the co-injection case where the ringing level is $10 \%$ and ripple level is $1 \%$. Compare with

Fig. 2.11 and Fig. 2.19. 

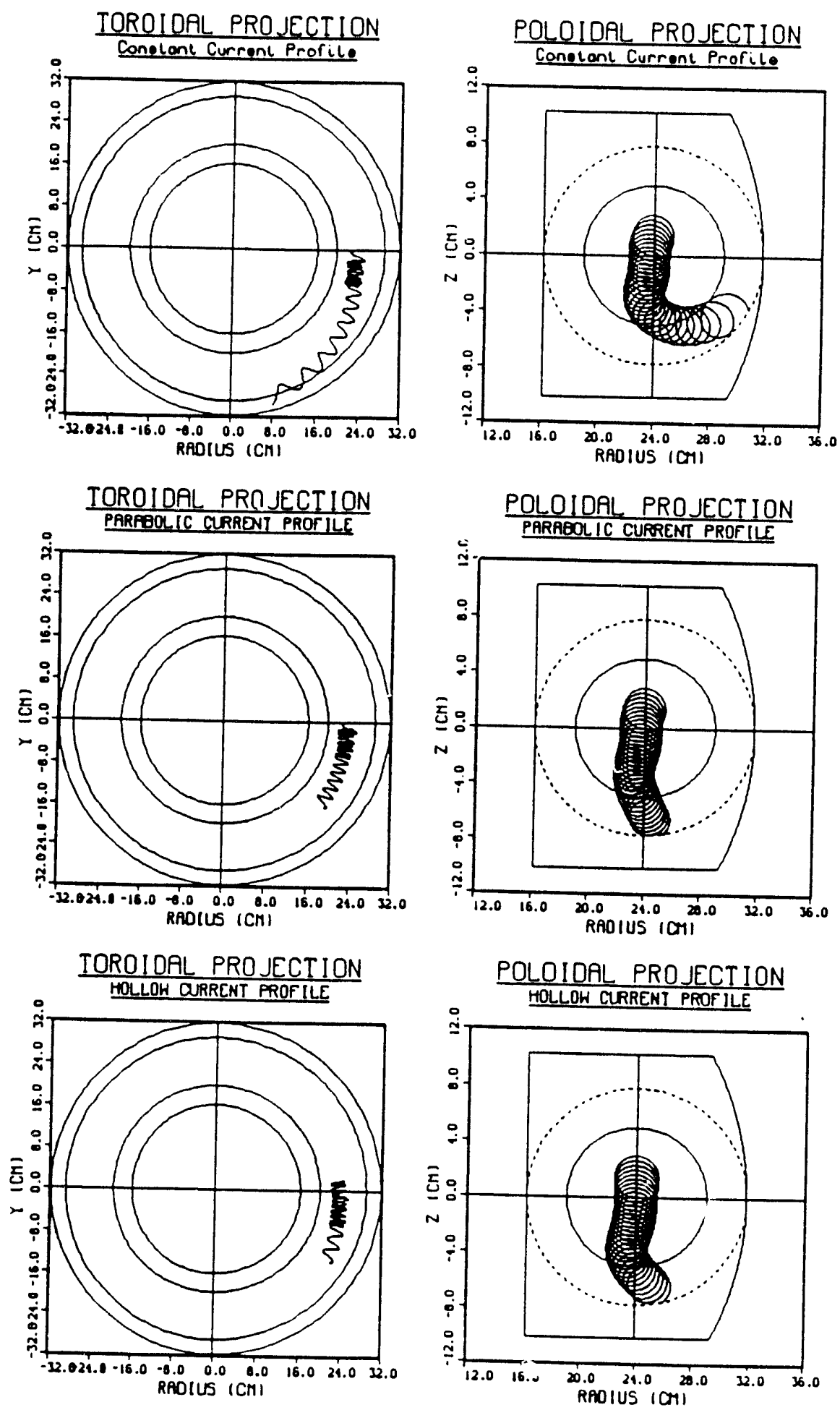

$$
\begin{array}{cccc}
B_{t}=0.3 T & I_{P}=16.0 \mathrm{kA} & r_{i}=0.0 & \theta_{i}=+10^{\circ} \\
\eta=10 \% & \delta_{0}=5 \% & \xi_{a}=0.0 & E \neq 0
\end{array}
$$

Figure 2,18 The simulation for the co-injection case where the ringing level is $10 \%$ and ripple level is $5 \%$. Compare with Fig. 2.11 and Fig. 2.19. 

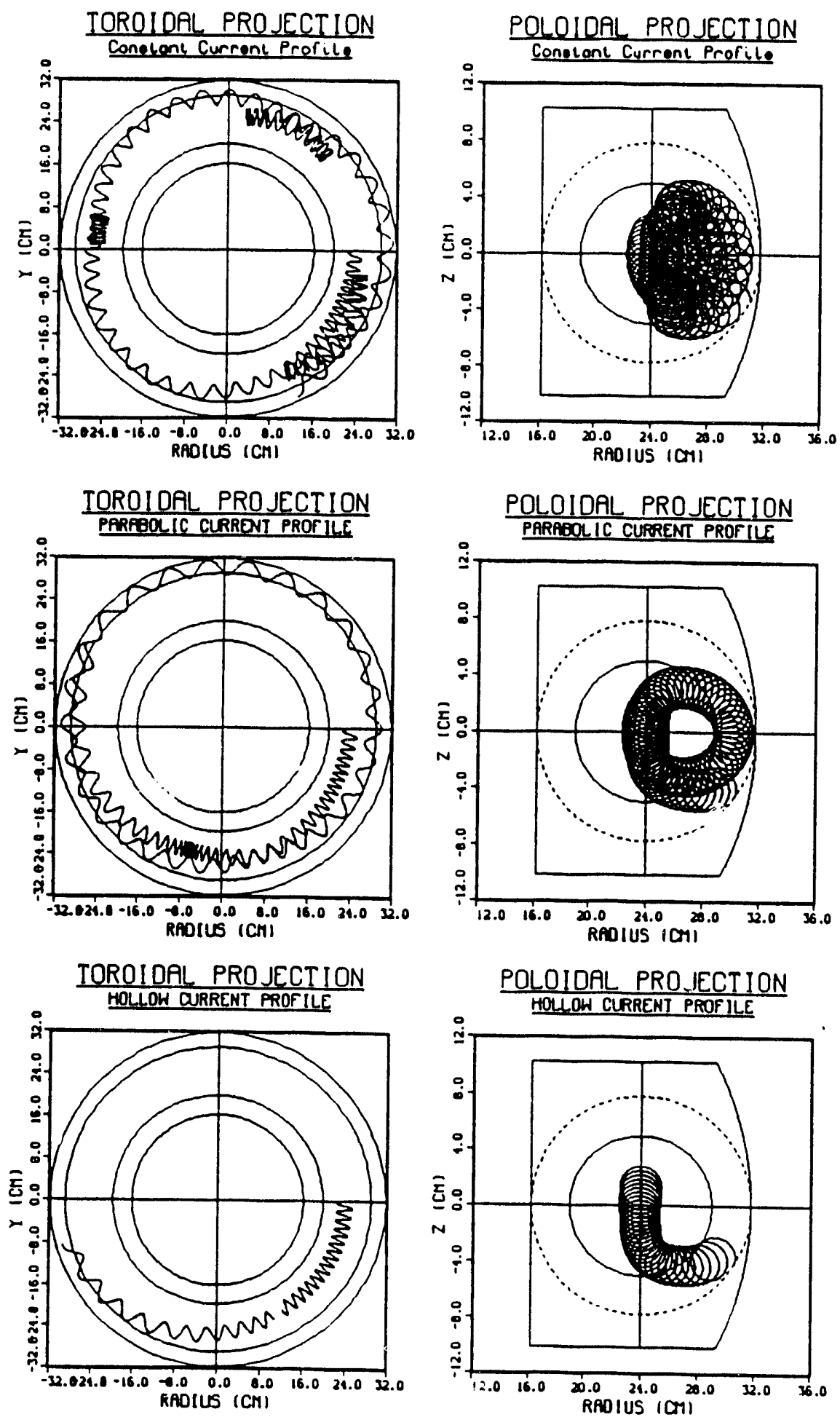

$$
\begin{array}{cccc}
B_{t}=0.3 T & I_{P}=10.0 \mathrm{kA} & r_{i}=0.0 & \theta_{i}=+10^{\circ} \\
\eta=10 \% & \delta_{0}=0.0 & \xi_{a}=0.0 & E \neq 0
\end{array}
$$

Figure 2.19 The simulation for the co-injection case where the ringing level is $10 \%$. Compare with Fig. 2.11. 

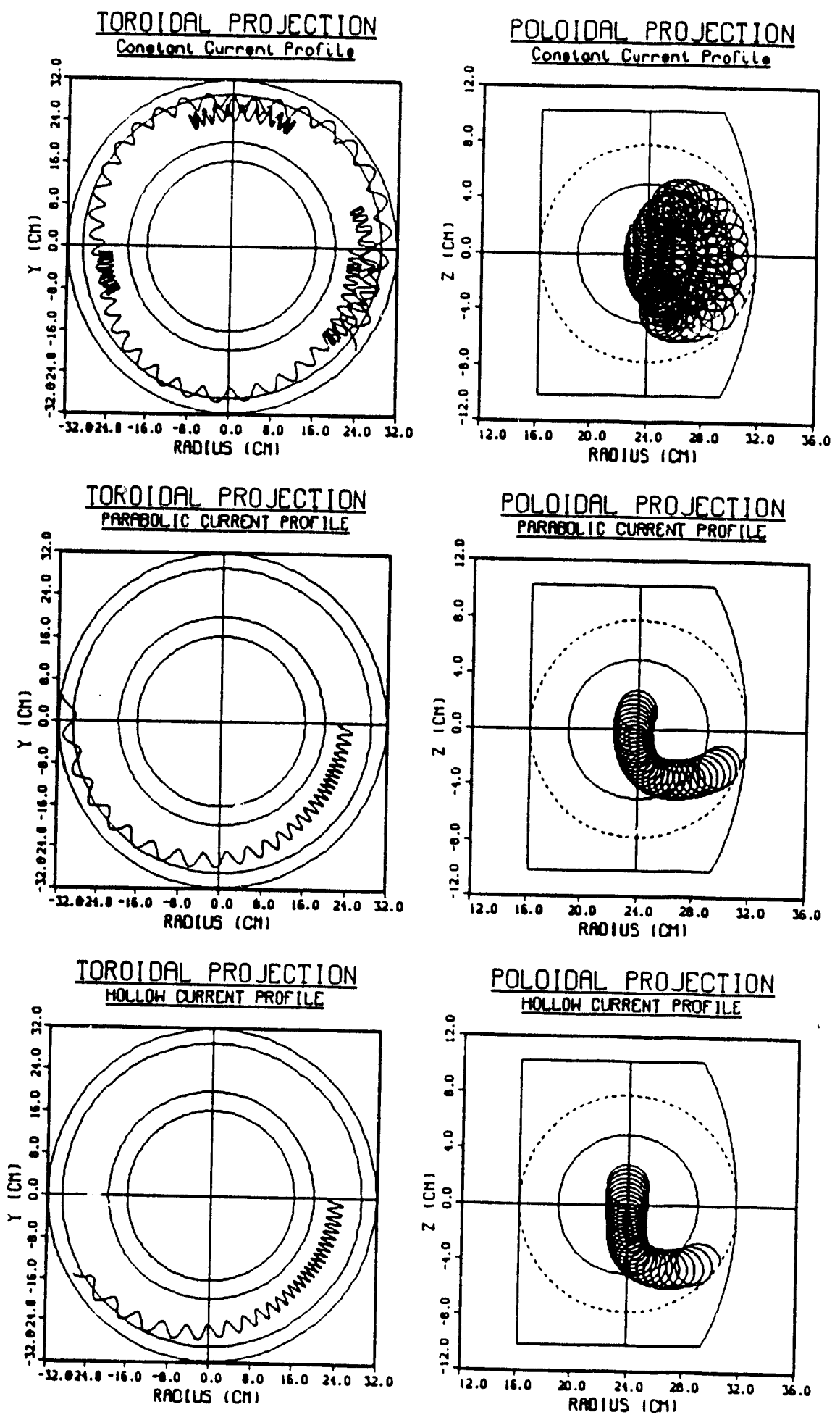

$$
\begin{array}{cccc}
B_{t}=0.3 T & I_{P}=10.0 \mathrm{kA} & r_{i}=0.0 & \theta_{i}=+10^{\circ} \\
\eta=10 \% & \delta_{0}=1 \% & \xi_{a}=0.1 \mathrm{~cm} & E \neq 0
\end{array}
$$

Fig uru 2.20 The simulation for the co-injection case where the kink displacement is $0.1 \mathrm{~cm}$. Compare with Fig. 2.17. 

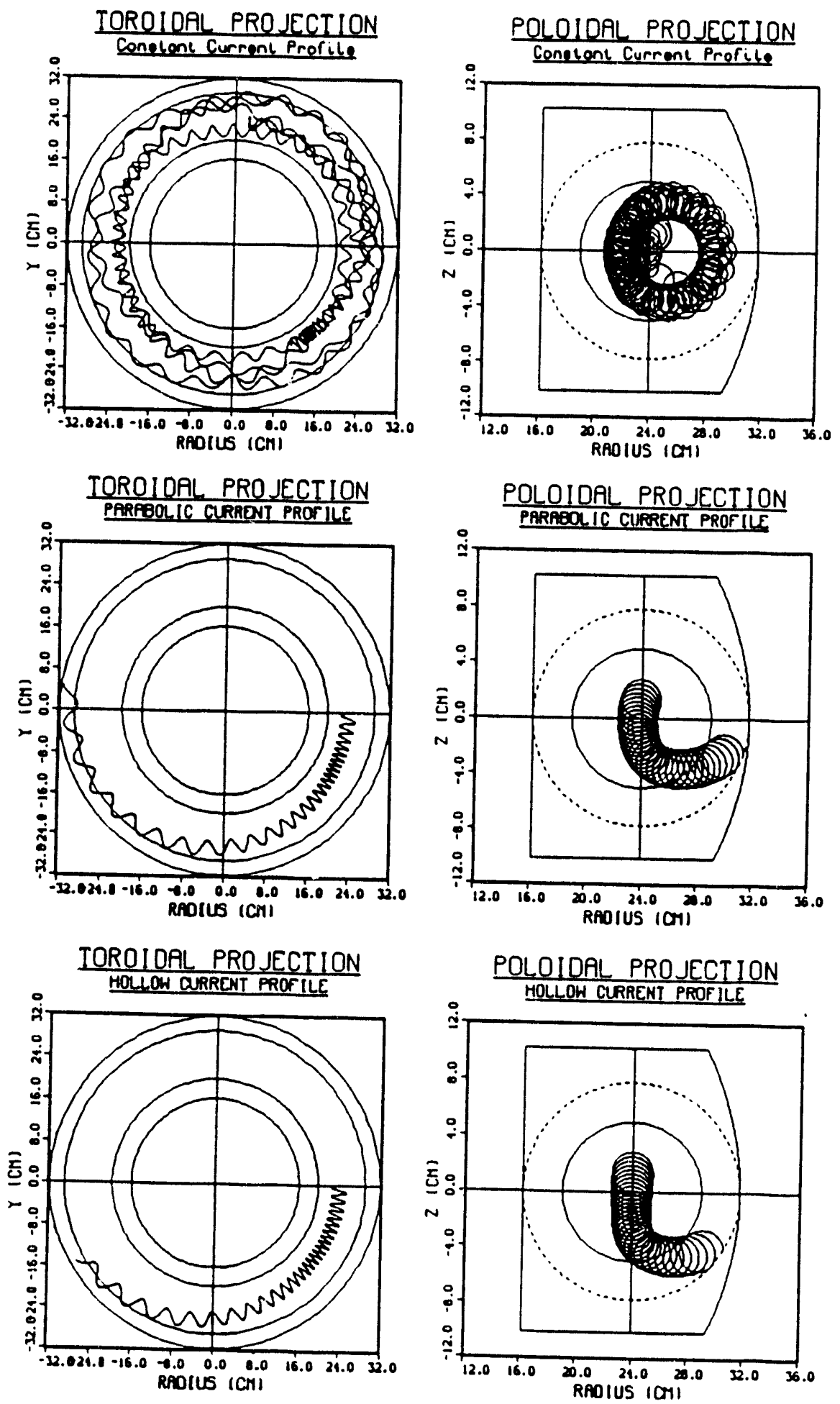

$$
\begin{array}{cccc}
B_{t}=0.3 T & I_{P}=10.0 \mathrm{kA} & r_{i}=0.0 & \theta_{i}=+10^{\circ} \\
\eta=10 \% & \delta_{0}=1 \% & \xi_{i}=1.0 \mathrm{~cm} & E \neq 0
\end{array}
$$

Figure 2.21 The simulatioñ for tor the co-injection case where the kink displacemeni is $1.0 \mathrm{~cm}$. Compare with Fig. 2.17. 

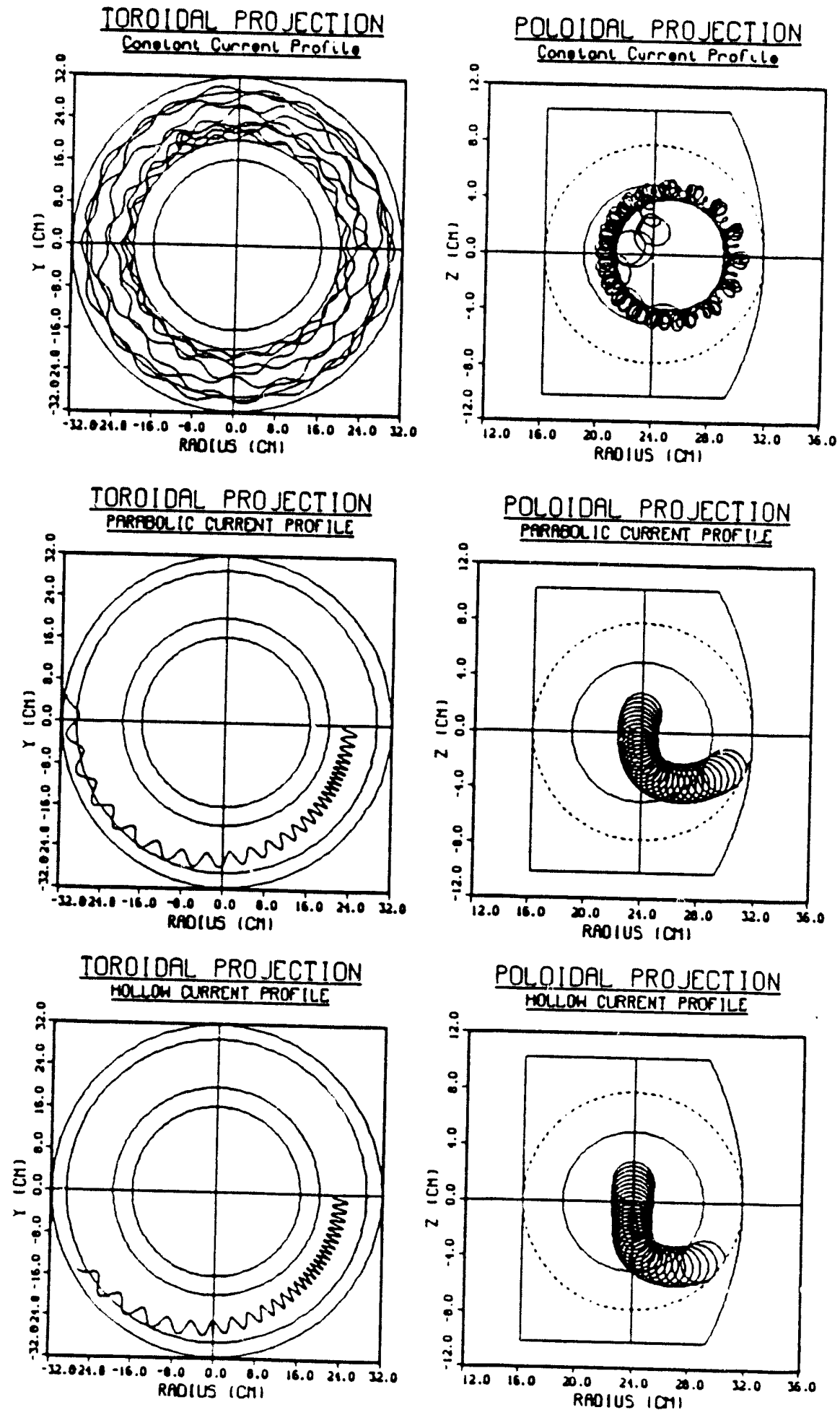

$$
\begin{array}{cccc}
B_{t}=0.3 T & I_{P}=10.0 \mathrm{kA} & r_{i}=0.0 & \theta_{i}=+10^{\circ} \\
\eta=10 \% & \delta_{0}=1 \% & \xi_{a}=2.0 \mathrm{~cm} & E \neq 0
\end{array}
$$

Figure 2.22 The simulation for the co-injection case where the kink displacement is $2.0 \mathrm{~cm}$. Compare with Fig. 2.17 . 

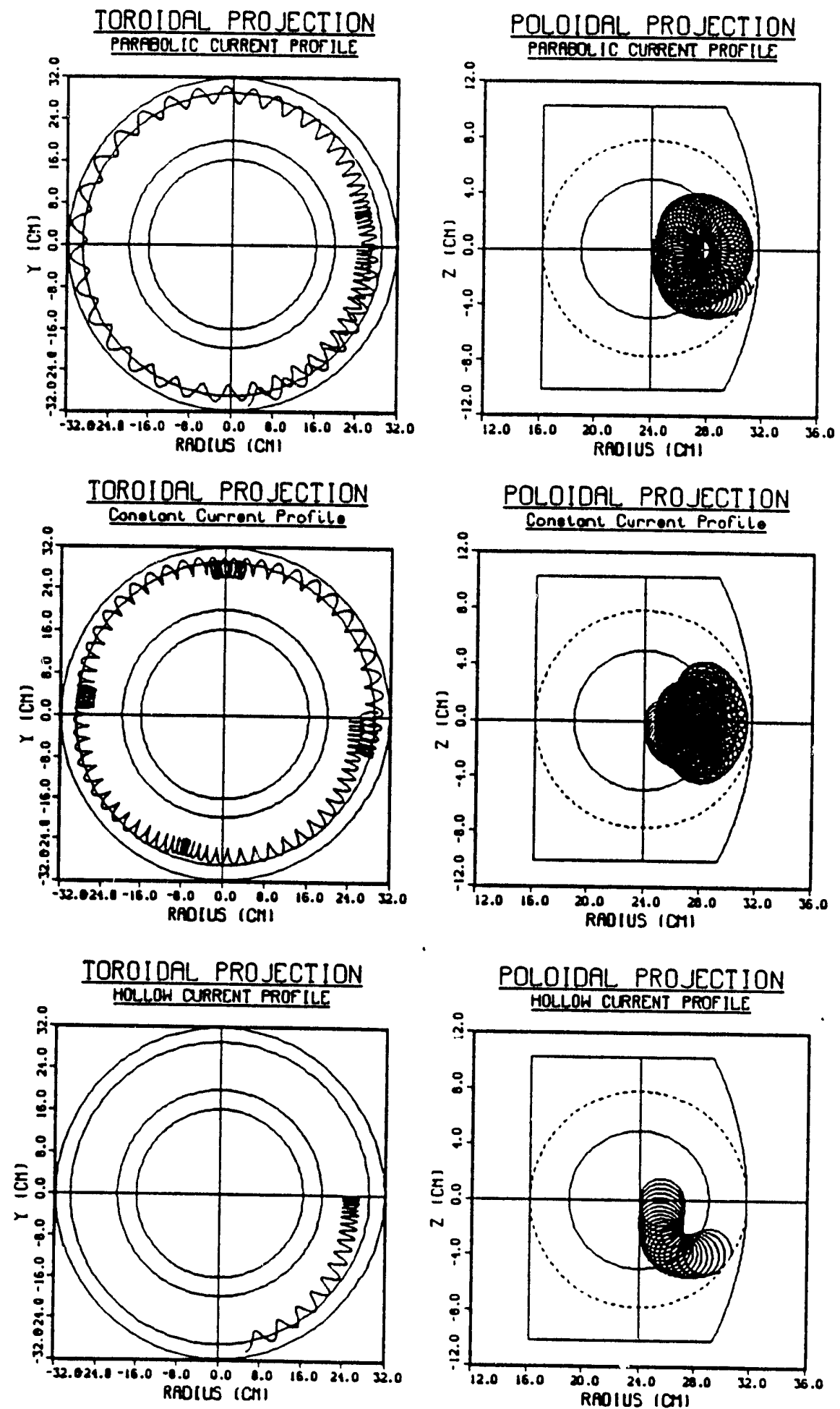

$$
\begin{array}{cccc}
B_{t}=0.3 T & I_{P}=10.0 k A & r_{i}=0.0 & \theta_{i}=0.0 \\
\eta=0.0 & \delta_{0}=0.0 & \xi_{a}=0.0 & E \neq 0
\end{array}
$$

Figure 2.23 The simulation for the vertical injection case under the ideal condition. Compare with Fig. 2.11. 

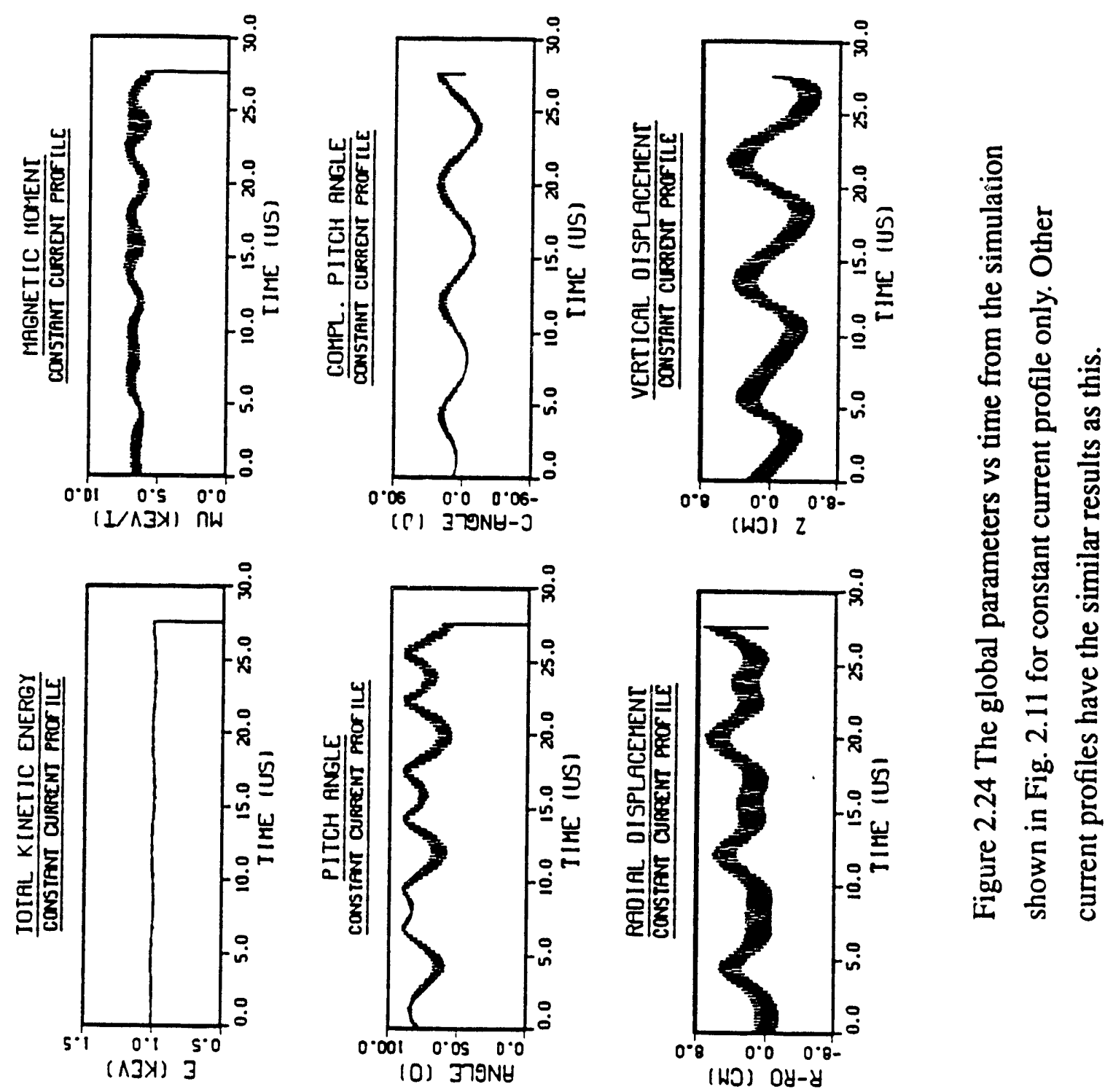


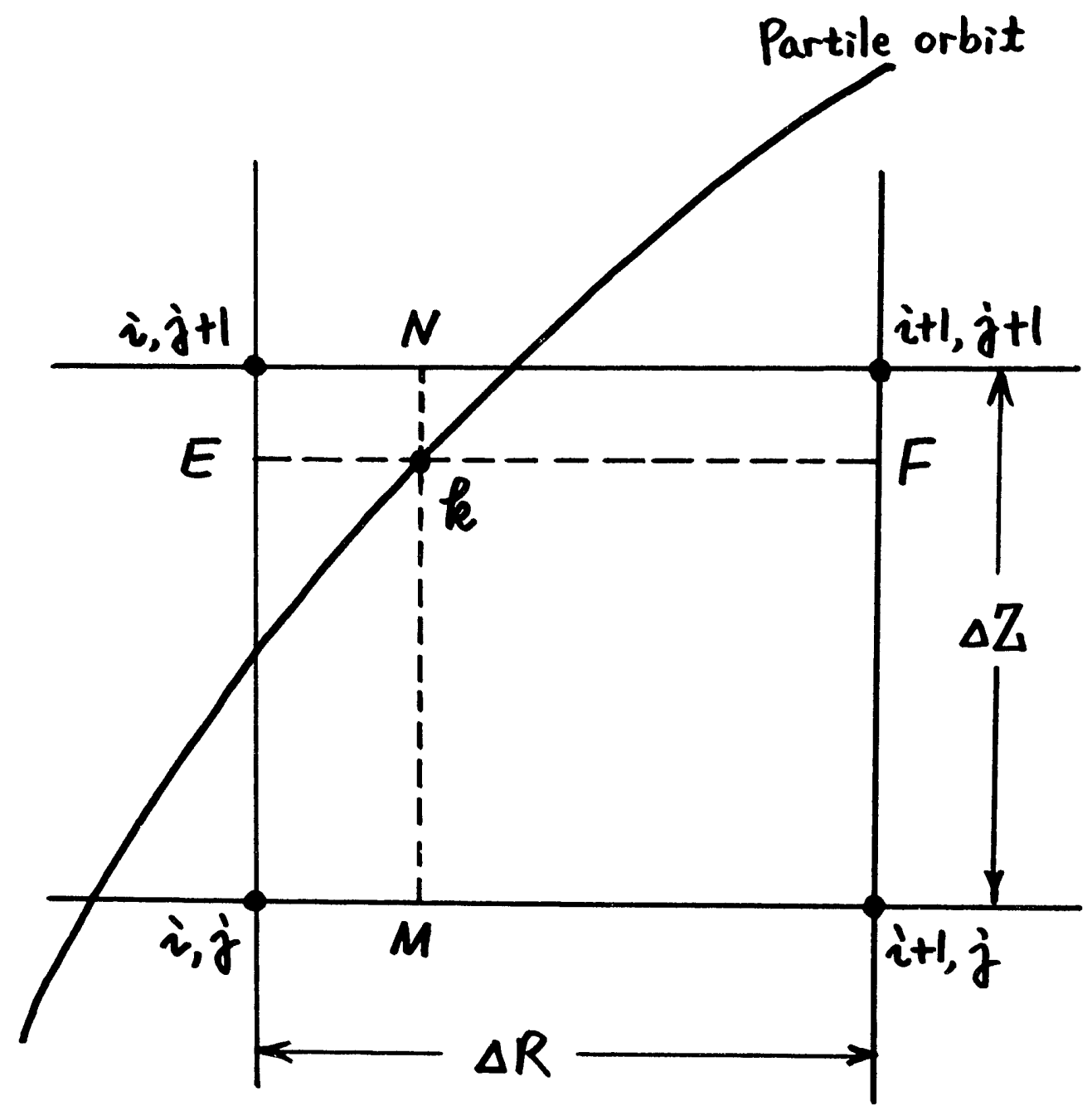

Figure 2.25 The Taylor interpolation relation. 

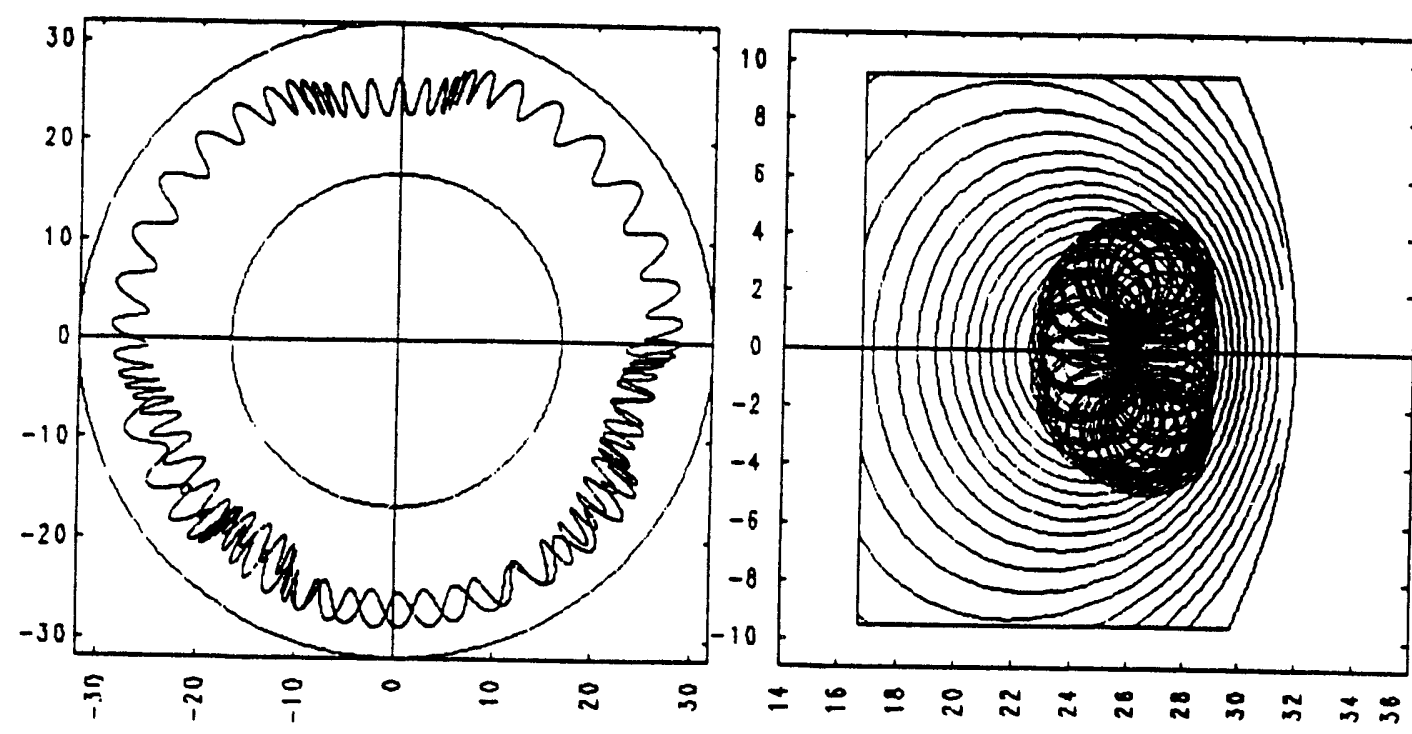

$R_{0}=25.0(\mathrm{~cm})$

$\theta_{i}=+14^{\circ}$

$T=25.0(\mu s)$
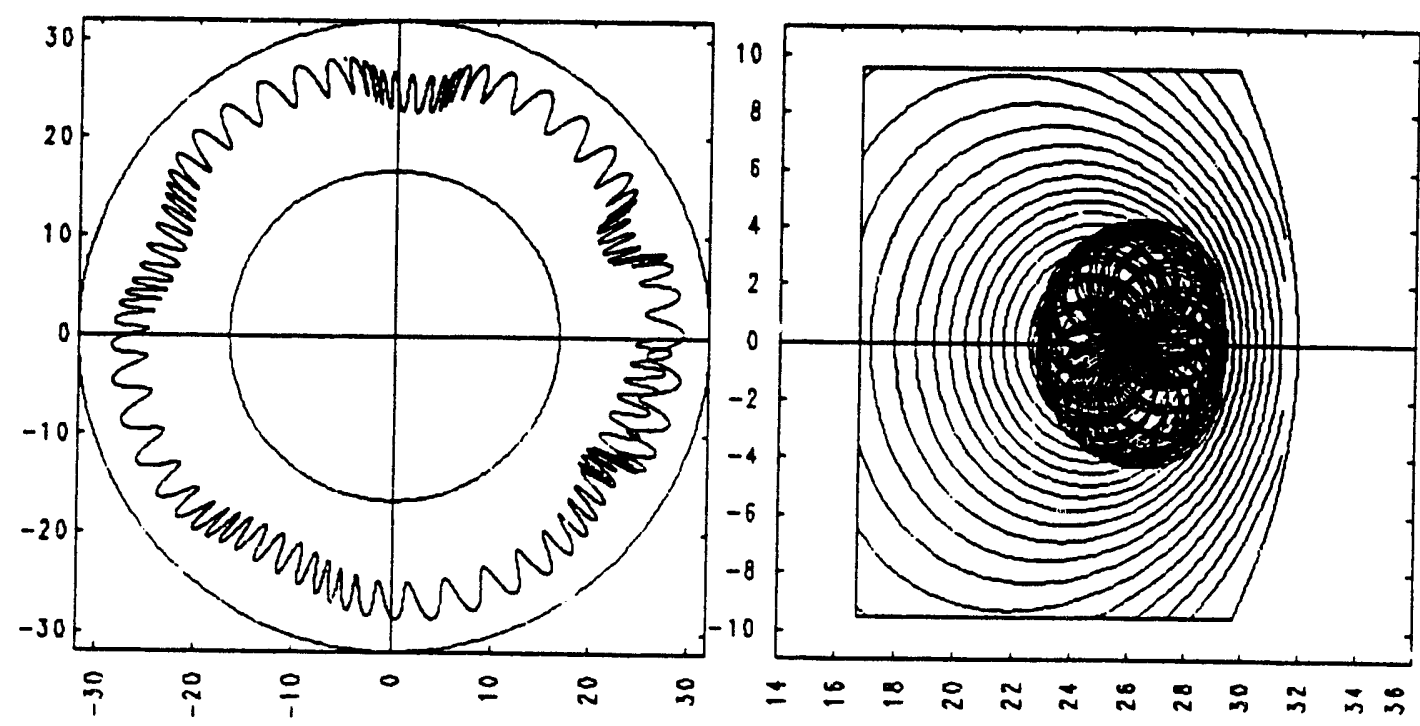

$$
\begin{aligned}
& R_{0}=26.5(\mathrm{~cm}) \\
& \theta_{i}=+14^{\circ} \\
& T=25.0(\mu \mathrm{s})
\end{aligned}
$$
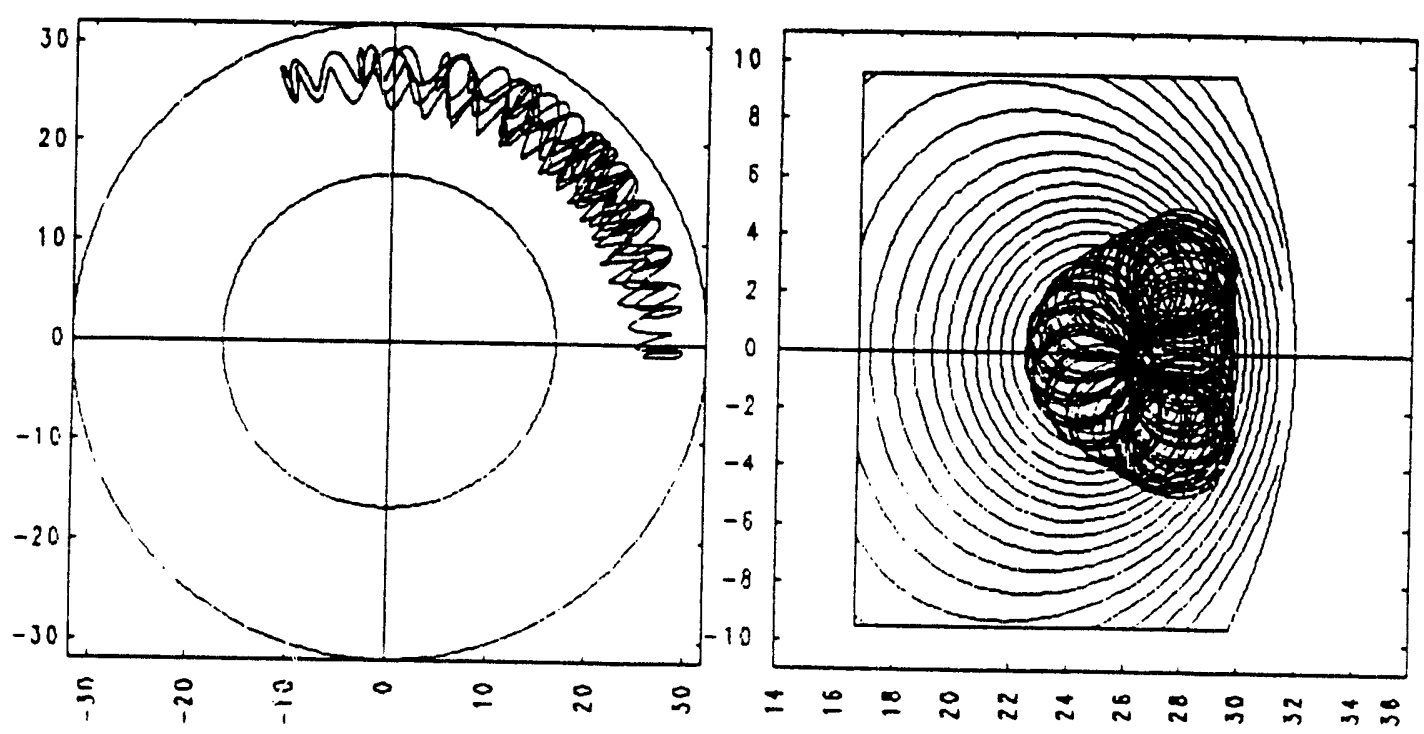

$$
\begin{aligned}
& R_{0}=28.0(\mathrm{~cm}) \\
& \theta_{i}=+14^{\circ} \\
& T=25.0(\mu \mathrm{s})
\end{aligned}
$$

Figure 2.26 The The simulations obtained at three different initial ionization locations using the magnetic field resulting from a 2-D MHD code. 


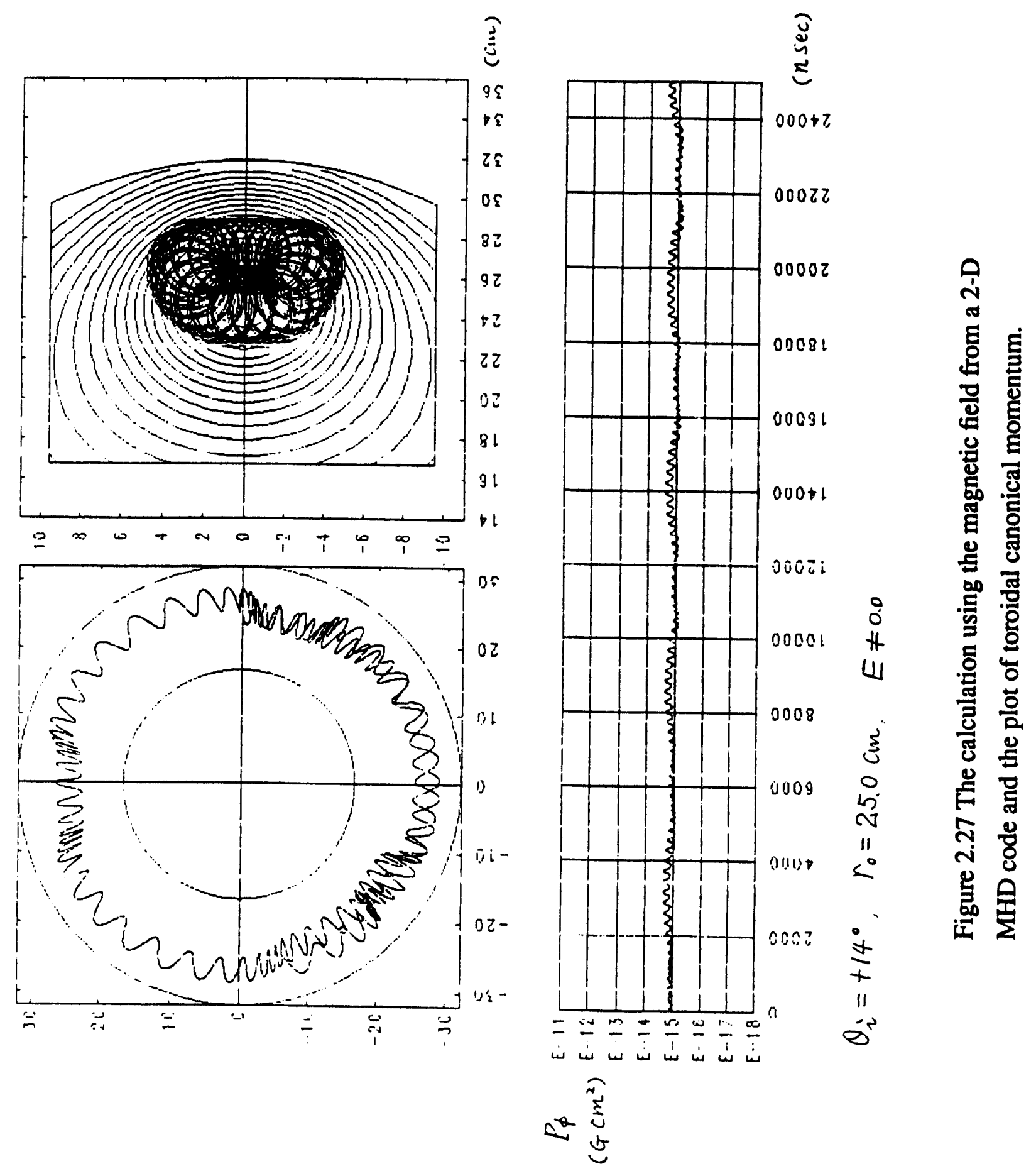



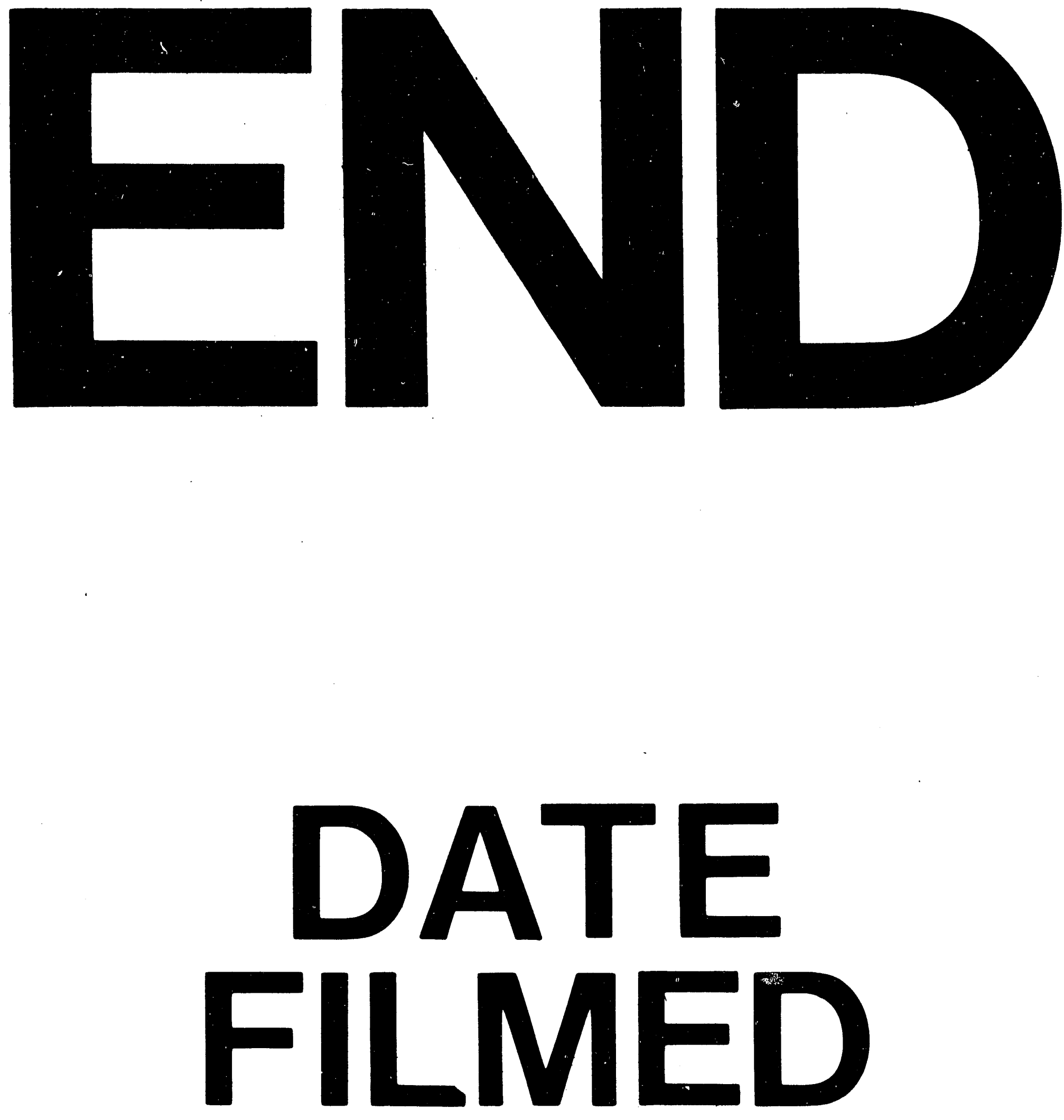

$\mathbf{A}$

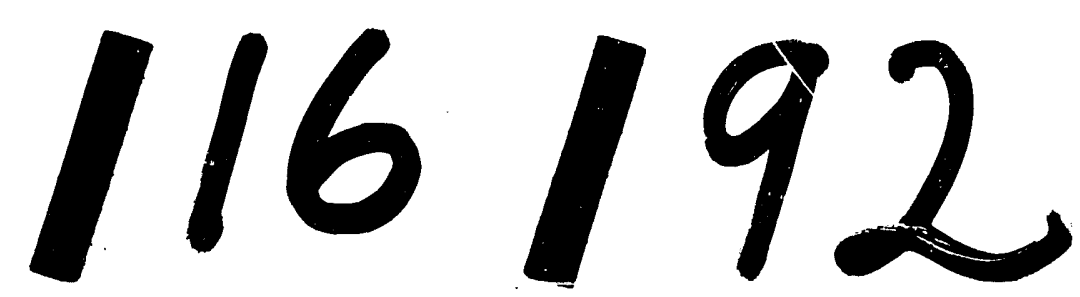


